CRYSTALLOGRAPHIC COMMUNICATIONS

ISSN 2056-9890

\section{Crystal structure of $7^{\prime}$-(4-chlorophenyl)-2"-(4- methoxyphenyl)- $7^{\prime}, 7 \mathbf{a}^{\prime}, 7^{\prime \prime}, 8^{\prime \prime}$-tetrahydro- $1^{\prime} H, 3^{\prime} H, 5^{\prime \prime} H$-dispiro[indoline-3,5'-pyrrolo[1,2-c]- thiazole- $6^{\prime}, 6^{\prime \prime}$-quinoline]-2,5"-dione and an unknown solvent}

\author{
R. Vishnupriya, ${ }^{a}$ M. Venkateshan, ${ }^{b}$ J. Suresh, ${ }^{b}$ R. V. Sumesh, ${ }^{c}$ R. Ranjith Kumar ${ }^{\mathrm{c}}$ and \\ P. L. Nilantha Lakshman ${ }^{\mathrm{d} *}$
}

Received 18 December 2018

Accepted 3 January 2019

Edited by $\mathrm{H}$. Stoeckli-Evans, University of Neuchâtel, Switzerland

Keywords: crystal structure; spiro-pyrrolidine; indoline; pyrrolothiazole; isoquinoline; hydrogen bonding; supramolecular framework; SQUEEZE.

CCDC reference: 1888600

Supporting information: this article has supporting information at journals.iucr.org/e

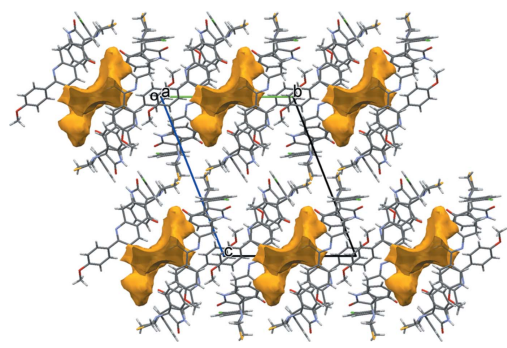

OPEN $\odot$ ACCESS a Department of Physics, The Madura College, Madurai - 625 011, India, ${ }^{\mathbf{b}}$ Department of Physics, The Madura College, Madurai 625 011, India, 'Department of Organic Chemistry, School of Chemistry, Madurai Kamaraj University, Madurai 625 021, India, and dDepartment of Food, Science and Technology, University of Ruhuna, Mapalana, Kamburupitiya 81100, Sri Lanka. *Correspondence e-mail: plakshmannilantha@ymail.com

The asymmetric unit of the title compound, $\mathrm{C}_{34} \mathrm{H}_{28} \mathrm{ClN}_{3} \mathrm{O}_{3} \mathrm{~S}$, contains two independent molecules ( $A$ and $B$ ). They differ essentially in the orientation of the 4-methoxyphenyl ring with respect to the pyridine ring of the quinoline moiety; this dihedral angle is $37.01(18)^{\circ}$ in molecule $A$ but only $7.06(17)^{\circ}$ in molecule $B$. In both molecules, the cyclohexanone ring of the isoquinoline unit has a half-chair conformation. In the pyrrolothiazole ring system, the pyrrolo ring in molecule $A$ has a twisted conformation on the $\mathrm{N}-\mathrm{C}$ fused bond and an envelope conformation in molecule $B$ with the $\mathrm{N}$ atom as the flap. The thiazole rings of both molecules have twisted conformations on the $\mathrm{N}-\mathrm{C}$ fused bond. In the crystal, the $A$ molecules are linked by pairs of $\mathrm{N}-\mathrm{H} \cdots \mathrm{O}$ hydrogen bonds, forming inversion dimers with an $R_{2}^{2}(8)$ ring motif. These dimers are linked to the $B$ molecules by an $\mathrm{N}-\mathrm{H} \cdots \mathrm{N}$ hydrogen bond and a series of $\mathrm{C}-\mathrm{H} \cdots \mathrm{O}$ hydrogen bonds, forming layers lying parallel to the (101) plane. The layers are linked by $\mathrm{C}-\mathrm{H} \cdots \pi$ interactions and offset $\pi-\pi$ interactions [intercentroid distance = 3.427 (1) $\AA$ ], forming a supramolecular framework. The contribution to the scattering from a region of highly disordered solvent molecules was removed with the SQUEEZE routine in PLATON [Spek (2015). Acta Cryst. C71, 9-18]. The solvent formula mass and unit-cell characteristics were not taken into account during refinement.

\section{Chemical context}

Pyrazolo (Siminoff et al., 1973; Zheng et al., 2006) quinoline ring systems are a privileged class of nitrogen-containing heterocycles endowed with significant biological activities. Quinoline derivatives have been reported to possess many interesting pharmacological activities and they are characteristic components of a large number of biologically active compounds. The wide spectrum of biological effects of these kind of compounds includes anti-viral (Billker et al., 1998; Roma et al., 2000; Chen et al., 2001), and antifungal (Vargas et al., 2003; Singh et al., 1996) agents. In view of their significance, the primary goal of the X-ray diffraction analysis of the title compound was to obtain detailed information on the structural conformation that may be useful in understanding the chemical reactivity of such compounds. 
<smiles></smiles>

\section{Structural commentary}

The molecular structure of the two independent molecules, $A$ and $B$, of the title compound are given in Figs. 1 and 2, respectively. The molecular overlay of inverted molecule $B$ on molecule $A$ is shown in Fig. 3. The r.m.s. deviation is $0.44 \AA$ with a maximum deviation of $1.931 \AA$ (Mercury; Macrae et al., 2008). The two molecules differ essentially in the orientation of the 4-methoxyphenyl ring (C51A-C56A, C51B-C56B) with respect to the pyridine ring of the isoquinoline moiety $(\mathrm{N} 2 A$ / $\mathrm{C} 22 A-\mathrm{C} 26 A, \mathrm{~N} 2 B / \mathrm{C} 22 B-\mathrm{C} 26 B)$. In molecule $\mathrm{A}$, the dihedral angle between the two rings is $37.01(18)^{\circ}$ compared to $7.06(17)^{\circ}$ in $B$. There is also a slight difference in the orientation of the 4-chlorophenyl ring with respect to the mean plane of the pyrrolo ring, viz. in molecule $A$ benzene ring $\mathrm{C} 11 A-\mathrm{C} 16 A$ is inclined to the mean plane of the pyrrol ring $(\mathrm{N} 1 A / \mathrm{C} 1 A-\mathrm{C} 4 A)$ by $86.12(17)^{\circ}$, while in molecule $B$ the corresponding dihedral angle is $76.92(17)^{\circ}$.

The cyclohexanone ring $[(\mathrm{C} 2 A / \mathrm{C} 21 A / \mathrm{C} 22 A / \mathrm{C} 26 A-\mathrm{C} 28 A)$ in molecule $A$ and $(\mathrm{C} 2 B / \mathrm{C} 21 B / \mathrm{C} 22 B / \mathrm{C} 26 B-\mathrm{C} 28 B)$ in $B]$ of the isoquinoline unit adopts a half-chair conformation in molecule

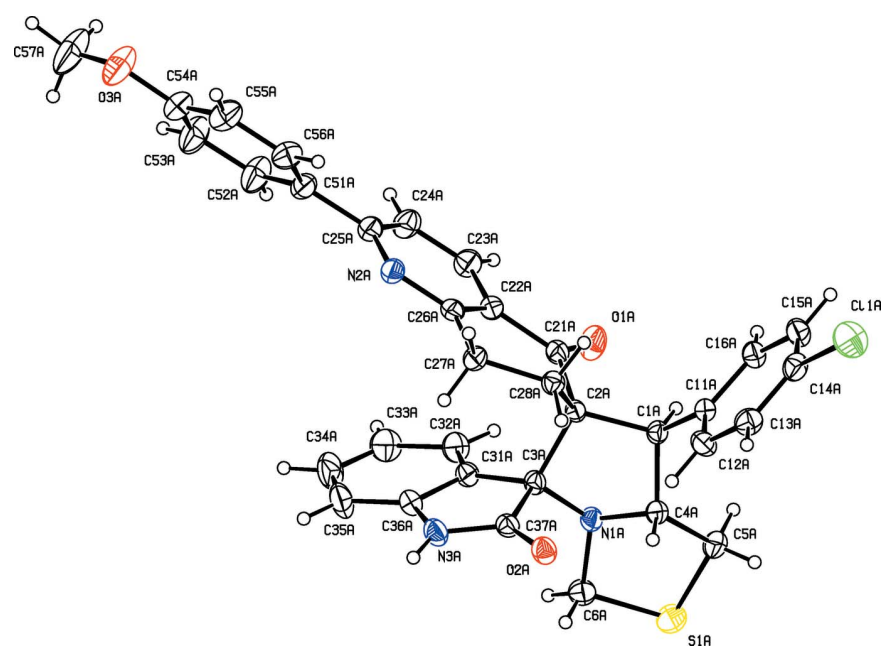

Figure 1

The molecular structure of independent molecule $A$ of the title compound, showing $30 \%$ probability displacement ellipsoids and the atom labelling.

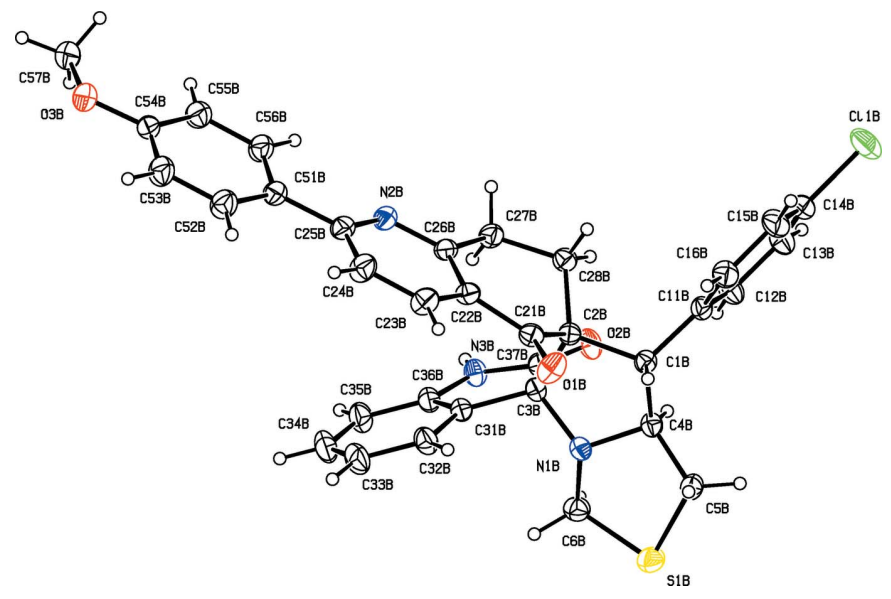

Figure 2

The molecular structure of independent molecule $B$ of the title compound, showing $30 \%$ probability displacement ellipsoids and the atom labelling.

$A$ [puckering parameters: amplitude $Q=0.441$ (3) $\AA, \theta=$ $\left.50.1(4)^{\circ}, \varphi 2=319.2(6)^{\circ}\right]$ and a distorted half-chair conformation in molecule $B$ [puckering parameters: amplitude $Q=$

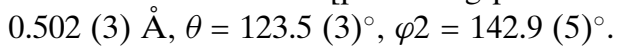

The thiazole rings have twisted conformations on bonds $\mathrm{C} 4 A-\mathrm{N} 1 A$ and $\mathrm{C} 4 B-\mathrm{N} 1 B$ for molecules $A$ and $B$, respectively. The pyrrolo ring $(\mathrm{N} 1 A / \mathrm{C} 1 A-\mathrm{C} 4 A)$ has a twisted conformation on bond $\mathrm{C} 4 A-\mathrm{N} 1 A$ in molecule $A$, while in molecule $B$ this ring $(\mathrm{N} 1 B / \mathrm{C} 1 B-\mathrm{C} 4 B)$ has an envelope conformation with atom $\mathrm{N} 1 B$ as the flap. The mean planes of the thiazole and pyrrolo rings are inclined to each other by $11.58(17)^{\circ}$ in $A$ and $12.79(18)^{\circ}$ in $B$.

In the indolin-2-one ring systems [ $(\mathrm{N} 3 A / \mathrm{C} 3 A / \mathrm{C} 31 A-\mathrm{C} 37 A)$ in $A$ and $(\mathrm{N} 3 B / \mathrm{C} 3 B / \mathrm{C} 31 B-\mathrm{C} 37 B)$ in $B]$, the benzene and pyrrolidine rings make dihedral angles of 3.65 (3) and 3.67 (3) ${ }^{\circ}$ in molecules $A$ and $B$, respectively, while the keto atoms, $\mathrm{O} 2 A$ in molecule $A$ and $\mathrm{O} 2 B$ in molecule $B$, deviate from the attached pyrrolidine rings by $0.1116(1)$ and 0.0176 (1) ^̊, respectively.

As usual for such spiro compounds, the rings involving the spiro atoms (here $\mathrm{C} 2 A / \mathrm{C} 2 B$ and $\mathrm{C} 3 A / \mathrm{C} 3 B$ ) are normal to each

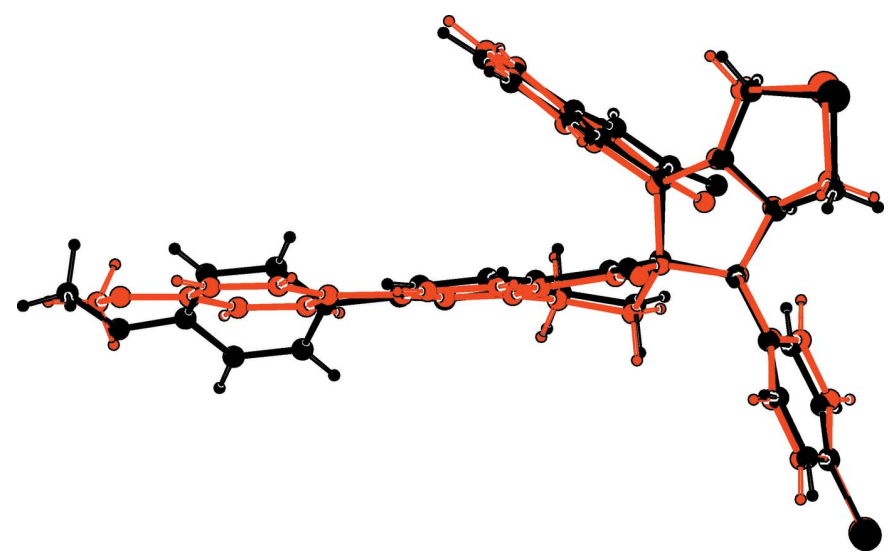

Figure 3

A view of the molecular overlay of inverted molecule $B$ (red) on molecule $A$ (black). 
Table 1

Hydrogen-bond geometry $\left(\AA,^{\circ}\right)$.

$C g 8$ and $C g 17$ are the centroids of the C51A-C56A and $\mathrm{C} 11 B-\mathrm{C} 16 B$ rings, respectively.

\begin{tabular}{lllll}
\hline$D-\mathrm{H} \cdots A$ & $D-\mathrm{H}$ & $\mathrm{H} \cdots A$ & $D \cdots A$ & $D-\mathrm{H} \cdots A$ \\
\hline $\mathrm{N} 3 A-\mathrm{H} 3 A \cdots \mathrm{O} 2 A^{\text {i }}$ & $0.83(4)$ & $2.05(4)$ & $2.874(4)$ & $172(4)$ \\
$\mathrm{N} 3 B-\mathrm{H} 3 B \cdots \mathrm{N} 2 A^{\text {ii }}$ & $0.91(4)$ & $2.24(4)$ & $3.136(4)$ & $169(3)$ \\
$\mathrm{C} 23 B-\mathrm{H} 23 B \cdots \mathrm{O} 1 A$ & 0.93 & 2.36 & $3.066(4)$ & 132 \\
$\mathrm{C} 27 A-\mathrm{H} 27 D \cdots \mathrm{O} 2 B^{\text {iii }}$ & 0.97 & 2.40 & $3.371(4)$ & 177 \\
$\mathrm{C} 28 A-\mathrm{H} 28 C \cdots \mathrm{O} 3 B^{\text {iv }}$ & 0.97 & 2.55 & $3.517(4)$ & 175 \\
$\mathrm{C} 57 B-\mathrm{H} 57 C \cdots \mathrm{O} 2 B^{\mathrm{v}}$ & 0.96 & 2.50 & $3.349(5)$ & 147 \\
$\mathrm{C} 5 A-\mathrm{H} 5 A 1 \cdots C g 17^{\mathrm{vi}}$ & 0.97 & 2.91 & $3.711(4)$ & 140 \\
$\mathrm{C} 27 B-\mathrm{H} 27 A \cdots C g 8^{\text {iv }}$ & 0.97 & 2.89 & $3.783(4)$ & 154
\end{tabular}

Symmetry codes: (i) $-x,-y+1,-z+1$; (ii) $x, y-1, z$; (iii) $x, y+1, z$; (iv) $-x+1,-y+1,-z ;(\mathrm{v})-x+1,-y,-z ;(\mathrm{vi})-x+1,-y,-z+1$.

other. In molecule $A$, the mean plane of the pyrrolo ring $(\mathrm{N} 1 A / \mathrm{C} 1 A-\mathrm{C} 4 A)$ is inclined to the mean planes of the pyrrolidine $(\mathrm{N} 3 A / \mathrm{C} 3 A / \mathrm{C} 31 A / \mathrm{C} 36 A / \mathrm{C} 37 A)$ and cyclohexanone $(\mathrm{C} 2 A / \mathrm{C} 21 A / \mathrm{C} 22 A / \mathrm{C} 26 A-\mathrm{C} 28 A)$ rings by 86.14 (18) and $84.07(12)^{\circ}$, respectively. In molecule $B$ the corresponding dihedral angles are $85.44(18)$ and $85.34(18)^{\circ}$.

\section{Supramolecular features}

In the crystal, the $A$ molecules are linked by pairs of $\mathrm{N}-$ $\mathrm{H}$... O hydrogen bonds, forming $A-A$ inversion dimers with an $R_{2}^{2}(8)$ ring motif (Fig. 4 and Table 1). These dimers are linked to the $B$ molecules by an $\mathrm{N}-\mathrm{H} \cdots \mathrm{N}$ hydrogen bond and a series of $\mathrm{C}-\mathrm{H} \cdots \mathrm{O}$ hydrogen bonds, forming layers lying parallel to the (101) plane. The layers are linked by $\mathrm{C}-\mathrm{H} \cdots \pi$ interactions (Fig. 5 and Table 1), and offset $\pi-\pi$ interactions involving the pyridine ring $(\mathrm{N} 2 A / \mathrm{C} 22 A-\mathrm{C} 26 A$; with centroid $C g 4$ ) of molecule $A$ and the 4-methoxyphenyl ring (C51B$\mathrm{C} 56 \mathrm{~B}$; with centroid $\mathrm{Cg} 19)$ of molecule $B$ : intercentroid

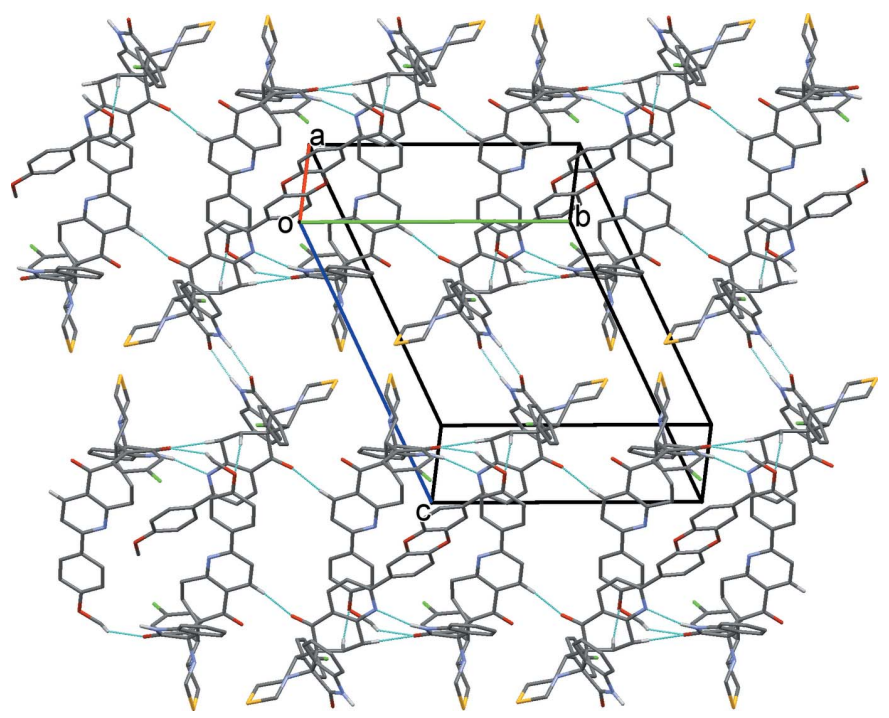

Figure 4

A view normal to plane (101) of the crystal packing of the title compound. The hydrogen bonds (see Table 1) are shown as dashed lines. $\mathrm{H}$ atoms not involved in these interactions have been omitted for clarity.

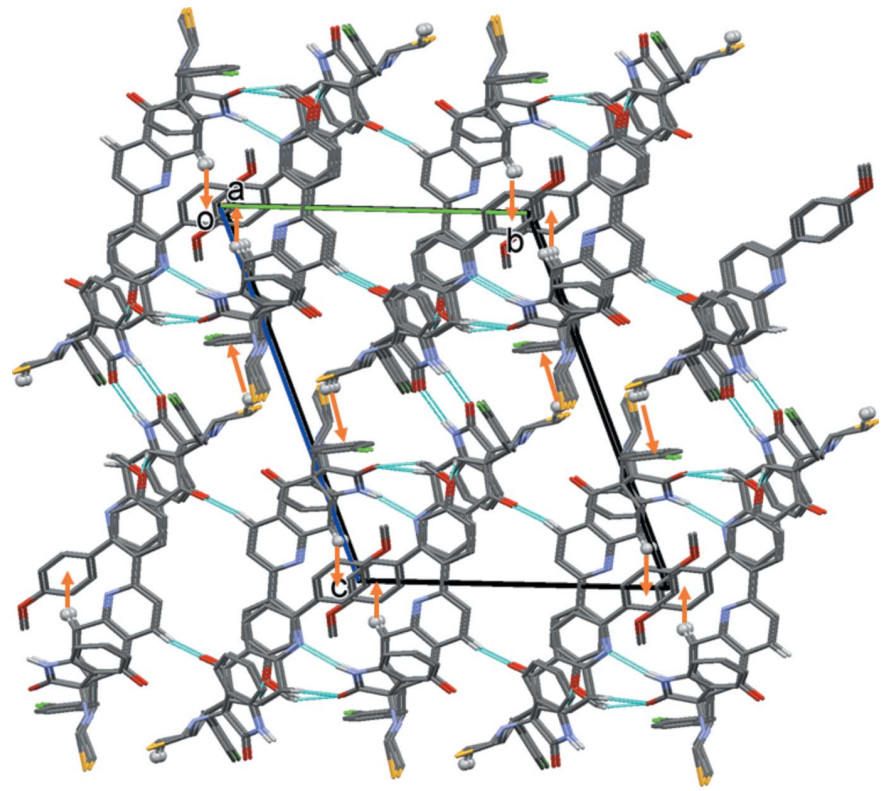

Figure 5

A view along the $a$ axis of the crystal packing of the title compound. The hydrogen bonds are shown as dashed lines and the $\mathrm{C}-\mathrm{H} \cdots \pi$ interactions (Table 1) as orange arrows. $\mathrm{H}$ atoms not involved in these interactions have been omitted for clarity.

distance $C g 4 \cdots C g 19=3.675$ (2) $\AA, \alpha=7.84(17)^{\circ}, \beta=13.3^{\circ}$, interplanar distances $=3.427(1)$ and $3.576(1) \AA$, offset $=$ $0.846 \AA$. These interactions lead to the formation of a supramolecular framework (Fig. 5).

\section{Database survey}

A search of the Cambridge Structural Database (CSD, Version 5.39, last update August 2018; Groom et al., 2016) for the central dispiro fragment, $1^{\prime}$-methyldispiro[cyclohexane/ cyclohexene-1,3'-pyrrolidine- $2^{\prime}, 3^{\prime \prime}$-indoline]-2,2' $2^{\prime \prime}$-dione, gave 16 hits (see supporting information file: CSD search.pdf). Three compounds closely resemble the title compound, viz. 4'-(4-chlorophenyl)-1'-methyl-3,4-dihydro- $1 H$-dispiro[acridine-2, $3^{\prime}$-pyrrolidine- $2^{\prime}, 3^{\prime \prime}$-indole]-1, $2^{\prime \prime}\left(1^{\prime \prime} H\right)$-dione methanol solvate (CSD refcode NAQCAL; Maheswari et al., 2012), 4'(2,4-dichlorophenyl)-10,3"-dimethyl-1" $3^{\prime \prime}$-phenyl-7", $8^{\prime \prime}$-dihydrodispiro[indole-3, $2^{\prime}$-pyrrolidine- $3^{\prime}, 6^{\prime \prime}$-pyrazolo[3,4- $b$ ]quinoline]-2,5" $\left(1 H, 1^{\prime \prime} H\right)$-dione chloroform solvate (UQIROD; Sumesh et al., 2016), and 4'-(2-chlorophenyl)-1'-methyl-2"'phenyl- $7^{\prime \prime}, 8^{\prime \prime}$-dihydro- $5^{\prime \prime} H$-dispiro[indoline-3, $2^{\prime}$-pyrrolidine$3^{\prime}, 6^{\prime \prime}$-quinoline]-2,5"'-dione (KEWKAB; Vishnupriya et al., 2018). Three other compounds contain a pyrrolothiazole moiety; they are the dispiro[cyclohexane-pyrrolothiazoleindole]-dione derivatives RAGMUK, RAGNAR and RAHBIO (Lotfy et al., 2017). In all six compounds, the mean plane of the pyrrolidine ring was found to be almost perpendicular to the mean plane of the indoline ring system and the mean plane of the cyclohexanone ring, similar to the situation in the title compound. 
Table 2

Experimental details.

\begin{tabular}{|c|c|}
\hline \multicolumn{2}{|l|}{ Crystal data } \\
\hline Chemical formula & $\mathrm{C}_{34} \mathrm{H}_{28} \mathrm{ClN}_{3} \mathrm{O}_{3} \mathrm{~S}$ \\
\hline$M_{\mathrm{r}}$ & 594.10 \\
\hline Crystal system, space group & Triclinic, $P \overline{1}$ \\
\hline Temperature $(\mathrm{K})$ & 293 \\
\hline$a, b, c(\AA)$ & $\begin{array}{l}11.8222(7), 14.7535(9), \\
19.5055(12)\end{array}$ \\
\hline$\alpha, \beta, \gamma\left({ }^{\circ}\right)$ & $68.396(3), 78.555(3), 87.302(3)$ \\
\hline$V\left(\AA^{3}\right)$ & $3099.0(3)$ \\
\hline$Z$ & 4 \\
\hline Radiation type & Мо $K \alpha$ \\
\hline$\mu\left(\mathrm{mm}^{-1}\right)$ & 0.23 \\
\hline Crystal size (mm) & $0.21 \times 0.2 \times 0.18$ \\
\hline \multicolumn{2}{|l|}{ Data collection } \\
\hline Diffractometer & Bruker Kappa APEXII \\
\hline Absorption correction & $\begin{array}{l}\text { Multi-scan (SADABS; Bruker, } \\
\text { 2009) }\end{array}$ \\
\hline$T_{\min }, T_{\max }$ & $0.967,0.974$ \\
\hline $\begin{array}{l}\text { No. of measured, independent and } \\
\text { observed }[I>2 \sigma(I)] \text { reflections }\end{array}$ & $78936,12885,7510$ \\
\hline$R_{\text {int }}$ & 0.072 \\
\hline$(\sin \theta / \lambda)_{\max }\left(\AA^{-1}\right)$ & 0.630 \\
\hline \multicolumn{2}{|l|}{ Refinement } \\
\hline$R\left[F^{2}>2 \sigma\left(F^{2}\right)\right], w R\left(F^{2}\right), S$ & $0.058,0.171,1.01$ \\
\hline No. of reflections & 12885 \\
\hline No. of parameters & 765 \\
\hline $\mathrm{H}$-atom treatment & $\begin{array}{l}\mathrm{H} \text { atoms treated by a mixture of } \\
\text { independent and constrained } \\
\text { refinement }\end{array}$ \\
\hline$\Delta \rho_{\max }, \Delta \rho_{\min }\left(\mathrm{e} \AA^{-3}\right)$ & $0.40,-0.32$ \\
\hline
\end{tabular}

Computer programs: APEX2 and SAINT (Bruker, 2009), SHELXS2013 (Sheldrick, 2008), SHELXL2014/6 (Sheldrick, 2015), ORTEP-3 for Windows (Farrugia, 2012), Mercury (Macrae et al., 2008), PLATON (Spek, 2009) and publCIF (Westrip, 2010).

\section{Synthesis and crystallization}

A mixture of isatin $(1.1 \mathrm{mmol})$ and thiazolidine-4-carboxylic acid $(1.1 \mathrm{mmol})$ was taken in $10 \mathrm{ml}$ of acetonitrile in a $50 \mathrm{ml}$ round bottom flask and heated to reflux for $2 \mathrm{~h}$. Then $(E)-6-(4-$ chlorobenzylidene)-2-(4-methoxyphenyl)-7,8-dihydroquinolin-5(6H)-one (1 mmol) was added and the reaction mixture was allowed to reflux for a further $14 \mathrm{~h}$. After completion of the reaction, as evident from TLC, the solvent was removed under reduced pressure and the residue washed with ice-cold water $(50 \mathrm{ml})$. The crude product was purified by column chromatography using 90:10 ( $v / v)$ petroleum ether-ethyl acetate mixtures to obtain the pure product. The product was dissolved in ethyl acetate and poured into a beaker, covered with perforated film and kept undisturbed. The solvent was allowed to evaporate slowly, yielding colourless block-like crystals after a period of seven days (m.p. 458 K; yield 80\%).

\section{Refinement}

Crystal data, data collection and structure refinement details are summarized in Table 2. The $\mathrm{NH} \mathrm{H}$ atoms were located in a difference-Fourier map and freely refined. The C-bound $\mathrm{H}$ atoms were placed at calculated positions and allowed to ride on their carrier atoms: $\mathrm{C}-\mathrm{H}=0.93-0.98 \AA$ with $U_{\text {iso }}=$ $1.5 U_{\text {eq }}(\mathrm{C}$-methyl $)$ and $1.2 U_{\text {eq }}(\mathrm{C})$ for other $\mathrm{H}$ atoms. The

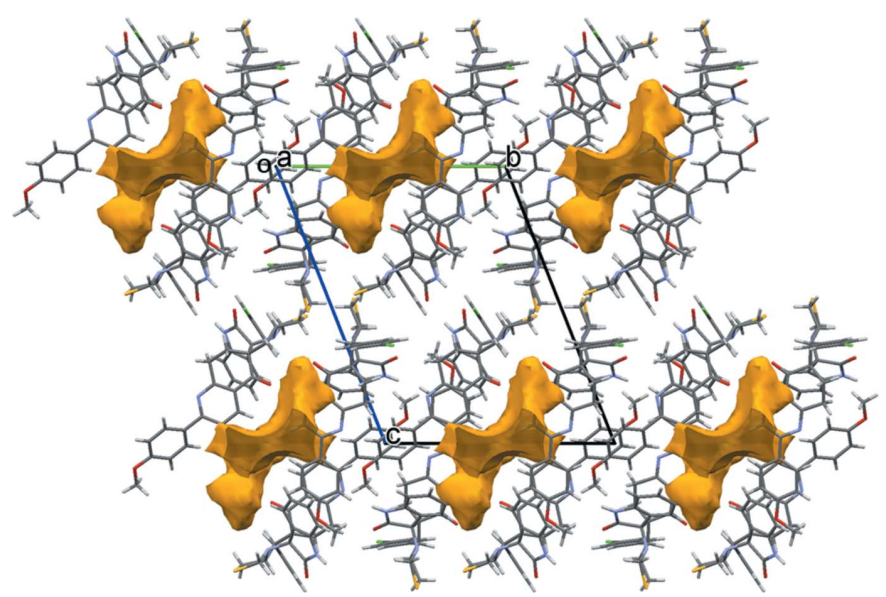

Figure 6

A view of the solvent-accessible surface (yellow/brown) in the crystal of the title compound.

residual electron density was difficult to model and therefore, the SQUEEZE routine in PLATON (Spek, 2015) was used to remove the contribution of the electron density in the solvent region from the intensity data and the solvent-free model was employed for the final refinement. The solvent formula mass and unit-cell characteristics were not taken into account during refinement. The cavity of volume ca $418 \AA^{3}$ (ca $14 \%$ of the unit-cell volume) contains approximately 100 electrons (see Fig. 6).

\section{Acknowledgements}

JS and RV thank the management of Madura College for their encouragement.

\section{Funding information}

JS thanks the UGC for funds under project No. F MRP-7018/ 16(SERO/UGC).

\section{References}

Billker, O., Lindo, V., Panico, M., Etienne, A. E., Paxton, T., Dell, A., Rogers, M., Sinden, R. E. \& Morris, H. R. (1998). Nature, 392, 289 292.

Bruker (2009). APEX2, SAINT and SADABS. Bruker AXS Inc., Madison, Wisconsin, USA.

Chen, Y.-L., Fang, K.-C., Sheu, J.-Y., Hsu, S.-L. \& Tzeng, C.-C. (2001). J. Med. Chem. 44, 2374-2377.

Farrugia, L. J. (2012). J. Appl. Cryst. 45, 849-854.

Groom, C. R., Bruno, I. J., Lightfoot, M. P. \& Ward, S. C. (2016). Acta Cryst. B72, 171-179.

Lotfy, G., Said, M. M., El Ashry, E. S. H., El Tamany, E. S. H., AlDhfyan, A., Abdel Aziz, Y. M. \& Barakat, A. (2017). Bioorg. Med. Chem. 25, 1514-1523.

Macrae, C. F., Bruno, I. J., Chisholm, J. A., Edgington, P. R., McCabe, P., Pidcock, E., Rodriguez-Monge, L., Taylor, R., van de Streek, J. \& Wood, P. A. (2008). J. Appl. Cryst. 41, 466-470.

Maheswari, S. U., Perumal, S. \& Almansour, A. I. (2012). Tetrahedron Lett. 53, 349-353.

Roma, G., Di Braccio, M., Grossi, G., Mattioli, F. \& Ghia, H. (2000). Eur. J. Med. Chem. 35, 1021-1035.

Sheldrick, G. M. (2008). Acta Cryst. A64, 112-122.

Sheldrick, G. M. (2015). Acta Cryst. C71, 3-8. 
Siminoff, P., Bernard, A. M., Hursky, V. S. \& Price, K. E. (1973). Antimicrob. Agents Chemother. 3, 742-743.

Singh, M., Singh, M. P. \& Ablordeppey, S. Y. (1996). Drug Dev. Ind. Pharm. 22, 377-381.

Spek, A. L. (2009). Acta Cryst. D65, 148-155.

Spek, A. L. (2015). Acta Cryst. C71, 9-18.

Sumesh, R. V., Muthu, M., Almansour, A. I., Kumar, R. S., Arumugam, N., Athimoolam, S., Jeya Yasmi Prabha, E. A. \& Kumar, R. R. (2016). ACS Comb. Sci. 18, 262-270.
Vargas, L. Y., Castelli, M. V., Kouznetsov, V. V., Urbina, J. M., López, S. N., Sortino, M., Enriz, R. D., Ribas, J. C. \& Zacchino, S. (2003). Bioorg. Med. Chem. 11, 1531-1550.

Vishnupriya, R., Selva Meenatchi, C., Suresh, J., Sumesh, R. V., Kumar, R. R. \& Lakshman, P. L. N. (2018). Acta Cryst. E74, 660663.

Westrip, S. P. (2010). J. Appl. Cryst. 43, 920-925.

Zheng, A., Zhang, W. \& Pan, J. (2006). Synth. Commun. 36, 15491556. 


\section{supporting information}

Acta Cryst. (2019). E75, 189-193 [https://doi.org/10.1107/S2056989019000112]

Crystal structure of $7^{\prime}-(4-c h l o r o p h e n y l)-2^{\prime \prime}-(4-m e t h o x y p h e n y l)-7^{\prime}, 7 a^{\prime}, 7^{\prime \prime}, 8^{\prime \prime}-$ tetrahydro-1' $H, 3^{\prime} H, 5^{\prime \prime} H$-dispiro[indoline-3,5'-pyrrolo[1,2-c] thiazole-6', 6'"quinoline]-2,5"-dione and an unknown solvent

R. Vishnupriya, M. Venkateshan, J. Suresh, R. V. Sumesh, R. Ranjith Kumar and P. L. Nilantha

\section{Lakshman}

Computing details

Data collection: APEX2 (Bruker, 2009); cell refinement: SAINT (Bruker, 2009); data reduction: SAINT (Bruker, 2009); program(s) used to solve structure: SHELXS2013 (Sheldrick, 2008); program(s) used to refine structure: SHELXL2014/6 (Sheldrick, 2015); molecular graphics: ORTEP-3 for Windows (Farrugia, 2012) and Mercury (Macrae et al., 2008); software used to prepare material for publication: SHELXL2014/6 (Sheldrick, 2015), PLATON (Spek, 2009) and publCIF (Westrip, 2010).

7'-(4-Chlorophenyl)-2"'-(4-methoxyphenyl)-7',7a',7", 8' -tetrahydro-1' H, 3' H,5' $H$-dispiro[indoline-3, 5'pyrrolo[1,2-c] thiazole-6',6"'-quinoline]-2,5"-dione

Crystal data

$\mathrm{C}_{34} \mathrm{H}_{28} \mathrm{ClN}_{3} \mathrm{O}_{3} \mathrm{~S}$

$M_{r}=594.10$

Triclinic, $P \overline{1}$

$a=11.8222(7) \AA$

$b=14.7535(9) \AA$

$c=19.5055(12) \AA$

$\alpha=68.396(3)^{\circ}$

$\beta=78.555(3)^{\circ}$

$\gamma=87.302(3)^{\circ}$

$V=3099.0(3) \AA^{3}$

Data collection

Bruker Kappa APEXII

diffractometer

Radiation source: fine-focus sealed tube

Detector resolution: 0 pixels $\mathrm{mm}^{-1}$

$\omega$ and $\varphi$ scans

Absorption correction: multi-scan

(SADABS; Bruker, 2009)

$T_{\min }=0.967, T_{\max }=0.974$
$Z=4$

$F(000)=1240$

$D_{\mathrm{x}}=1.273 \mathrm{Mg} \mathrm{m}^{-3}$

Mo $K \alpha$ radiation, $\lambda=0.71073 \AA$

Cell parameters from 12885 reflections

$\theta=2.2-26.6^{\circ}$

$\mu=0.23 \mathrm{~mm}^{-1}$

$T=293 \mathrm{~K}$

Block, colourless

$0.21 \times 0.2 \times 0.18 \mathrm{~mm}$

78936 measured reflections 12885 independent reflections 7510 reflections with $I>2 \sigma(I)$

$R_{\text {int }}=0.072$

$\theta_{\text {max }}=26.6^{\circ}, \theta_{\min }=2.2^{\circ}$

$h=-14 \rightarrow 14$

$k=-18 \rightarrow 18$

$l=-24 \rightarrow 24$ 


\section{Refinement}

Refinement on $F^{2}$

Least-squares matrix: full

$R\left[F^{2}>2 \sigma\left(F^{2}\right)\right]=0.058$

$w R\left(F^{2}\right)=0.171$

$S=1.01$

12885 reflections

765 parameters

0 restraints

Primary atom site location: structure-invariant direct methods
Secondary atom site location: difference Fourier map

Hydrogen site location: difference Fourier map

$\mathrm{H}$ atoms treated by a mixture of independent and constrained refinement

$w=1 /\left[\sigma^{2}\left(F_{\mathrm{o}}^{2}\right)+(0.067 P)^{2}+2.7018 P\right]$ where $P=\left(F_{\mathrm{o}}^{2}+2 F_{\mathrm{c}}^{2}\right) / 3$

$(\Delta / \sigma)_{\max }=0.001$

$\Delta \rho_{\max }=0.40 \mathrm{e} \AA^{-3}$

$\Delta \rho_{\min }=-0.32$ e $\AA^{-3}$

Special details

Geometry. All esds (except the esd in the dihedral angle between two 1.s. planes) are estimated using the full covariance matrix. The cell esds are taken into account individually in the estimation of esds in distances, angles and torsion angles; correlations between esds in cell parameters are only used when they are defined by crystal symmetry. An approximate (isotropic) treatment of cell esds is used for estimating esds involving l.s. planes.

Fractional atomic coordinates and isotropic or equivalent isotropic displacement parameters $\left(\AA^{2}\right)$

\begin{tabular}{|c|c|c|c|c|}
\hline & $x$ & $y$ & $z$ & $U_{\text {iso }} * / U_{\text {eq }}$ \\
\hline $\mathrm{C} 1 \mathrm{~B}$ & $0.5260(2)$ & -0.0249 & $0.35951(16)$ & $0.0323(7)$ \\
\hline H1B & 0.5318 & 0.0323 & 0.3729 & $0.039 *$ \\
\hline $\mathrm{C} 1 \mathrm{~A}$ & $0.3327(2)$ & $0.3432(2)$ & $0.36470(16)$ & $0.0308(6)$ \\
\hline $\mathrm{H} 1 \mathrm{~A}$ & 0.3600 & 0.3148 & 0.3264 & $0.037 *$ \\
\hline $\mathrm{C} 2 \mathrm{~B}$ & $0.4873(3)$ & $0.0134(2)$ & $0.28085(16)$ & $0.0321(7)$ \\
\hline $\mathrm{C} 2 \mathrm{~A}$ & $0.2713(2)$ & $0.44096(19)$ & $0.32533(15)$ & $0.0284(6)$ \\
\hline $\mathrm{C} 3 \mathrm{~B}$ & $0.3566(2)$ & $-0.0264(2)$ & $0.30098(16)$ & $0.0312(6)$ \\
\hline C3A & $0.1371(2)$ & $0.4122(2)$ & $0.35761(16)$ & $0.0320(7)$ \\
\hline C4B & $0.4242(2)$ & $-0.0873(2)$ & $0.41544(16)$ & $0.0341(7)$ \\
\hline $\mathrm{H} 4 \mathrm{~B}$ & 0.4308 & -0.1549 & 0.4179 & $0.041^{*}$ \\
\hline $\mathrm{C} 4 \mathrm{~A}$ & $0.2363(2)$ & 0.2758 & $0.42160(16)$ & $0.0326(7)$ \\
\hline $\mathrm{H} 4 \mathrm{~A}$ & 0.2214 & 0.2894 & 0.4681 & $0.039 *$ \\
\hline C5B & 0.3932 & -0.0848 & $0.49356(17)$ & $0.0428(8)$ \\
\hline H5A & 0.4114 & -0.0211 & 0.4932 & $0.051 *$ \\
\hline H5B & 0.4345 & -0.1337 & 0.5275 & $0.051 *$ \\
\hline C5A & $0.2420(3)$ & $0.1669(2)$ & 0.43995 (19) & $0.0426(8)$ \\
\hline H5A1 & 0.2781 & 0.1356 & 0.4831 & $0.051 *$ \\
\hline $\mathrm{H} 5 \mathrm{~A} 2$ & 0.2854 & 0.1527 & 0.3975 & $0.051 *$ \\
\hline C6B & 0.2225 & $-0.0925(2)$ & $0.42665(17)$ & $0.0426(8)$ \\
\hline H6A & 0.2145 & -0.1544 & 0.4210 & $0.051 *$ \\
\hline H6B & 0.1558 & -0.0538 & 0.4139 & $0.051 *$ \\
\hline C6A & $0.0382(3)$ & $0.2480(2)$ & $0.4275(2)$ & $0.0464(8)$ \\
\hline H6A1 & -0.0193 & 0.2527 & 0.3972 & $0.056^{*}$ \\
\hline H6A2 & 0.0046 & 0.2678 & 0.4692 & $0.056^{*}$ \\
\hline $\mathrm{C} 11 \mathrm{~B}$ & 0.6425 & $-0.0712(2)$ & $0.36006(16)$ & $0.0345(7)$ \\
\hline C11A & $0.4363(2)$ & $0.3534(2)$ & $0.39620(16)$ & $0.0314(6)$ \\
\hline C12B & $0.6578(3)$ & $-0.1687(2)$ & 0.37073 (19) & $0.0439(8)$ \\
\hline H12B & 0.5939 & -0.2084 & 0.3787 & $0.053 *$ \\
\hline
\end{tabular}




\begin{tabular}{|c|c|c|c|c|}
\hline $\mathrm{C} 12 \mathrm{~A}$ & $0.4272(3)$ & $0.3740(2)$ & $0.46068(18)$ & $0.0421(8)$ \\
\hline $\mathrm{H} 12 \mathrm{~A}$ & 0.3546 & 0.3823 & 0.4862 & $0.050 *$ \\
\hline $\mathrm{C} 13 \mathrm{~B}$ & $0.7665(3)$ & $-0.2084(2)$ & 0.36978 (19) & $0.0474(8)$ \\
\hline H13B & 0.7751 & -0.2743 & 0.3776 & $0.057^{*}$ \\
\hline $\mathrm{C} 13 \mathrm{~A}$ & $0.5241(3)$ & $0.3824(2)$ & $0.48810(18)$ & $0.0443(8)$ \\
\hline $\mathrm{H} 13 \mathrm{~A}$ & 0.5167 & 0.3964 & 0.5315 & $0.053^{*}$ \\
\hline $\mathrm{C} 14 \mathrm{~B}$ & $0.8603(3)$ & $-0.1508(3)$ & $0.35735(18)$ & $0.0471(8)$ \\
\hline $\mathrm{C} 14 \mathrm{~A}$ & $0.6301(3)$ & $0.3699(2)$ & $0.45095(19)$ & $0.0422(8)$ \\
\hline $\mathrm{C} 15 \mathrm{~B}$ & $0.8488(3)$ & $-0.0547(3)$ & $0.3474(2)$ & $0.0525(9)$ \\
\hline H15B & 0.9134 & -0.0158 & 0.3396 & $0.063^{*}$ \\
\hline $\mathrm{C} 15 \mathrm{~A}$ & $0.6430(3)$ & $0.3497(2)$ & $0.3871(2)$ & $0.0461(8)$ \\
\hline $\mathrm{H} 15 \mathrm{~A}$ & 0.7162 & 0.3422 & 0.3618 & $0.055^{*}$ \\
\hline $\mathrm{C} 16 \mathrm{~B}$ & $0.7397(3)$ & $-0.0152(2)$ & $0.34917(18)$ & $0.0450(8)$ \\
\hline H16B & 0.7318 & 0.0502 & 0.3429 & $0.054^{*}$ \\
\hline C16A & $0.5461(3)$ & $0.3405(2)$ & $0.36039(18)$ & $0.0387(7)$ \\
\hline H16A & 0.5546 & 0.3252 & 0.3175 & $0.046^{*}$ \\
\hline $\mathrm{C} 21 \mathrm{~B}$ & $0.4888(3)$ & $0.1254(2)$ & $0.25042(17)$ & $0.0359(7)$ \\
\hline $\mathrm{C} 21 \mathrm{~A}$ & $0.2926(3)$ & $0.4648(2)$ & $0.24032(16)$ & $0.0345(7)$ \\
\hline $\mathrm{C} 22 \mathrm{~B}$ & $0.4879(3)$ & $0.1759(2)$ & $0.16965(16)$ & $0.0354(7)$ \\
\hline $\mathrm{C} 22 \mathrm{~A}$ & $0.2763(3)$ & $0.5667(2)$ & $0.18981(16)$ & $0.0345(7)$ \\
\hline $\mathrm{C} 23 \mathrm{~B}$ & $0.4699(3)$ & $0.2756(2)$ & $0.14136(18)$ & $0.0465(8)$ \\
\hline $\mathrm{H} 23 \mathrm{~B}$ & 0.4663 & 0.3118 & 0.1719 & $0.056^{*}$ \\
\hline $\mathrm{C} 23 \mathrm{~A}$ & $0.2758(3)$ & $0.5862(2)$ & $0.11482(18)$ & $0.0459(8)$ \\
\hline $\mathrm{H} 23 \mathrm{~A}$ & 0.2877 & 0.5364 & 0.0959 & $0.055^{*}$ \\
\hline $\mathrm{C} 24 \mathrm{~B}$ & $0.4577(3)$ & $0.3198(2)$ & $0.06846(18)$ & $0.0491(9)$ \\
\hline $\mathrm{H} 24 \mathrm{~B}$ & 0.4470 & 0.3867 & 0.0489 & $0.059^{*}$ \\
\hline $\mathrm{C} 24 \mathrm{~A}$ & $0.2579(3)$ & $0.6787(3)$ & 0.06877 (19) & $0.0488(9)$ \\
\hline $\mathrm{H} 24 \mathrm{~A}$ & 0.2563 & 0.6924 & 0.0185 & $0.059^{*}$ \\
\hline $\mathrm{C} 25 \mathrm{~B}$ & $0.4613(3)$ & $0.2653(2)$ & $0.02361(17)$ & $0.0407(8)$ \\
\hline $\mathrm{C} 25 \mathrm{~A}$ & $0.2422(3)$ & $0.7517(2)$ & $0.09796(18)$ & $0.0409(8)$ \\
\hline $\mathrm{C} 26 \mathrm{~B}$ & $0.4963(3)$ & $0.1263(2)$ & $0.12049(16)$ & $0.0338(7)$ \\
\hline C26A & $0.2602(2)$ & $0.6436(2)$ & $0.21523(16)$ & $0.0340(7)$ \\
\hline C27B & $0.5222(3)$ & $0.0205(2)$ & $0.14588(17)$ & $0.0393(7)$ \\
\hline $\mathrm{H} 27 \mathrm{~A}$ & 0.5808 & 0.0091 & 0.1076 & $0.047^{*}$ \\
\hline $\mathrm{H} 27 \mathrm{~B}$ & 0.4530 & -0.0165 & 0.1515 & $0.047 *$ \\
\hline C27A & $0.2574(3)$ & $0.6260(2)$ & $0.29575(16)$ & $0.0361(7)$ \\
\hline $\mathrm{H} 27 \mathrm{C}$ & 0.1779 & 0.6257 & 0.3209 & $0.043^{*}$ \\
\hline $\mathrm{H} 27 \mathrm{D}$ & 0.2984 & 0.6794 & 0.2989 & $0.043^{*}$ \\
\hline $\mathrm{C} 28 \mathrm{~B}$ & $0.5642(3)$ & $-0.0159(2)$ & $0.21994(16)$ & $0.0367(7)$ \\
\hline $\mathrm{H} 28 \mathrm{~A}$ & 0.6419 & 0.0098 & 0.2114 & $0.044 *$ \\
\hline $\mathrm{H} 28 \mathrm{~B}$ & 0.5675 & -0.0865 & 0.2378 & $0.044^{*}$ \\
\hline $\mathrm{C} 28 \mathrm{~A}$ & $0.3113(3)$ & $0.5301(2)$ & $0.33682(16)$ & $0.0329(7)$ \\
\hline $\mathrm{H} 28 \mathrm{C}$ & 0.3945 & 0.5375 & 0.3204 & $0.040^{*}$ \\
\hline $\mathrm{H} 28 \mathrm{D}$ & 0.2941 & 0.5181 & 0.3902 & $0.040^{*}$ \\
\hline C31B & $0.2667(3)$ & $0.0329(2)$ & $0.26030(16)$ & $0.0343(7)$ \\
\hline C31A & $0.0542(3)$ & $0.4546(2)$ & $0.30558(17)$ & $0.0353(7)$ \\
\hline C32B & $0.2235(3)$ & $0.1228(2)$ & $0.25610(19)$ & $0.0447(8)$ \\
\hline H32B & 0.2546 & 0.1598 & 0.2778 & $0.054^{*}$ \\
\hline
\end{tabular}




\begin{tabular}{|c|c|c|c|c|}
\hline $\mathrm{C} 32 \mathrm{~A}$ & $0.0418(3)$ & $0.4413(2)$ & $0.24147(19)$ & $0.0463(8)$ \\
\hline $\mathrm{H} 32 \mathrm{~A}$ & 0.0889 & 0.3991 & 0.2239 & $0.056^{*}$ \\
\hline C33B & $0.1324(3)$ & $0.1571(3)$ & $0.2188(2)$ & $0.0576(10)$ \\
\hline H33B & 0.1031 & 0.2181 & 0.2147 & $0.069 *$ \\
\hline C33A & $-0.0430(3)$ & $0.4924(3)$ & $0.2033(2)$ & $0.0586(10)$ \\
\hline $\mathrm{H} 33 \mathrm{~A}$ & -0.0522 & 0.4848 & 0.1594 & $0.070^{*}$ \\
\hline C34B & $0.0855(3)$ & $0.1012(3)$ & $0.1878(2)$ & $0.0591(10)$ \\
\hline H34B & 0.0253 & 0.1256 & 0.1625 & $0.071 *$ \\
\hline $\mathrm{C} 34 \mathrm{~A}$ & $-0.1127(3)$ & $0.5535(3)$ & $0.2300(2)$ & $0.0685(12)$ \\
\hline H34A & -0.1675 & 0.5885 & 0.2031 & $0.082 *$ \\
\hline $\mathrm{C} 35 \mathrm{~B}$ & $0.1254(3)$ & $0.0101(3)$ & $0.1932(2)$ & $0.0501(9)$ \\
\hline H35B & 0.0927 & -0.0279 & 0.1728 & $0.060^{*}$ \\
\hline $\mathrm{C} 35 \mathrm{~A}$ & $-0.1038(3)$ & $0.5645(3)$ & $0.2956(2)$ & $0.0620(11)$ \\
\hline $\mathrm{H} 35 \mathrm{~A}$ & -0.1526 & 0.6052 & 0.3139 & $0.074 *$ \\
\hline $\mathrm{C} 36 \mathrm{~B}$ & $0.2163(3)$ & $-0.0223(2)$ & $0.23029(17)$ & $0.0370(7)$ \\
\hline $\mathrm{C} 36 \mathrm{~A}$ & $-0.0204(3)$ & $0.5134(2)$ & $0.33343(18)$ & $0.0396(7)$ \\
\hline C37B & $0.3501(3)$ & $-0.1224(2)$ & $0.28654(16)$ & $0.0342(7)$ \\
\hline C37A & $0.0894(3)$ & $0.4501(2)$ & $0.42181(17)$ & $0.0330(7)$ \\
\hline $\mathrm{C} 51 \mathrm{~B}$ & $0.4430(3)$ & $0.3075(2)$ & $-0.05451(17)$ & $0.0391(7)$ \\
\hline C51A & $0.2227(3)$ & $0.8532(2)$ & $0.04837(18)$ & $0.0434(8)$ \\
\hline $\mathrm{C} 52 \mathrm{~B}$ & $0.4088(3)$ & $0.4027(3)$ & $-0.0863(2)$ & $0.0526(9)$ \\
\hline H52B & 0.3966 & 0.4420 & -0.0578 & $0.063 *$ \\
\hline $\mathrm{C} 52 \mathrm{~A}$ & $0.1596(4)$ & $0.8708(3)$ & $-0.0074(2)$ & $0.0645(11)$ \\
\hline $\mathrm{H} 52 \mathrm{~A}$ & 0.1286 & 0.8181 & -0.0135 & $0.077^{*}$ \\
\hline C53B & $0.3926(3)$ & $0.4398(3)$ & $-0.1591(2)$ & $0.0526(9)$ \\
\hline H53B & 0.3707 & 0.5041 & -0.1797 & $0.063^{*}$ \\
\hline $\mathrm{C} 53 \mathrm{~A}$ & 0.1407 (4) & $0.9640(3)$ & $-0.0545(2)$ & $0.0702(12)$ \\
\hline H53A & 0.0978 & 0.9734 & -0.0916 & $0.084 *$ \\
\hline $\mathrm{C} 54 \mathrm{~B}$ & $0.4085(3)$ & $0.3825(2)$ & $-0.20156(17)$ & $0.0407(8)$ \\
\hline C54A & $0.1851(3)$ & $1.0421(3)$ & $-0.0465(2)$ & $0.0586(10)$ \\
\hline C55B & $0.4435(3)$ & $0.2881(2)$ & $-0.17192(19)$ & $0.0473(8)$ \\
\hline H55B & 0.4561 & 0.2493 & -0.2009 & $0.057^{*}$ \\
\hline C55A & $0.2505(4)$ & $1.0263(3)$ & $0.0077(2)$ & $0.0597(10)$ \\
\hline H55A & 0.2830 & 1.0791 & 0.0127 & $0.072 *$ \\
\hline $\mathrm{C} 56 \mathrm{~B}$ & $0.4595(3)$ & $0.2517(2)$ & $-0.09857(18)$ & $0.0468(8)$ \\
\hline H56B & 0.4821 & 0.1876 & -0.0783 & $0.056^{*}$ \\
\hline $\mathrm{C} 56 \mathrm{~A}$ & $0.2681(3)$ & $0.9333(3)$ & $0.05454(19)$ & $0.0511(9)$ \\
\hline H56A & 0.3117 & 0.9243 & 0.0912 & $0.061^{*}$ \\
\hline N1B & $0.3275(2)$ & $-0.04168(17)$ & $0.38060(13)$ & $0.0329(6)$ \\
\hline N1A & $0.1396(2)$ & $0.30634(17)$ & $0.38312(13)$ & $0.0336(6)$ \\
\hline $\mathrm{N} 2 \mathrm{~B}$ & $0.4825(2)$ & $0.16914(18)$ & 0.04989 (14) & $0.0394(6)$ \\
\hline $\mathrm{N} 2 \mathrm{~A}$ & $0.2435(2)$ & $0.73521(18)$ & $0.17025(14)$ & $0.0389(6)$ \\
\hline N3B & $0.2667(2)$ & $-0.11384(19)$ & $0.24572(15)$ & $0.0378(6)$ \\
\hline N3A & $0.0019(2)$ & $0.5097(2)$ & $0.40164(16)$ & $0.0423(7)$ \\
\hline O1B & $0.4912(2)$ & $0.17151(15)$ & $0.28999(12)$ & $0.0506(6)$ \\
\hline O1A & $0.3175(2)$ & $0.40314(17)$ & $0.21365(12)$ & $0.0573(7)$ \\
\hline $\mathrm{O} 2 \mathrm{~B}$ & $0.40684(19)$ & $-0.19446(15)$ & $0.30953(13)$ & $0.0441(5)$ \\
\hline $\mathrm{O} 2 \mathrm{~A}$ & $0.12263(18)$ & $0.42887(15)$ & $0.48137(12)$ & $0.0412(5)$ \\
\hline
\end{tabular}




$\begin{array}{lllll}\text { O3B } & 0.3894(2) & 0.42566(17) & -0.27308(12) & 0.0518(6) \\ \text { O3A } & 0.1723(3) & 1.13645(19) & -0.09003(17) & 0.0847(10) \\ \text { S1B } & 0.23841(8) & -0.11082(7) & 0.52141(5) & 0.0504(2) \\ \text { S1A } & 0.09202(9) & 0.12520(6) & 0.46069(6) & 0.0564(3) \\ \text { C11B } & 0.99560(8) & -0.20181(9) & 0.35523(7) & 0.0754(3) \\ \text { C11A } & 0.75107(9) & 0.38243(8) & 0.48536(6) & 0.0711(3) \\ \text { C57B } & 0.3971(3) & 0.3675(3) & -0.3168(2) & 0.0545(9) \\ \text { H57A } & 0.3819 & 0.4063 & -0.3656 & 0.082^{*} \\ \text { H57C } & 0.4732 & 0.3421 & -0.3221 & 0.082^{*} \\ \text { H57B } & 0.3413 & 0.3146 & -0.2927 & 0.082^{*} \\ \text { C57A } & 0.1111(5) & 1.1555(4) & -0.1488(3) & 0.112(2) \\ \text { H57D } & 0.1086 & 1.2246 & -0.1749 & 0.168^{*} \\ \text { H57E } & 0.1492 & 1.1259 & -0.1833 & 0.168^{*} \\ \text { H57F } & 0.0338 & 1.1288 & -0.1283 & 0.168^{*} \\ \text { H3B } & 0.251(3) & -0.161(3) & 0.229(2) & 0.063(12)^{*} \\ \text { H3A } & -0.037(4) & 0.532(3) & 0.432(2) & 0.074(14)^{*}\end{array}$

Atomic displacement parameters $\left(\AA^{2}\right)$

\begin{tabular}{|c|c|c|c|c|c|c|}
\hline & $U^{11}$ & $U^{22}$ & $U^{33}$ & $U^{12}$ & $U^{13}$ & $U^{23}$ \\
\hline $\mathrm{C} 1 \mathrm{~B}$ & $0.0321(16)$ & $0.0331(15)$ & $0.0321(16)$ & $-0.0013(13)$ & $-0.0072(13)$ & $-0.0117(13)$ \\
\hline $\mathrm{C} 1 \mathrm{~A}$ & $0.0332(16)$ & $0.0284(15)$ & $0.0301(15)$ & $0.0057(12)$ & $-0.0062(13)$ & $-0.0105(12)$ \\
\hline $\mathrm{C} 2 \mathrm{~B}$ & $0.0333(16)$ & $0.0323(15)$ & $0.0322(16)$ & $0.0036(13)$ & $-0.0071(13)$ & $-0.0136(13)$ \\
\hline $\mathrm{C} 2 \mathrm{~A}$ & $0.0255(15)$ & $0.0279(14)$ & $0.0319(15)$ & $0.0032(12)$ & $-0.0055(12)$ & $-0.0115(12)$ \\
\hline C3B & $0.0300(16)$ & $0.0305(15)$ & $0.0361(16)$ & $0.0050(12)$ & $-0.0112(13)$ & $-0.0138(13)$ \\
\hline $\mathrm{C} 3 \mathrm{~A}$ & $0.0325(16)$ & $0.0281(15)$ & $0.0367(16)$ & $0.0046(12)$ & $-0.0059(13)$ & $-0.0141(13)$ \\
\hline C4B & $0.0326(16)$ & $0.0326(16)$ & $0.0364(17)$ & $0.0001(13)$ & $-0.0100(13)$ & $-0.0100(13)$ \\
\hline $\mathrm{C} 4 \mathrm{~A}$ & $0.0331(16)$ & $0.0301(15)$ & $0.0340(16)$ & $0.0033(13)$ & $-0.0053(13)$ & $-0.0119(13)$ \\
\hline C5B & $0.0398(19)$ & $0.052(2)$ & $0.0340(17)$ & $-0.0041(15)$ & $-0.0109(15)$ & $-0.0099(15)$ \\
\hline $\mathrm{C} 5 \mathrm{~A}$ & $0.048(2)$ & $0.0299(16)$ & $0.0466(19)$ & $0.0050(14)$ & $-0.0085(16)$ & $-0.0112(14)$ \\
\hline C6B & $0.0390(19)$ & $0.0427(18)$ & $0.0428(19)$ & $-0.0013(15)$ & $-0.0031(15)$ & $-0.0140(15)$ \\
\hline C6A & $0.0373(19)$ & $0.0365(17)$ & $0.060(2)$ & $-0.0059(14)$ & $-0.0059(16)$ & $-0.0130(16)$ \\
\hline C11B & $0.0303(16)$ & $0.0394(17)$ & $0.0325(16)$ & $-0.0012(13)$ & $-0.0086(13)$ & $-0.0100(13)$ \\
\hline $\mathrm{C} 11 \mathrm{~A}$ & $0.0284(16)$ & $0.0301(15)$ & $0.0329(16)$ & $0.0039(12)$ & -0.0060 & $-0.0086(13)$ \\
\hline C12B & $0.0315(17)$ & $0.0454(19)$ & $0.057(2)$ & $-0.0010(14)$ & $-0.0123(15)$ & $-0.0185(16)$ \\
\hline $\mathrm{C} 12 \mathrm{~A}$ & $0.0315(17)$ & $0.052(2)$ & $0.0418(19)$ & $0.0042(15)$ & $-0.0043(14)$ & $-0.0177(16)$ \\
\hline C13B & $0.041(2)$ & $0.0433(19)$ & $0.057(2)$ & $0.0096(16)$ & $-0.0143(17)$ & $-0.0164(17)$ \\
\hline $\mathrm{C} 13 \mathrm{~A}$ & $0.048(2)$ & $0.0467(19)$ & $0.0387(18)$ & $-0.0006(16)$ & $-0.0112(16)$ & $-0.0143(15)$ \\
\hline C14B & $0.0302(18)$ & $0.062(2)$ & $0.0420(19)$ & $0.0042(16)$ & $-0.0066(15)$ & $-0.0117(17)$ \\
\hline $\mathrm{C} 14 \mathrm{~A}$ & $0.0399(19)$ & $0.0363(17)$ & $0.050(2)$ & $-0.0017(14)$ & $-0.0193(16)$ & $-0.0099(15)$ \\
\hline C15B & $0.0294(18)$ & $0.063(2)$ & $0.059(2)$ & $-0.0086(16)$ & $-0.0085(16)$ & $-0.0143(19)$ \\
\hline $\mathrm{C} 15 \mathrm{~A}$ & $0.0334(18)$ & $0.0461(19)$ & $0.057(2)$ & $0.0070(15)$ & $-0.0095(16)$ & $-0.0177(17)$ \\
\hline C16B & $0.0388(19)$ & $0.0455(19)$ & $0.047(2)$ & $-0.0065(15)$ & $-0.0089(16)$ & $-0.0112(16)$ \\
\hline $\mathrm{C} 16 \mathrm{~A}$ & $0.0363(18)$ & $0.0392(17)$ & $0.0418(18)$ & $0.0067(14)$ & $-0.0083(15)$ & $-0.0165(14)$ \\
\hline C21B & $0.0370(17)$ & $0.0340(16)$ & $0.0394(18)$ & $0.0028(13)$ & $-0.0059(14)$ & $-0.0176(14)$ \\
\hline $\mathrm{C} 21 \mathrm{~A}$ & $0.0331(17)$ & $0.0358(16)$ & $0.0345(17)$ & $0.0053(13)$ & $-0.0041(13)$ & $-0.0147(14)$ \\
\hline C22B & $0.0373(17)$ & $0.0338(16)$ & $0.0323(16)$ & $0.0001(13)$ & $-0.0014(13)$ & $-0.0116(13)$ \\
\hline $\mathrm{C} 22 \mathrm{~A}$ & $0.0332(17)$ & $0.0362(16)$ & $0.0327(16)$ & $0.0032(13)$ & $-0.0060(13)$ & $-0.0113(13)$ \\
\hline
\end{tabular}




\begin{tabular}{|c|c|c|c|c|c|c|}
\hline C23B & $0.062(2)$ & $0.0357(17)$ & $0.0421(19)$ & $0.0043(16)$ & $-0.0073(17)$ & $-0.0164(15)$ \\
\hline $\mathrm{C} 23 \mathrm{~A}$ & $0.053(2)$ & $0.0440(19)$ & $0.0404(19)$ & $0.0042(16)$ & $-0.0071(16)$ & $-0.0170(16)$ \\
\hline $\mathrm{C} 24 \mathrm{~B}$ & $0.068(2)$ & $0.0322(17)$ & $0.041(2)$ & $0.0078(16)$ & $-0.0086(17)$ & $-0.0084(15)$ \\
\hline $\mathrm{C} 24 \mathrm{~A}$ & $0.056(2)$ & $0.053(2)$ & $0.0357(18)$ & $0.0036(17)$ & $-0.0158(17)$ & $-0.0101(16)$ \\
\hline $\mathrm{C} 25 \mathrm{~B}$ & $0.0413(19)$ & $0.0405(18)$ & $0.0342(17)$ & $0.0030(15)$ & $-0.0029(14)$ & $-0.0092(14)$ \\
\hline $\mathrm{C} 25 \mathrm{~A}$ & $0.0369(18)$ & $0.0405(18)$ & $0.0396(19)$ & $0.0002(14)$ & $-0.0086(15)$ & $-0.0072(15)$ \\
\hline $\mathrm{C} 26 \mathrm{~B}$ & $0.0331(16)$ & $0.0355(16)$ & $0.0313(16)$ & $0.0015(13)$ & $-0.0026(13)$ & $-0.0125(13)$ \\
\hline $\mathrm{C} 26 \mathrm{~A}$ & $0.0258(15)$ & $0.0376(17)$ & $0.0354(17)$ & $-0.0021(13)$ & $-0.0037(13)$ & $-0.0104(14)$ \\
\hline $\mathrm{C} 27 \mathrm{~B}$ & 0.0460 (19) & $0.0384(17)$ & $0.0358(17)$ & $0.0068(15)$ & $-0.0077(15)$ & $-0.0170(14)$ \\
\hline $\mathrm{C} 27 \mathrm{~A}$ & $0.0384(18)$ & $0.0273(15)$ & $0.0410(18)$ & $0.0000(13)$ & $-0.0068(14)$ & $-0.0110(13)$ \\
\hline $\mathrm{C} 28 \mathrm{~B}$ & $0.0383(18)$ & $0.0347(16)$ & $0.0373(17)$ & $0.0037(14)$ & $-0.0066(14)$ & $-0.0142(14)$ \\
\hline $\mathrm{C} 28 \mathrm{~A}$ & $0.0322(16)$ & $0.0338(16)$ & $0.0335(16)$ & $0.0012(13)$ & $-0.0082(13)$ & $-0.0123(13)$ \\
\hline $\mathrm{C} 31 \mathrm{~B}$ & $0.0326(17)$ & $0.0379(17)$ & $0.0339(16)$ & $0.0051(13)$ & $-0.0093(13)$ & $-0.0139(13)$ \\
\hline C31A & $0.0300(16)$ & $0.0375(17)$ & $0.0383(17)$ & $0.0006(13)$ & $-0.0101(14)$ & $-0.0119(14)$ \\
\hline C32B & $0.043(2)$ & $0.0434(19)$ & $0.052(2)$ & $0.0129(15)$ & $-0.0165(16)$ & $-0.0202(16)$ \\
\hline $\mathrm{C} 32 \mathrm{~A}$ & $0.046(2)$ & $0.048(2)$ & $0.050(2)$ & $0.0043(16)$ & $-0.0157(17)$ & $-0.0213(16)$ \\
\hline C33B & $0.058(2)$ & $0.051(2)$ & $0.065(2)$ & $0.0263(19)$ & $-0.023(2)$ & -0.0205 (19) \\
\hline C33A & $0.053(2)$ & $0.073(3)$ & $0.054(2)$ & $-0.001(2)$ & -0.0235 (19) & $-0.022(2)$ \\
\hline C34B & $0.048(2)$ & $0.067(3)$ & $0.065(3)$ & $0.0194(19)$ & $-0.0293(19)$ & $-0.021(2)$ \\
\hline $\mathrm{C} 34 \mathrm{~A}$ & $0.049(2)$ & $0.089(3)$ & $0.068(3)$ & $0.024(2)$ & $-0.031(2)$ & $-0.023(2)$ \\
\hline C35B & $0.044(2)$ & $0.058(2)$ & $0.055(2)$ & $0.0074(17)$ & $-0.0232(17)$ & $-0.0214(18)$ \\
\hline C35A & $0.044(2)$ & $0.079(3)$ & $0.067(3)$ & $0.029(2)$ & -0.0198 (19) & $-0.030(2)$ \\
\hline C36B & $0.0349(17)$ & $0.0376(17)$ & $0.0379(17)$ & $0.0036(14)$ & $-0.0114(14)$ & $-0.0113(14)$ \\
\hline $\mathrm{C} 36 \mathrm{~A}$ & $0.0311(17)$ & $0.0419(18)$ & $0.0435(19)$ & $0.0067(14)$ & $-0.0054(14)$ & $-0.0146(15)$ \\
\hline C37B & $0.0335(17)$ & $0.0344(16)$ & $0.0352(17)$ & $0.0041(13)$ & $-0.0099(14)$ & $-0.0120(13)$ \\
\hline C37A & $0.0306(16)$ & $0.0316(15)$ & $0.0371(17)$ & $0.0005(13)$ & -0.0039 & $-0.0142(13)$ \\
\hline $\mathrm{C} 51 \mathrm{~B}$ & $0.0400(18)$ & $0.0371(17)$ & $0.0349(17)$ & $0.0017(14)$ & $-0.0053(14)$ & $-0.0085(14)$ \\
\hline C51A & $0.045(2)$ & $0.0366(17)$ & $0.0416(19)$ & $-0.0016(15)$ & $-0.0101(16)$ & $-0.0054(15)$ \\
\hline $\mathrm{C} 52 \mathrm{~B}$ & $0.065(2)$ & $0.046(2)$ & $0.046(2)$ & $0.0096(18)$ & $-0.0132(18)$ & $-0.0164(17)$ \\
\hline $\mathrm{C} 52 \mathrm{~A}$ & $0.076(3)$ & $0.046(2)$ & $0.071(3)$ & $-0.001(2)$ & $-0.040(2)$ & $-0.0081(19)$ \\
\hline C53B & $0.062(2)$ & $0.0424(19)$ & $0.048(2)$ & $0.0092(17)$ & $-0.0147(18)$ & $-0.0086(17)$ \\
\hline C53A & $0.082(3)$ & $0.056(2)$ & $0.069(3)$ & $0.004(2)$ & $-0.042(2)$ & $-0.005(2)$ \\
\hline C54B & $0.0330(17)$ & $0.0470(19)$ & $0.0338(17)$ & $0.0016(14)$ & $-0.0051(14)$ & $-0.0060(15)$ \\
\hline $\mathrm{C} 54 \mathrm{~A}$ & $0.063(3)$ & $0.047(2)$ & $0.049(2)$ & $0.0032(19)$ & $-0.0100(19)$ & $0.0007(18)$ \\
\hline C55B & $0.052(2)$ & $0.0453(19)$ & $0.044(2)$ & $0.0076(16)$ & $-0.0097(16)$ & $-0.0155(16)$ \\
\hline C55A & $0.078(3)$ & $0.044(2)$ & $0.053(2)$ & $-0.0039(19)$ & $-0.011(2)$ & $-0.0130(18)$ \\
\hline C56B & $0.055(2)$ & $0.0403(18)$ & $0.0395(19)$ & $0.0086(16)$ & $-0.0094(16)$ & $-0.0087(15)$ \\
\hline C56A & $0.058(2)$ & $0.052(2)$ & $0.0384(19)$ & $-0.0020(18)$ & $-0.0086(17)$ & $-0.0116(16)$ \\
\hline N1B & $0.0287(13)$ & $0.0336(13)$ & $0.0365(14)$ & $0.0015(11)$ & $-0.0087(11)$ & $-0.0121(11)$ \\
\hline N1A & $0.0303(14)$ & $0.0285(13)$ & $0.0409(14)$ & $0.0016(10)$ & $-0.0077(11)$ & $-0.0112(11)$ \\
\hline $\mathrm{N} 2 \mathrm{~B}$ & $0.0431(16)$ & $0.0391(15)$ & $0.0322(14)$ & $0.0039(12)$ & $-0.0059(12)$ & $-0.0098(12)$ \\
\hline N2A & $0.0345(15)$ & $0.0352(14)$ & $0.0419(16)$ & -0.0001 & $-0.0102(12)$ & $-0.0066(12)$ \\
\hline N3B & $0.0388(15)$ & $0.0352(14)$ & $0.0447(16)$ & $0.0011(12)$ & $-0.0164(13)$ & $-0.0166(12)$ \\
\hline N3A & $0.0369(16)$ & $0.0492(17)$ & $0.0423(17)$ & $0.0120(13)$ & $-0.0048(13)$ & $-0.0215(14)$ \\
\hline O1B & $0.0823(18)$ & $0.0331(12)$ & $0.0411(13)$ & $0.0022(12)$ & $-0.0141(12)$ & $-0.0178(10)$ \\
\hline $\mathrm{O} 1 \mathrm{~A}$ & $0.0852(19)$ & $0.0486(14)$ & $0.0425(14)$ & $0.0285(13)$ & $-0.0128(13)$ & $-0.0248(12)$ \\
\hline $\mathrm{O} 2 \mathrm{~B}$ & $0.0461(14)$ & $0.0334(12)$ & $0.0592(15)$ & $0.0087(10)$ & $-0.0226(11)$ & $-0.0192(11)$ \\
\hline $\mathrm{O} 2 \mathrm{~A}$ & $0.0410(13)$ & $0.0474(13)$ & $0.0384(13)$ & $0.0095(10)$ & $-0.0080(10)$ & $-0.0201(10)$ \\
\hline
\end{tabular}




\begin{tabular}{lllllll}
\hline & & & & & \\
O3B & $0.0545(15)$ & $0.0529(14)$ & $0.0413(13)$ & $0.0095(12)$ & $-0.0129(11)$ & $-0.0086(11)$ \\
O3A & $0.111(3)$ & $0.0476(16)$ & $0.081(2)$ & $0.0039(16)$ & $-0.0381(19)$ & $0.0030(15)$ \\
S1B & $0.0416(5)$ & $0.0637(6)$ & $0.0402(5)$ & $-0.0072(4)$ & $-0.0027(4)$ & $-0.0140(4)$ \\
S1A & $0.0539(6)$ & $0.0348(5)$ & $0.0721(7)$ & $-0.0071(4)$ & $-0.0061(5)$ & $-0.0121(4)$ \\
C11B & $0.0347(5)$ & $0.0922(8)$ & $0.0890(8)$ & $0.0150(5)$ & $-0.0113(5)$ & $-0.0234(6)$ \\
C11A & $0.0504(6)$ & $0.0867(7)$ & $0.0837(7)$ & $-0.0068(5)$ & $-0.0301(5)$ & $-0.0301(6)$ \\
C57B & $0.051(2)$ & $0.070(2)$ & $0.046(2)$ & $0.0097(19)$ & $-0.0162(17)$ & $-0.0227(19)$ \\
C57A & $0.151(6)$ & $0.072(3)$ & $0.091(4)$ & $0.016(3)$ & $-0.061(4)$ & $0.013(3)$ \\
\hline
\end{tabular}

Geometric parameters $\left(\AA,{ }^{\circ}\right)$

\begin{tabular}{|c|c|c|c|}
\hline $\mathrm{C} 1 \mathrm{~B}-\mathrm{C} 11 \mathrm{~B}$ & $1.509(4)$ & $\mathrm{C} 24 \mathrm{~A}-\mathrm{H} 24 \mathrm{~A}$ & 0.9300 \\
\hline $\mathrm{C} 1 \mathrm{~B}-\mathrm{C} 4 \mathrm{~B}$ & $1.526(4)$ & $\mathrm{C} 25 \mathrm{~B}-\mathrm{N} 2 \mathrm{~B}$ & $1.349(4)$ \\
\hline $\mathrm{C} 1 \mathrm{~B}-\mathrm{C} 2 \mathrm{~B}$ & $1.580(4)$ & $\mathrm{C} 25 \mathrm{~B}-\mathrm{C} 51 \mathrm{~B}$ & $1.475(4)$ \\
\hline $\mathrm{C} 1 \mathrm{~B}-\mathrm{H} 1 \mathrm{~B}$ & 0.9800 & $\mathrm{C} 25 \mathrm{~A}-\mathrm{N} 2 \mathrm{~A}$ & $1.343(4)$ \\
\hline $\mathrm{C} 1 \mathrm{~A}-\mathrm{C} 11 \mathrm{~A}$ & $1.512(4)$ & $\mathrm{C} 25 \mathrm{~A}-\mathrm{C} 51 \mathrm{~A}$ & $1.490(4)$ \\
\hline $\mathrm{C} 1 \mathrm{~A}-\mathrm{C} 4 \mathrm{~A}$ & $1.521(4)$ & $\mathrm{C} 26 \mathrm{~B}-\mathrm{N} 2 \mathrm{~B}$ & $1.327(4)$ \\
\hline $\mathrm{C} 1 \mathrm{~A}-\mathrm{C} 2 \mathrm{~A}$ & $1.582(4)$ & $\mathrm{C} 26 \mathrm{~B}-\mathrm{C} 27 \mathrm{~B}$ & $1.491(4)$ \\
\hline $\mathrm{C} 1 \mathrm{~A}-\mathrm{H} 1 \mathrm{~A}$ & 0.9800 & $\mathrm{C} 26 \mathrm{~A}-\mathrm{N} 2 \mathrm{~A}$ & $1.344(4)$ \\
\hline $\mathrm{C} 2 \mathrm{~B}-\mathrm{C} 28 \mathrm{~B}$ & $1.529(4)$ & $\mathrm{C} 26 \mathrm{~A}-\mathrm{C} 27 \mathrm{~A}$ & 1.489 (4) \\
\hline $\mathrm{C} 2 \mathrm{~B}-\mathrm{C} 21 \mathrm{~B}$ & $1.535(4)$ & $\mathrm{C} 27 \mathrm{~B}-\mathrm{C} 28 \mathrm{~B}$ & $1.520(4)$ \\
\hline $\mathrm{C} 2 \mathrm{~B}-\mathrm{C} 3 \mathrm{~B}$ & $1.601(4)$ & $\mathrm{C} 27 \mathrm{~B}-\mathrm{H} 27 \mathrm{~A}$ & 0.9700 \\
\hline $\mathrm{C} 2 \mathrm{~A}-\mathrm{C} 28 \mathrm{~A}$ & $1.523(4)$ & $\mathrm{C} 27 \mathrm{~B}-\mathrm{H} 27 \mathrm{~B}$ & 0.9700 \\
\hline $\mathrm{C} 2 \mathrm{~A}-\mathrm{C} 21 \mathrm{~A}$ & $1.533(4)$ & $\mathrm{C} 27 \mathrm{~A}-\mathrm{C} 28 \mathrm{~A}$ & $1.527(4)$ \\
\hline $\mathrm{C} 2 \mathrm{~A}-\mathrm{C} 3 \mathrm{~A}$ & $1.601(4)$ & $\mathrm{C} 27 \mathrm{~A}-\mathrm{H} 27 \mathrm{C}$ & 0.9700 \\
\hline $\mathrm{C} 3 \mathrm{~B}-\mathrm{N} 1 \mathrm{~B}$ & $1.455(4)$ & $\mathrm{C} 27 \mathrm{~A}-\mathrm{H} 27 \mathrm{D}$ & 0.9700 \\
\hline $\mathrm{C} 3 \mathrm{~B}-\mathrm{C} 31 \mathrm{~B}$ & $1.503(4)$ & $\mathrm{C} 28 \mathrm{~B}-\mathrm{H} 28 \mathrm{~A}$ & 0.9700 \\
\hline $\mathrm{C} 3 \mathrm{~B}-\mathrm{C} 37 \mathrm{~B}$ & $1.550(4)$ & $\mathrm{C} 28 \mathrm{~B}-\mathrm{H} 28 \mathrm{~B}$ & 0.9700 \\
\hline $\mathrm{C} 3 \mathrm{~A}-\mathrm{N} 1 \mathrm{~A}$ & $1.455(3)$ & $\mathrm{C} 28 \mathrm{~A}-\mathrm{H} 28 \mathrm{C}$ & 0.9700 \\
\hline $\mathrm{C} 3 \mathrm{~A}-\mathrm{C} 31 \mathrm{~A}$ & $1.501(4)$ & $\mathrm{C} 28 \mathrm{~A}-\mathrm{H} 28 \mathrm{D}$ & 0.9700 \\
\hline $\mathrm{C} 3 \mathrm{~A}-\mathrm{C} 37 \mathrm{~A}$ & $1.551(4)$ & $\mathrm{C} 31 \mathrm{~B}-\mathrm{C} 36 \mathrm{~B}$ & $1.377(4)$ \\
\hline $\mathrm{C} 4 \mathrm{~B}-\mathrm{N} 1 \mathrm{~B}$ & $1.457(4)$ & $\mathrm{C} 31 \mathrm{~B}-\mathrm{C} 32 \mathrm{~B}$ & $1.379(4)$ \\
\hline $\mathrm{C} 4 \mathrm{~B}-\mathrm{C} 5 \mathrm{~B}$ & $1.509(4)$ & $\mathrm{C} 31 \mathrm{~A}-\mathrm{C} 32 \mathrm{~A}$ & $1.371(4)$ \\
\hline $\mathrm{C} 4 \mathrm{~B}-\mathrm{H} 4 \mathrm{~B}$ & 0.9800 & $\mathrm{C} 31 \mathrm{~A}-\mathrm{C} 36 \mathrm{~A}$ & $1.381(4)$ \\
\hline $\mathrm{C} 4 \mathrm{~A}-\mathrm{N} 1 \mathrm{~A}$ & $1.454(4)$ & $\mathrm{C} 32 \mathrm{~B}-\mathrm{C} 33 \mathrm{~B}$ & $1.391(4)$ \\
\hline $\mathrm{C} 4 \mathrm{~A}-\mathrm{C} 5 \mathrm{~A}$ & $1.513(4)$ & $\mathrm{C} 32 \mathrm{~B}-\mathrm{H} 32 \mathrm{~B}$ & 0.9300 \\
\hline $\mathrm{C} 4 \mathrm{~A}-\mathrm{H} 4 \mathrm{~A}$ & 0.9800 & $\mathrm{C} 32 \mathrm{~A}-\mathrm{C} 33 \mathrm{~A}$ & $1.392(5)$ \\
\hline $\mathrm{C} 5 \mathrm{~B}-\mathrm{S} 1 \mathrm{~B}$ & $1.819(3)$ & $\mathrm{C} 32 \mathrm{~A}-\mathrm{H} 32 \mathrm{~A}$ & 0.9300 \\
\hline $\mathrm{C} 5 \mathrm{~B}-\mathrm{H} 5 \mathrm{~A}$ & 0.9700 & $\mathrm{C} 33 \mathrm{~B}-\mathrm{C} 34 \mathrm{~B}$ & $1.378(5)$ \\
\hline $\mathrm{C} 5 \mathrm{~B}-\mathrm{H} 5 \mathrm{~B}$ & 0.9700 & $\mathrm{C} 33 \mathrm{~B}-\mathrm{H} 33 \mathrm{~B}$ & 0.9300 \\
\hline $\mathrm{C} 5 \mathrm{~A}-\mathrm{S} 1 \mathrm{~A}$ & $1.822(3)$ & $\mathrm{C} 33 \mathrm{~A}-\mathrm{C} 34 \mathrm{~A}$ & $1.368(5)$ \\
\hline $\mathrm{C} 5 \mathrm{~A}-\mathrm{H} 5 \mathrm{~A} 1$ & 0.9700 & $\mathrm{C} 33 \mathrm{~A}-\mathrm{H} 33 \mathrm{~A}$ & 0.9300 \\
\hline $\mathrm{C} 5 \mathrm{~A}-\mathrm{H} 5 \mathrm{~A} 2$ & 0.9700 & $\mathrm{C} 34 \mathrm{~B}-\mathrm{C} 35 \mathrm{~B}$ & $1.377(5)$ \\
\hline $\mathrm{C} 6 \mathrm{~B}-\mathrm{N} 1 \mathrm{~B}$ & $1.435(4)$ & $\mathrm{C} 34 \mathrm{~B}-\mathrm{H} 34 \mathrm{~B}$ & 0.9300 \\
\hline $\mathrm{C} 6 \mathrm{~B}-\mathrm{S} 1 \mathrm{~B}$ & $1.814(3)$ & $\mathrm{C} 34 \mathrm{~A}-\mathrm{C} 35 \mathrm{~A}$ & $1.371(5)$ \\
\hline $\mathrm{C} 6 \mathrm{~B}-\mathrm{H} 6 \mathrm{~A}$ & 0.9700 & $\mathrm{C} 34 \mathrm{~A}-\mathrm{H} 34 \mathrm{~A}$ & 0.9300 \\
\hline $\mathrm{C} 6 \mathrm{~B}-\mathrm{H} 6 \mathrm{~B}$ & 0.9700 & $\mathrm{C} 35 \mathrm{~B}-\mathrm{C} 36 \mathrm{~B}$ & $1.383(4)$ \\
\hline $\mathrm{C} 6 \mathrm{~A}-\mathrm{N} 1 \mathrm{~A}$ & $1.437(4)$ & $\mathrm{C} 35 \mathrm{~B}-\mathrm{H} 35 \mathrm{~B}$ & 0.9300 \\
\hline
\end{tabular}




\begin{tabular}{|c|c|}
\hline $\mathrm{C} 6 \mathrm{~A}-\mathrm{S} 1 \mathrm{~A}$ & $1.815(3)$ \\
\hline $\mathrm{C} 6 \mathrm{~A}-\mathrm{H} 6 \mathrm{~A} 1$ & 0.9700 \\
\hline $\mathrm{C} 6 \mathrm{~A}-\mathrm{H} 6 \mathrm{~A} 2$ & 0.9700 \\
\hline $\mathrm{C} 11 \mathrm{~B}-\mathrm{C} 16 \mathrm{~B}$ & $1.383(4)$ \\
\hline $\mathrm{C} 11 \mathrm{~B}-\mathrm{C} 12 \mathrm{~B}$ & $1.384(4)$ \\
\hline $\mathrm{C} 11 \mathrm{~A}-\mathrm{C} 12 \mathrm{~A}$ & $1.382(4)$ \\
\hline $\mathrm{C} 11 \mathrm{~A}-\mathrm{C} 16 \mathrm{~A}$ & $1.387(4)$ \\
\hline $\mathrm{C} 12 \mathrm{~B}-\mathrm{C} 13 \mathrm{~B}$ & $1.387(4)$ \\
\hline $\mathrm{C} 12 \mathrm{~B}-\mathrm{H} 12 \mathrm{~B}$ & 0.9300 \\
\hline $\mathrm{C} 12 \mathrm{~A}-\mathrm{C} 13 \mathrm{~A}$ & $1.385(4)$ \\
\hline $\mathrm{C} 12 \mathrm{~A}-\mathrm{H} 12 \mathrm{~A}$ & 0.9300 \\
\hline $\mathrm{C} 13 \mathrm{~B}-\mathrm{C} 14 \mathrm{~B}$ & $1.357(5)$ \\
\hline $\mathrm{C} 13 \mathrm{~B}-\mathrm{H} 13 \mathrm{~B}$ & 0.9300 \\
\hline $\mathrm{C} 13 \mathrm{~A}-\mathrm{C} 14 \mathrm{~A}$ & $1.358(5)$ \\
\hline $\mathrm{C} 13 \mathrm{~A}-\mathrm{H} 13 \mathrm{~A}$ & 0.9300 \\
\hline $\mathrm{C} 14 \mathrm{~B}-\mathrm{C} 15 \mathrm{~B}$ & $1.363(5)$ \\
\hline $\mathrm{C} 14 \mathrm{~B}-\mathrm{C} 11 \mathrm{~B}$ & $1.735(3)$ \\
\hline $\mathrm{C} 14 \mathrm{~A}-\mathrm{C} 15 \mathrm{~A}$ & $1.363(5)$ \\
\hline $\mathrm{C} 14 \mathrm{~A}-\mathrm{Cl1} \mathrm{A}$ & $1.740(3)$ \\
\hline $\mathrm{C} 15 \mathrm{~B}-\mathrm{C} 16 \mathrm{~B}$ & $1.389(5)$ \\
\hline $\mathrm{C} 15 \mathrm{~B}-\mathrm{H} 15 \mathrm{~B}$ & 0.9300 \\
\hline $\mathrm{C} 15 \mathrm{~A}-\mathrm{C} 16 \mathrm{~A}$ & $1.381(4)$ \\
\hline $\mathrm{C} 15 \mathrm{~A}-\mathrm{H} 15 \mathrm{~A}$ & 0.9300 \\
\hline $\mathrm{C} 16 \mathrm{~B}-\mathrm{H} 16 \mathrm{~B}$ & 0.9300 \\
\hline $\mathrm{C} 16 \mathrm{~A}-\mathrm{H} 16 \mathrm{~A}$ & 0.9300 \\
\hline $\mathrm{C} 21 \mathrm{~B}-\mathrm{O} 1 \mathrm{~B}$ & $1.207(3)$ \\
\hline $\mathrm{C} 21 \mathrm{~B}-\mathrm{C} 22 \mathrm{~B}$ & $1.476(4)$ \\
\hline $\mathrm{C} 21 \mathrm{~A}-\mathrm{O} 1 \mathrm{~A}$ & $1.203(3)$ \\
\hline $\mathrm{C} 21 \mathrm{~A}-\mathrm{C} 22 \mathrm{~A}$ & $1.492(4)$ \\
\hline $\mathrm{C} 22 \mathrm{~B}-\mathrm{C} 23 \mathrm{~B}$ & $1.389(4)$ \\
\hline $\mathrm{C} 22 \mathrm{~B}-\mathrm{C} 26 \mathrm{~B}$ & $1.392(4)$ \\
\hline $\mathrm{C} 22 \mathrm{~A}-\mathrm{C} 23 \mathrm{~A}$ & $1.384(4)$ \\
\hline $\mathrm{C} 22 \mathrm{~A}-\mathrm{C} 26 \mathrm{~A}$ & $1.387(4)$ \\
\hline $\mathrm{C} 23 \mathrm{~B}-\mathrm{C} 24 \mathrm{~B}$ & $1.363(4)$ \\
\hline $\mathrm{C} 23 \mathrm{~B}-\mathrm{H} 23 \mathrm{~B}$ & 0.9300 \\
\hline $\mathrm{C} 23 \mathrm{~A}-\mathrm{C} 24 \mathrm{~A}$ & $1.363(4)$ \\
\hline $\mathrm{C} 23 \mathrm{~A}-\mathrm{H} 23 \mathrm{~A}$ & 0.9300 \\
\hline $\mathrm{C} 24 \mathrm{~B}-\mathrm{C} 25 \mathrm{~B}$ & $1.384(4)$ \\
\hline $\mathrm{C} 24 \mathrm{~B}-\mathrm{H} 24 \mathrm{~B}$ & 0.9300 \\
\hline $\mathrm{C} 24 \mathrm{~A}-\mathrm{C} 25 \mathrm{~A}$ & $1.382(5)$ \\
\hline $\mathrm{C} 11 \mathrm{~B}-\mathrm{C} 1 \mathrm{~B}-\mathrm{C} 4 \mathrm{~B}$ & $116.3(2)$ \\
\hline $\mathrm{C} 11 \mathrm{~B}-\mathrm{C} 1 \mathrm{~B}-\mathrm{C} 2 \mathrm{~B}$ & $115.1(2)$ \\
\hline $\mathrm{C} 4 \mathrm{~B}-\mathrm{C} 1 \mathrm{~B}-\mathrm{C} 2 \mathrm{~B}$ & $105.5(2)$ \\
\hline $\mathrm{C} 11 \mathrm{~B}-\mathrm{C} 1 \mathrm{~B}-\mathrm{H} 1 \mathrm{~B}$ & 106.4 \\
\hline $\mathrm{C} 4 \mathrm{~B}-\mathrm{C} 1 \mathrm{~B}-\mathrm{H} 1 \mathrm{~B}$ & 106.4 \\
\hline $\mathrm{C} 2 \mathrm{~B}-\mathrm{C} 1 \mathrm{~B}-\mathrm{H} 1 \mathrm{~B}$ & 106.4 \\
\hline $\mathrm{C} 11 \mathrm{~A}-\mathrm{C} 1 \mathrm{~A}-\mathrm{C} 4 \mathrm{~A}$ & $114.8(2)$ \\
\hline
\end{tabular}

\begin{tabular}{|c|c|}
\hline $\mathrm{C} 35 \mathrm{~A}-\mathrm{C} 36 \mathrm{~A}$ & $1.376(4)$ \\
\hline $\mathrm{C} 35 \mathrm{~A}-\mathrm{H} 35 \mathrm{~A}$ & 0.9300 \\
\hline $\mathrm{C} 36 \mathrm{~B}-\mathrm{N} 3 \mathrm{~B}$ & $1.401(4)$ \\
\hline $\mathrm{C} 36 \mathrm{~A}-\mathrm{N} 3 \mathrm{~A}$ & $1.389(4)$ \\
\hline $\mathrm{C} 37 \mathrm{~B}-\mathrm{O} 2 \mathrm{~B}$ & $1.218(3)$ \\
\hline $\mathrm{C} 37 \mathrm{~B}-\mathrm{N} 3 \mathrm{~B}$ & $1.360(4)$ \\
\hline $\mathrm{C} 37 \mathrm{~A}-\mathrm{O} 2 \mathrm{~A}$ & $1.226(3)$ \\
\hline $\mathrm{C} 37 \mathrm{~A}-\mathrm{N} 3 \mathrm{~A}$ & $1.348(4)$ \\
\hline $\mathrm{C} 51 \mathrm{~B}-\mathrm{C} 56 \mathrm{~B}$ & $1.377(4)$ \\
\hline $\mathrm{C} 51 \mathrm{~B}-\mathrm{C} 52 \mathrm{~B}$ & $1.387(4)$ \\
\hline $\mathrm{C} 51 \mathrm{~A}-\mathrm{C} 56 \mathrm{~A}$ & $1.374(5)$ \\
\hline $\mathrm{C} 51 \mathrm{~A}-\mathrm{C} 52 \mathrm{~A}$ & $1.380(5)$ \\
\hline $\mathrm{C} 52 \mathrm{~B}-\mathrm{C} 53 \mathrm{~B}$ & $1.370(5)$ \\
\hline $\mathrm{C} 52 \mathrm{~B}-\mathrm{H} 52 \mathrm{~B}$ & 0.9300 \\
\hline $\mathrm{C} 52 \mathrm{~A}-\mathrm{C} 53 \mathrm{~A}$ & $1.381(5)$ \\
\hline $\mathrm{C} 52 \mathrm{~A}-\mathrm{H} 52 \mathrm{~A}$ & 0.9300 \\
\hline $\mathrm{C} 53 \mathrm{~B}-\mathrm{C} 54 \mathrm{~B}$ & $1.370(5)$ \\
\hline $\mathrm{C} 53 \mathrm{~B}-\mathrm{H} 53 \mathrm{~B}$ & 0.9300 \\
\hline $\mathrm{C} 53 \mathrm{~A}-\mathrm{C} 54 \mathrm{~A}$ & $1.360(6)$ \\
\hline $\mathrm{C} 53 \mathrm{~A}-\mathrm{H} 53 \mathrm{~A}$ & 0.9300 \\
\hline $\mathrm{C} 54 \mathrm{~B}-\mathrm{O} 3 \mathrm{~B}$ & $1.364(4)$ \\
\hline $\mathrm{C} 54 \mathrm{~B}-\mathrm{C} 55 \mathrm{~B}$ & $1.376(4)$ \\
\hline $\mathrm{C} 54 \mathrm{~A}-\mathrm{O} 3 \mathrm{~A}$ & $1.357(4)$ \\
\hline $\mathrm{C} 54 \mathrm{~A}-\mathrm{C} 55 \mathrm{~A}$ & $1.377(5)$ \\
\hline $\mathrm{C} 55 \mathrm{~B}-\mathrm{C} 56 \mathrm{~B}$ & $1.380(4)$ \\
\hline $\mathrm{C} 55 \mathrm{~B}-\mathrm{H} 55 \mathrm{~B}$ & 0.9300 \\
\hline $\mathrm{C} 55 \mathrm{~A}-\mathrm{C} 56 \mathrm{~A}$ & $1.373(5)$ \\
\hline $\mathrm{C} 55 \mathrm{~A}-\mathrm{H} 55 \mathrm{~A}$ & 0.9300 \\
\hline $\mathrm{C} 56 \mathrm{~B}-\mathrm{H} 56 \mathrm{~B}$ & 0.9300 \\
\hline $\mathrm{C} 56 \mathrm{~A}-\mathrm{H} 56 \mathrm{~A}$ & 0.9300 \\
\hline $\mathrm{N} 3 \mathrm{~B}-\mathrm{H} 3 \mathrm{~B}$ & $0.90(4)$ \\
\hline $\mathrm{N} 3 \mathrm{~A}-\mathrm{H} 3 \mathrm{~A}$ & $0.84(4)$ \\
\hline $\mathrm{O} 3 \mathrm{~B}-\mathrm{C} 57 \mathrm{~B}$ & $1.405(4)$ \\
\hline $\mathrm{O} 3 \mathrm{~A}-\mathrm{C} 57 \mathrm{~A}$ & $1.411(5)$ \\
\hline C57B-H57A & 0.9600 \\
\hline $\mathrm{C} 57 \mathrm{~B}-\mathrm{H} 57 \mathrm{C}$ & 0.9600 \\
\hline $\mathrm{C} 57 \mathrm{~B}-\mathrm{H} 57 \mathrm{~B}$ & 0.9600 \\
\hline C57A-H57D & 0.9600 \\
\hline C57A-H57E & 0.9600 \\
\hline $\mathrm{C} 57 \mathrm{~A}-\mathrm{H} 57 \mathrm{~F}$ & 0.9600 \\
\hline $\mathrm{N} 2 \mathrm{~B}-\mathrm{C} 26 \mathrm{~B}-\mathrm{C} 22 \mathrm{~B}$ & $122.9(3)$ \\
\hline $\mathrm{N} 2 \mathrm{~B}-\mathrm{C} 26 \mathrm{~B}-\mathrm{C} 27 \mathrm{~B}$ & $116.7(3)$ \\
\hline $\mathrm{C} 22 \mathrm{~B}-\mathrm{C} 26 \mathrm{~B}-\mathrm{C} 27 \mathrm{~B}$ & $120.4(3)$ \\
\hline $\mathrm{N} 2 \mathrm{~A}-\mathrm{C} 26 \mathrm{~A}-\mathrm{C} 22 \mathrm{~A}$ & $122.7(3)$ \\
\hline $\mathrm{N} 2 \mathrm{~A}-\mathrm{C} 26 \mathrm{~A}-\mathrm{C} 27 \mathrm{~A}$ & $117.1(3)$ \\
\hline $\mathrm{C} 22 \mathrm{~A}-\mathrm{C} 26 \mathrm{~A}-\mathrm{C} 27 \mathrm{~A}$ & $120.2(3)$ \\
\hline $\mathrm{C} 26 \mathrm{~B}-\mathrm{C} 27 \mathrm{~B}-\mathrm{C} 28 \mathrm{~B}$ & $112.6(2)$ \\
\hline
\end{tabular}




\begin{tabular}{|c|c|}
\hline $\mathrm{C} 11 \mathrm{~A}-\mathrm{C} 1 \mathrm{~A}-\mathrm{C} 2 \mathrm{~A}$ & $116.2(2)$ \\
\hline $\mathrm{C} 4 \mathrm{~A}-\mathrm{C} 1 \mathrm{~A}-\mathrm{C} 2 \mathrm{~A}$ & $104.9(2)$ \\
\hline $\mathrm{C} 11 \mathrm{~A}-\mathrm{C} 1 \mathrm{~A}-\mathrm{H} 1 \mathrm{~A}$ & 106.8 \\
\hline $\mathrm{C} 4 \mathrm{~A}-\mathrm{C} 1 \mathrm{~A}-\mathrm{H} 1 \mathrm{~A}$ & 106.8 \\
\hline $\mathrm{C} 2 \mathrm{~A}-\mathrm{C} 1 \mathrm{~A}-\mathrm{H} 1 \mathrm{~A}$ & 106.8 \\
\hline $\mathrm{C} 28 \mathrm{~B}-\mathrm{C} 2 \mathrm{~B}-\mathrm{C} 21 \mathrm{~B}$ & $106.4(2)$ \\
\hline $\mathrm{C} 28 \mathrm{~B}-\mathrm{C} 2 \mathrm{~B}-\mathrm{C} 1 \mathrm{~B}$ & $115.1(2)$ \\
\hline $\mathrm{C} 21 \mathrm{~B}-\mathrm{C} 2 \mathrm{~B}-\mathrm{C} 1 \mathrm{~B}$ & $109.1(2)$ \\
\hline $\mathrm{C} 28 \mathrm{~B}-\mathrm{C} 2 \mathrm{~B}-\mathrm{C} 3 \mathrm{~B}$ & $113.6(2)$ \\
\hline $\mathrm{C} 21 \mathrm{~B}-\mathrm{C} 2 \mathrm{~B}-\mathrm{C} 3 \mathrm{~B}$ & $109.0(2)$ \\
\hline $\mathrm{C} 1 \mathrm{~B}-\mathrm{C} 2 \mathrm{~B}-\mathrm{C} 3 \mathrm{~B}$ & $103.5(2)$ \\
\hline $\mathrm{C} 28 \mathrm{~A}-\mathrm{C} 2 \mathrm{~A}-\mathrm{C} 21 \mathrm{~A}$ & $107.1(2)$ \\
\hline $\mathrm{C} 28 \mathrm{~A}-\mathrm{C} 2 \mathrm{~A}-\mathrm{C} 1 \mathrm{~A}$ & $114.8(2)$ \\
\hline $\mathrm{C} 21 \mathrm{~A}-\mathrm{C} 2 \mathrm{~A}-\mathrm{C} 1 \mathrm{~A}$ & $109.8(2)$ \\
\hline $\mathrm{C} 28 \mathrm{~A}-\mathrm{C} 2 \mathrm{~A}-\mathrm{C} 3 \mathrm{~A}$ & $114.6(2)$ \\
\hline $\mathrm{C} 21 \mathrm{~A}-\mathrm{C} 2 \mathrm{~A}-\mathrm{C} 3 \mathrm{~A}$ & $107.3(2)$ \\
\hline $\mathrm{C} 1 \mathrm{~A}-\mathrm{C} 2 \mathrm{~A}-\mathrm{C} 3 \mathrm{~A}$ & $103.0(2)$ \\
\hline $\mathrm{N} 1 \mathrm{~B}-\mathrm{C} 3 \mathrm{~B}-\mathrm{C} 31 \mathrm{~B}$ & $110.7(2)$ \\
\hline $\mathrm{N} 1 \mathrm{~B}-\mathrm{C} 3 \mathrm{~B}-\mathrm{C} 37 \mathrm{~B}$ & $112.4(2)$ \\
\hline $\mathrm{C} 31 \mathrm{~B}-\mathrm{C} 3 \mathrm{~B}-\mathrm{C} 37 \mathrm{~B}$ & $101.7(2)$ \\
\hline $\mathrm{N} 1 \mathrm{~B}-\mathrm{C} 3 \mathrm{~B}-\mathrm{C} 2 \mathrm{~B}$ & $100.6(2)$ \\
\hline $\mathrm{C} 31 \mathrm{~B}-\mathrm{C} 3 \mathrm{~B}-\mathrm{C} 2 \mathrm{~B}$ & $121.1(2)$ \\
\hline $\mathrm{C} 37 \mathrm{~B}-\mathrm{C} 3 \mathrm{~B}-\mathrm{C} 2 \mathrm{~B}$ & $110.8(2)$ \\
\hline $\mathrm{N} 1 \mathrm{~A}-\mathrm{C} 3 \mathrm{~A}-\mathrm{C} 31 \mathrm{~A}$ & $112.7(2)$ \\
\hline $\mathrm{N} 1 \mathrm{~A}-\mathrm{C} 3 \mathrm{~A}-\mathrm{C} 37 \mathrm{~A}$ & $113.2(2)$ \\
\hline $\mathrm{C} 31 \mathrm{~A}-\mathrm{C} 3 \mathrm{~A}-\mathrm{C} 37 \mathrm{~A}$ & $101.4(2)$ \\
\hline $\mathrm{N} 1 \mathrm{~A}-\mathrm{C} 3 \mathrm{~A}-\mathrm{C} 2 \mathrm{~A}$ & $101.2(2)$ \\
\hline $\mathrm{C} 31 \mathrm{~A}-\mathrm{C} 3 \mathrm{~A}-\mathrm{C} 2 \mathrm{~A}$ & $117.9(2)$ \\
\hline $\mathrm{C} 37 \mathrm{~A}-\mathrm{C} 3 \mathrm{~A}-\mathrm{C} 2 \mathrm{~A}$ & $110.9(2)$ \\
\hline $\mathrm{N} 1 \mathrm{~B}-\mathrm{C} 4 \mathrm{~B}-\mathrm{C} 5 \mathrm{~B}$ & $104.0(2)$ \\
\hline $\mathrm{N} 1 \mathrm{~B}-\mathrm{C} 4 \mathrm{~B}-\mathrm{C} 1 \mathrm{~B}$ & $101.4(2)$ \\
\hline $\mathrm{C} 5 \mathrm{~B}-\mathrm{C} 4 \mathrm{~B}-\mathrm{C} 1 \mathrm{~B}$ & $119.6(3)$ \\
\hline $\mathrm{N} 1 \mathrm{~B}-\mathrm{C} 4 \mathrm{~B}-\mathrm{H} 4 \mathrm{~B}$ & 110.3 \\
\hline $\mathrm{C} 5 \mathrm{~B}-\mathrm{C} 4 \mathrm{~B}-\mathrm{H} 4 \mathrm{~B}$ & 110.3 \\
\hline $\mathrm{C} 1 \mathrm{~B}-\mathrm{C} 4 \mathrm{~B}-\mathrm{H} 4 \mathrm{~B}$ & 110.3 \\
\hline $\mathrm{N} 1 \mathrm{~A}-\mathrm{C} 4 \mathrm{~A}-\mathrm{C} 5 \mathrm{~A}$ & $105.0(2)$ \\
\hline $\mathrm{N} 1 \mathrm{~A}-\mathrm{C} 4 \mathrm{~A}-\mathrm{C} 1 \mathrm{~A}$ & $101.1(2)$ \\
\hline $\mathrm{C} 5 \mathrm{~A}-\mathrm{C} 4 \mathrm{~A}-\mathrm{C} 1 \mathrm{~A}$ & $119.9(3)$ \\
\hline $\mathrm{N} 1 \mathrm{~A}-\mathrm{C} 4 \mathrm{~A}-\mathrm{H} 4 \mathrm{~A}$ & 110.0 \\
\hline $\mathrm{C} 5 \mathrm{~A}-\mathrm{C} 4 \mathrm{~A}-\mathrm{H} 4 \mathrm{~A}$ & 110.0 \\
\hline $\mathrm{C} 1 \mathrm{~A}-\mathrm{C} 4 \mathrm{~A}-\mathrm{H} 4 \mathrm{~A}$ & 110.0 \\
\hline $\mathrm{C} 4 \mathrm{~B}-\mathrm{C} 5 \mathrm{~B}-\mathrm{S} 1 \mathrm{~B}$ & $104.5(2)$ \\
\hline $\mathrm{C} 4 \mathrm{~B}-\mathrm{C} 5 \mathrm{~B}-\mathrm{H} 5 \mathrm{~A}$ & 110.9 \\
\hline $\mathrm{S} 1 \mathrm{~B}-\mathrm{C} 5 \mathrm{~B}-\mathrm{H} 5 \mathrm{~A}$ & 110.9 \\
\hline $\mathrm{C} 4 \mathrm{~B}-\mathrm{C} 5 \mathrm{~B}-\mathrm{H} 5 \mathrm{~B}$ & 110.9 \\
\hline $\mathrm{S} 1 \mathrm{~B}-\mathrm{C} 5 \mathrm{~B}-\mathrm{H} 5 \mathrm{~B}$ & 110.9 \\
\hline $\mathrm{H} 5 \mathrm{~A}-\mathrm{C} 5 \mathrm{~B}-\mathrm{H} 5 \mathrm{~B}$ & 108.9 \\
\hline $\mathrm{C} 4 \mathrm{~A}-\mathrm{C} 5 \mathrm{~A}-\mathrm{S} 1 \mathrm{~A}$ & $104.8(2)$ \\
\hline
\end{tabular}

\begin{tabular}{|c|c|}
\hline $\mathrm{C} 26 \mathrm{~B}-\mathrm{C} 27 \mathrm{~B}-\mathrm{H} 27 \mathrm{~A}$ & 109.1 \\
\hline $\mathrm{C} 28 \mathrm{~B}-\mathrm{C} 27 \mathrm{~B}-\mathrm{H} 27 \mathrm{~A}$ & 109.1 \\
\hline $\mathrm{C} 26 \mathrm{~B}-\mathrm{C} 27 \mathrm{~B}-\mathrm{H} 27 \mathrm{~B}$ & 109.1 \\
\hline $\mathrm{C} 28 \mathrm{~B}-\mathrm{C} 27 \mathrm{~B}-\mathrm{H} 27 \mathrm{~B}$ & 109.1 \\
\hline $\mathrm{H} 27 \mathrm{~A}-\mathrm{C} 27 \mathrm{~B}-\mathrm{H} 27 \mathrm{~B}$ & 107.8 \\
\hline $\mathrm{C} 26 \mathrm{~A}-\mathrm{C} 27 \mathrm{~A}-\mathrm{C} 28 \mathrm{~A}$ & $113.2(2)$ \\
\hline $\mathrm{C} 26 \mathrm{~A}-\mathrm{C} 27 \mathrm{~A}-\mathrm{H} 27 \mathrm{C}$ & 108.9 \\
\hline $\mathrm{C} 28 \mathrm{~A}-\mathrm{C} 27 \mathrm{~A}-\mathrm{H} 27 \mathrm{C}$ & 108.9 \\
\hline $\mathrm{C} 26 \mathrm{~A}-\mathrm{C} 27 \mathrm{~A}-\mathrm{H} 27 \mathrm{D}$ & 108.9 \\
\hline $\mathrm{C} 28 \mathrm{~A}-\mathrm{C} 27 \mathrm{~A}-\mathrm{H} 27 \mathrm{D}$ & 108.9 \\
\hline $\mathrm{H} 27 \mathrm{C}-\mathrm{C} 27 \mathrm{~A}-\mathrm{H} 27 \mathrm{D}$ & 107.7 \\
\hline $\mathrm{C} 27 \mathrm{~B}-\mathrm{C} 28 \mathrm{~B}-\mathrm{C} 2 \mathrm{~B}$ & $113.2(2)$ \\
\hline $\mathrm{C} 27 \mathrm{~B}-\mathrm{C} 28 \mathrm{~B}-\mathrm{H} 28 \mathrm{~A}$ & 108.9 \\
\hline $\mathrm{C} 2 \mathrm{~B}-\mathrm{C} 28 \mathrm{~B}-\mathrm{H} 28 \mathrm{~A}$ & 108.9 \\
\hline $\mathrm{C} 27 \mathrm{~B}-\mathrm{C} 28 \mathrm{~B}-\mathrm{H} 28 \mathrm{~B}$ & 108.9 \\
\hline $\mathrm{C} 2 \mathrm{~B}-\mathrm{C} 28 \mathrm{~B}-\mathrm{H} 28 \mathrm{~B}$ & 108.9 \\
\hline $\mathrm{H} 28 \mathrm{~A}-\mathrm{C} 28 \mathrm{~B}-\mathrm{H} 28 \mathrm{~B}$ & 107.7 \\
\hline $\mathrm{C} 2 \mathrm{~A}-\mathrm{C} 28 \mathrm{~A}-\mathrm{C} 27 \mathrm{~A}$ & $115.4(2)$ \\
\hline $\mathrm{C} 2 \mathrm{~A}-\mathrm{C} 28 \mathrm{~A}-\mathrm{H} 28 \mathrm{C}$ & 108.4 \\
\hline $\mathrm{C} 27 \mathrm{~A}-\mathrm{C} 28 \mathrm{~A}-\mathrm{H} 28 \mathrm{C}$ & 108.4 \\
\hline $\mathrm{C} 2 \mathrm{~A}-\mathrm{C} 28 \mathrm{~A}-\mathrm{H} 28 \mathrm{D}$ & 108.4 \\
\hline $\mathrm{C} 27 \mathrm{~A}-\mathrm{C} 28 \mathrm{~A}-\mathrm{H} 28 \mathrm{D}$ & 108.4 \\
\hline $\mathrm{H} 28 \mathrm{C}-\mathrm{C} 28 \mathrm{~A}-\mathrm{H} 28 \mathrm{D}$ & 107.5 \\
\hline $\mathrm{C} 36 \mathrm{~B}-\mathrm{C} 31 \mathrm{~B}-\mathrm{C} 32 \mathrm{~B}$ & $119.6(3)$ \\
\hline $\mathrm{C} 36 \mathrm{~B}-\mathrm{C} 31 \mathrm{~B}-\mathrm{C} 3 \mathrm{~B}$ & $108.8(3)$ \\
\hline $\mathrm{C} 32 \mathrm{~B}-\mathrm{C} 31 \mathrm{~B}-\mathrm{C} 3 \mathrm{~B}$ & $131.1(3)$ \\
\hline $\mathrm{C} 32 \mathrm{~A}-\mathrm{C} 31 \mathrm{~A}-\mathrm{C} 36 \mathrm{~A}$ & $120.2(3)$ \\
\hline $\mathrm{C} 32 \mathrm{~A}-\mathrm{C} 31 \mathrm{~A}-\mathrm{C} 3 \mathrm{~A}$ & $131.1(3)$ \\
\hline $\mathrm{C} 36 \mathrm{~A}-\mathrm{C} 31 \mathrm{~A}-\mathrm{C} 3 \mathrm{~A}$ & $108.6(3)$ \\
\hline $\mathrm{C} 31 \mathrm{~B}-\mathrm{C} 32 \mathrm{~B}-\mathrm{C} 33 \mathrm{~B}$ & $118.8(3)$ \\
\hline $\mathrm{C} 31 \mathrm{~B}-\mathrm{C} 32 \mathrm{~B}-\mathrm{H} 32 \mathrm{~B}$ & 120.6 \\
\hline $\mathrm{C} 33 \mathrm{~B}-\mathrm{C} 32 \mathrm{~B}-\mathrm{H} 32 \mathrm{~B}$ & 120.6 \\
\hline $\mathrm{C} 31 \mathrm{~A}-\mathrm{C} 32 \mathrm{~A}-\mathrm{C} 33 \mathrm{~A}$ & $118.6(3)$ \\
\hline $\mathrm{C} 31 \mathrm{~A}-\mathrm{C} 32 \mathrm{~A}-\mathrm{H} 32 \mathrm{~A}$ & 120.7 \\
\hline $\mathrm{C} 33 \mathrm{~A}-\mathrm{C} 32 \mathrm{~A}-\mathrm{H} 32 \mathrm{~A}$ & 120.7 \\
\hline $\mathrm{C} 34 \mathrm{~B}-\mathrm{C} 33 \mathrm{~B}-\mathrm{C} 32 \mathrm{~B}$ & $120.3(3)$ \\
\hline $\mathrm{C} 34 \mathrm{~B}-\mathrm{C} 33 \mathrm{~B}-\mathrm{H} 33 \mathrm{~B}$ & 119.9 \\
\hline $\mathrm{C} 32 \mathrm{~B}-\mathrm{C} 33 \mathrm{~B}-\mathrm{H} 33 \mathrm{~B}$ & 119.9 \\
\hline $\mathrm{C} 34 \mathrm{~A}-\mathrm{C} 33 \mathrm{~A}-\mathrm{C} 32 \mathrm{~A}$ & $120.3(4)$ \\
\hline $\mathrm{C} 34 \mathrm{~A}-\mathrm{C} 33 \mathrm{~A}-\mathrm{H} 33 \mathrm{~A}$ & 119.9 \\
\hline $\mathrm{C} 32 \mathrm{~A}-\mathrm{C} 33 \mathrm{~A}-\mathrm{H} 33 \mathrm{~A}$ & 119.9 \\
\hline $\mathrm{C} 35 \mathrm{~B}-\mathrm{C} 34 \mathrm{~B}-\mathrm{C} 33 \mathrm{~B}$ & $121.8(3)$ \\
\hline $\mathrm{C} 35 \mathrm{~B}-\mathrm{C} 34 \mathrm{~B}-\mathrm{H} 34 \mathrm{~B}$ & 119.1 \\
\hline $\mathrm{C} 33 \mathrm{~B}-\mathrm{C} 34 \mathrm{~B}-\mathrm{H} 34 \mathrm{~B}$ & 119.1 \\
\hline $\mathrm{C} 33 \mathrm{~A}-\mathrm{C} 34 \mathrm{~A}-\mathrm{C} 35 \mathrm{~A}$ & $121.5(3)$ \\
\hline $\mathrm{C} 33 \mathrm{~A}-\mathrm{C} 34 \mathrm{~A}-\mathrm{H} 34 \mathrm{~A}$ & 119.2 \\
\hline $\mathrm{C} 35 \mathrm{~A}-\mathrm{C} 34 \mathrm{~A}-\mathrm{H} 34 \mathrm{~A}$ & 119.2 \\
\hline $\mathrm{C} 34 \mathrm{~B}-\mathrm{C} 35 \mathrm{~B}-\mathrm{C} 36 \mathrm{~B}$ & $116.9(3)$ \\
\hline
\end{tabular}




\begin{tabular}{|c|c|}
\hline $\mathrm{C} 4 \mathrm{~A}-\mathrm{C} 5 \mathrm{~A}-\mathrm{H} 5 \mathrm{~A} 1$ & 110.8 \\
\hline $\mathrm{S} 1 \mathrm{~A}-\mathrm{C} 5 \mathrm{~A}-\mathrm{H} 5 \mathrm{~A} 1$ & 110.8 \\
\hline $\mathrm{C} 4 \mathrm{~A}-\mathrm{C} 5 \mathrm{~A}-\mathrm{H} 5 \mathrm{~A} 2$ & 110.8 \\
\hline $\mathrm{S} 1 \mathrm{~A}-\mathrm{C} 5 \mathrm{~A}-\mathrm{H} 5 \mathrm{~A} 2$ & 110.8 \\
\hline $\mathrm{H} 5 \mathrm{~A} 1-\mathrm{C} 5 \mathrm{~A}-\mathrm{H} 5 \mathrm{~A} 2$ & 108.9 \\
\hline $\mathrm{N} 1 \mathrm{~B}-\mathrm{C} 6 \mathrm{~B}-\mathrm{S} 1 \mathrm{~B}$ & $103.7(2)$ \\
\hline $\mathrm{N} 1 \mathrm{~B}-\mathrm{C} 6 \mathrm{~B}-\mathrm{H} 6 \mathrm{~A}$ & 111.0 \\
\hline $\mathrm{S} 1 \mathrm{~B}-\mathrm{C} 6 \mathrm{~B}-\mathrm{H} 6 \mathrm{~A}$ & 111.0 \\
\hline $\mathrm{N} 1 \mathrm{~B}-\mathrm{C} 6 \mathrm{~B}-\mathrm{H} 6 \mathrm{~B}$ & 111.0 \\
\hline $\mathrm{S} 1 \mathrm{~B}-\mathrm{C} 6 \mathrm{~B}-\mathrm{H} 6 \mathrm{~B}$ & 111.0 \\
\hline $\mathrm{H} 6 \mathrm{~A}-\mathrm{C} 6 \mathrm{~B}-\mathrm{H} 6 \mathrm{~B}$ & 109.0 \\
\hline $\mathrm{N} 1 \mathrm{~A}-\mathrm{C} 6 \mathrm{~A}-\mathrm{S} 1 \mathrm{~A}$ & $103.5(2)$ \\
\hline $\mathrm{N} 1 \mathrm{~A}-\mathrm{C} 6 \mathrm{~A}-\mathrm{H} 6 \mathrm{~A} 1$ & 111.1 \\
\hline $\mathrm{S} 1 \mathrm{~A}-\mathrm{C} 6 \mathrm{~A}-\mathrm{H} 6 \mathrm{~A} 1$ & 111.1 \\
\hline $\mathrm{N} 1 \mathrm{~A}-\mathrm{C} 6 \mathrm{~A}-\mathrm{H} 6 \mathrm{~A} 2$ & 111.1 \\
\hline $\mathrm{S} 1 \mathrm{~A}-\mathrm{C} 6 \mathrm{~A}-\mathrm{H} 6 \mathrm{~A} 2$ & 111.1 \\
\hline $\mathrm{H} 6 \mathrm{~A} 1-\mathrm{C} 6 \mathrm{~A}-\mathrm{H} 6 \mathrm{~A} 2$ & 109.0 \\
\hline $\mathrm{C} 16 \mathrm{~B}-\mathrm{C} 11 \mathrm{~B}-\mathrm{C} 12 \mathrm{~B}$ & $117.4(3)$ \\
\hline $\mathrm{C} 16 \mathrm{~B}-\mathrm{C} 11 \mathrm{~B}-\mathrm{C} 1 \mathrm{~B}$ & $119.7(3)$ \\
\hline $\mathrm{C} 12 \mathrm{~B}-\mathrm{C} 11 \mathrm{~B}-\mathrm{C} 1 \mathrm{~B}$ & $122.9(3)$ \\
\hline $\mathrm{C} 12 \mathrm{~A}-\mathrm{C} 11 \mathrm{~A}-\mathrm{C} 16 \mathrm{~A}$ & $117.5(3)$ \\
\hline $\mathrm{C} 12 \mathrm{~A}-\mathrm{C} 11 \mathrm{~A}-\mathrm{C} 1 \mathrm{~A}$ & $122.9(3)$ \\
\hline $\mathrm{C} 16 \mathrm{~A}-\mathrm{C} 11 \mathrm{~A}-\mathrm{C} 1 \mathrm{~A}$ & $119.6(3)$ \\
\hline $\mathrm{C} 11 \mathrm{~B}-\mathrm{C} 12 \mathrm{~B}-\mathrm{C} 13 \mathrm{~B}$ & $121.3(3)$ \\
\hline $\mathrm{C} 11 \mathrm{~B}-\mathrm{C} 12 \mathrm{~B}-\mathrm{H} 12 \mathrm{~B}$ & 119.4 \\
\hline $\mathrm{C} 13 \mathrm{~B}-\mathrm{C} 12 \mathrm{~B}-\mathrm{H} 12 \mathrm{~B}$ & 119.4 \\
\hline $\mathrm{C} 11 \mathrm{~A}-\mathrm{C} 12 \mathrm{~A}-\mathrm{C} 13 \mathrm{~A}$ & $121.3(3)$ \\
\hline $\mathrm{C} 11 \mathrm{~A}-\mathrm{C} 12 \mathrm{~A}-\mathrm{H} 12 \mathrm{~A}$ & 119.4 \\
\hline $\mathrm{C} 13 \mathrm{~A}-\mathrm{C} 12 \mathrm{~A}-\mathrm{H} 12 \mathrm{~A}$ & 119.4 \\
\hline $\mathrm{C} 14 \mathrm{~B}-\mathrm{C} 13 \mathrm{~B}-\mathrm{C} 12 \mathrm{~B}$ & $119.7(3)$ \\
\hline $\mathrm{C} 14 \mathrm{~B}-\mathrm{C} 13 \mathrm{~B}-\mathrm{H} 13 \mathrm{~B}$ & 120.1 \\
\hline $\mathrm{C} 12 \mathrm{~B}-\mathrm{C} 13 \mathrm{~B}-\mathrm{H} 13 \mathrm{~B}$ & 120.1 \\
\hline $\mathrm{C} 14 \mathrm{~A}-\mathrm{C} 13 \mathrm{~A}-\mathrm{C} 12 \mathrm{~A}$ & $119.3(3)$ \\
\hline $\mathrm{C} 14 \mathrm{~A}-\mathrm{C} 13 \mathrm{~A}-\mathrm{H} 13 \mathrm{~A}$ & 120.3 \\
\hline $\mathrm{C} 12 \mathrm{~A}-\mathrm{C} 13 \mathrm{~A}-\mathrm{H} 13 \mathrm{~A}$ & 120.3 \\
\hline $\mathrm{C} 13 \mathrm{~B}-\mathrm{C} 14 \mathrm{~B}-\mathrm{C} 15 \mathrm{~B}$ & $120.8(3)$ \\
\hline $\mathrm{C} 13 \mathrm{~B}-\mathrm{C} 14 \mathrm{~B}-\mathrm{C} 11 \mathrm{~B}$ & $118.9(3)$ \\
\hline $\mathrm{C} 15 \mathrm{~B}-\mathrm{C} 14 \mathrm{~B}-\mathrm{C} 11 \mathrm{~B}$ & $120.3(3)$ \\
\hline $\mathrm{C} 13 \mathrm{~A}-\mathrm{C} 14 \mathrm{~A}-\mathrm{C} 15 \mathrm{~A}$ & $121.3(3)$ \\
\hline $\mathrm{C} 13 \mathrm{~A}-\mathrm{C} 14 \mathrm{~A}-\mathrm{C} 11 \mathrm{~A}$ & $118.8(3)$ \\
\hline $\mathrm{C} 15 \mathrm{~A}-\mathrm{C} 14 \mathrm{~A}-\mathrm{Cl1A}$ & $119.9(3)$ \\
\hline $\mathrm{C} 14 \mathrm{~B}-\mathrm{C} 15 \mathrm{~B}-\mathrm{C} 16 \mathrm{~B}$ & $119.4(3)$ \\
\hline $\mathrm{C} 14 \mathrm{~B}-\mathrm{C} 15 \mathrm{~B}-\mathrm{H} 15 \mathrm{~B}$ & 120.3 \\
\hline $\mathrm{C} 16 \mathrm{~B}-\mathrm{C} 15 \mathrm{~B}-\mathrm{H} 15 \mathrm{~B}$ & 120.3 \\
\hline $\mathrm{C} 14 \mathrm{~A}-\mathrm{C} 15 \mathrm{~A}-\mathrm{C} 16 \mathrm{~A}$ & $119.2(3)$ \\
\hline $\mathrm{C} 14 \mathrm{~A}-\mathrm{C} 15 \mathrm{~A}-\mathrm{H} 15 \mathrm{~A}$ & 120.4 \\
\hline $\mathrm{C} 16 \mathrm{~A}-\mathrm{C} 15 \mathrm{~A}-\mathrm{H} 15 \mathrm{~A}$ & 120.4 \\
\hline $\mathrm{C} 11 \mathrm{~B}-\mathrm{C} 16 \mathrm{~B}-\mathrm{C} 15 \mathrm{~B}$ & $121.4(3)$ \\
\hline
\end{tabular}

\begin{tabular}{|c|c|}
\hline $\mathrm{C} 34 \mathrm{~B}-\mathrm{C} 35 \mathrm{~B}-\mathrm{H} 35 \mathrm{~B}$ & 121.6 \\
\hline $\mathrm{C} 36 \mathrm{~B}-\mathrm{C} 35 \mathrm{~B}-\mathrm{H} 35 \mathrm{~B}$ & 121.6 \\
\hline $\mathrm{C} 34 \mathrm{~A}-\mathrm{C} 35 \mathrm{~A}-\mathrm{C} 36 \mathrm{~A}$ & $118.0(3)$ \\
\hline $\mathrm{C} 34 \mathrm{~A}-\mathrm{C} 35 \mathrm{~A}-\mathrm{H} 35 \mathrm{~A}$ & 121.0 \\
\hline $\mathrm{C} 36 \mathrm{~A}-\mathrm{C} 35 \mathrm{~A}-\mathrm{H} 35 \mathrm{~A}$ & 121.0 \\
\hline $\mathrm{C} 31 \mathrm{~B}-\mathrm{C} 36 \mathrm{~B}-\mathrm{C} 35 \mathrm{~B}$ & $122.6(3)$ \\
\hline $\mathrm{C} 31 \mathrm{~B}-\mathrm{C} 36 \mathrm{~B}-\mathrm{N} 3 \mathrm{~B}$ & $110.6(3)$ \\
\hline $\mathrm{C} 35 \mathrm{~B}-\mathrm{C} 36 \mathrm{~B}-\mathrm{N} 3 \mathrm{~B}$ & $126.7(3)$ \\
\hline $\mathrm{C} 35 \mathrm{~A}-\mathrm{C} 36 \mathrm{~A}-\mathrm{C} 31 \mathrm{~A}$ & $121.3(3)$ \\
\hline $\mathrm{C} 35 \mathrm{~A}-\mathrm{C} 36 \mathrm{~A}-\mathrm{N} 3 \mathrm{~A}$ & $128.4(3)$ \\
\hline $\mathrm{C} 31 \mathrm{~A}-\mathrm{C} 36 \mathrm{~A}-\mathrm{N} 3 \mathrm{~A}$ & $110.3(3)$ \\
\hline $\mathrm{O} 2 \mathrm{~B}-\mathrm{C} 37 \mathrm{~B}-\mathrm{N} 3 \mathrm{~B}$ & $125.4(3)$ \\
\hline $\mathrm{O} 2 \mathrm{~B}-\mathrm{C} 37 \mathrm{~B}-\mathrm{C} 3 \mathrm{~B}$ & $126.4(3)$ \\
\hline $\mathrm{N} 3 \mathrm{~B}-\mathrm{C} 37 \mathrm{~B}-\mathrm{C} 3 \mathrm{~B}$ & $108.2(2)$ \\
\hline $\mathrm{O} 2 \mathrm{~A}-\mathrm{C} 37 \mathrm{~A}-\mathrm{N} 3 \mathrm{~A}$ & $125.3(3)$ \\
\hline $\mathrm{O} 2 \mathrm{~A}-\mathrm{C} 37 \mathrm{~A}-\mathrm{C} 3 \mathrm{~A}$ & $126.8(3)$ \\
\hline $\mathrm{N} 3 \mathrm{~A}-\mathrm{C} 37 \mathrm{~A}-\mathrm{C} 3 \mathrm{~A}$ & $107.9(3)$ \\
\hline $\mathrm{C} 56 \mathrm{~B}-\mathrm{C} 51 \mathrm{~B}-\mathrm{C} 52 \mathrm{~B}$ & $117.6(3)$ \\
\hline $\mathrm{C} 56 \mathrm{~B}-\mathrm{C} 51 \mathrm{~B}-\mathrm{C} 25 \mathrm{~B}$ & $119.8(3)$ \\
\hline $\mathrm{C} 52 \mathrm{~B}-\mathrm{C} 51 \mathrm{~B}-\mathrm{C} 25 \mathrm{~B}$ & $122.6(3)$ \\
\hline $\mathrm{C} 56 \mathrm{~A}-\mathrm{C} 51 \mathrm{~A}-\mathrm{C} 52 \mathrm{~A}$ & $116.8(3)$ \\
\hline $\mathrm{C} 56 \mathrm{~A}-\mathrm{C} 51 \mathrm{~A}-\mathrm{C} 25 \mathrm{~A}$ & $122.2(3)$ \\
\hline $\mathrm{C} 52 \mathrm{~A}-\mathrm{C} 51 \mathrm{~A}-\mathrm{C} 25 \mathrm{~A}$ & $121.0(3)$ \\
\hline $\mathrm{C} 53 \mathrm{~B}-\mathrm{C} 52 \mathrm{~B}-\mathrm{C} 51 \mathrm{~B}$ & $121.1(3)$ \\
\hline $\mathrm{C} 53 \mathrm{~B}-\mathrm{C} 52 \mathrm{~B}-\mathrm{H} 52 \mathrm{~B}$ & 119.4 \\
\hline $\mathrm{C} 51 \mathrm{~B}-\mathrm{C} 52 \mathrm{~B}-\mathrm{H} 52 \mathrm{~B}$ & 119.4 \\
\hline $\mathrm{C} 51 \mathrm{~A}-\mathrm{C} 52 \mathrm{~A}-\mathrm{C} 53 \mathrm{~A}$ & $122.3(4)$ \\
\hline $\mathrm{C} 51 \mathrm{~A}-\mathrm{C} 52 \mathrm{~A}-\mathrm{H} 52 \mathrm{~A}$ & 118.9 \\
\hline $\mathrm{C} 53 \mathrm{~A}-\mathrm{C} 52 \mathrm{~A}-\mathrm{H} 52 \mathrm{~A}$ & 118.9 \\
\hline $\mathrm{C} 52 \mathrm{~B}-\mathrm{C} 53 \mathrm{~B}-\mathrm{C} 54 \mathrm{~B}$ & $120.1(3)$ \\
\hline $\mathrm{C} 52 \mathrm{~B}-\mathrm{C} 53 \mathrm{~B}-\mathrm{H} 53 \mathrm{~B}$ & 119.9 \\
\hline $\mathrm{C} 54 \mathrm{~B}-\mathrm{C} 53 \mathrm{~B}-\mathrm{H} 53 \mathrm{~B}$ & 119.9 \\
\hline $\mathrm{C} 54 \mathrm{~A}-\mathrm{C} 53 \mathrm{~A}-\mathrm{C} 52 \mathrm{~A}$ & $119.8(4)$ \\
\hline $\mathrm{C} 54 \mathrm{~A}-\mathrm{C} 53 \mathrm{~A}-\mathrm{H} 53 \mathrm{~A}$ & 120.1 \\
\hline $\mathrm{C} 52 \mathrm{~A}-\mathrm{C} 53 \mathrm{~A}-\mathrm{H} 53 \mathrm{~A}$ & 120.1 \\
\hline $\mathrm{O} 3 \mathrm{~B}-\mathrm{C} 54 \mathrm{~B}-\mathrm{C} 53 \mathrm{~B}$ & $115.8(3)$ \\
\hline $\mathrm{O} 3 \mathrm{~B}-\mathrm{C} 54 \mathrm{~B}-\mathrm{C} 55 \mathrm{~B}$ & $124.0(3)$ \\
\hline $\mathrm{C} 53 \mathrm{~B}-\mathrm{C} 54 \mathrm{~B}-\mathrm{C} 55 \mathrm{~B}$ & $120.2(3)$ \\
\hline $\mathrm{O} 3 \mathrm{~A}-\mathrm{C} 54 \mathrm{~A}-\mathrm{C} 53 \mathrm{~A}$ & $124.5(4)$ \\
\hline $\mathrm{O} 3 \mathrm{~A}-\mathrm{C} 54 \mathrm{~A}-\mathrm{C} 55 \mathrm{~A}$ & $116.6(4)$ \\
\hline $\mathrm{C} 53 \mathrm{~A}-\mathrm{C} 54 \mathrm{~A}-\mathrm{C} 55 \mathrm{~A}$ & $118.9(3)$ \\
\hline $\mathrm{C} 54 \mathrm{~B}-\mathrm{C} 55 \mathrm{~B}-\mathrm{C} 56 \mathrm{~B}$ & $119.0(3)$ \\
\hline $\mathrm{C} 54 \mathrm{~B}-\mathrm{C} 55 \mathrm{~B}-\mathrm{H} 55 \mathrm{~B}$ & 120.5 \\
\hline $\mathrm{C} 56 \mathrm{~B}-\mathrm{C} 55 \mathrm{~B}-\mathrm{H} 55 \mathrm{~B}$ & 120.5 \\
\hline $\mathrm{C} 56 \mathrm{~A}-\mathrm{C} 55 \mathrm{~A}-\mathrm{C} 54 \mathrm{~A}$ & $120.8(4)$ \\
\hline $\mathrm{C} 56 \mathrm{~A}-\mathrm{C} 55 \mathrm{~A}-\mathrm{H} 55 \mathrm{~A}$ & 119.6 \\
\hline $\mathrm{C} 54 \mathrm{~A}-\mathrm{C} 55 \mathrm{~A}-\mathrm{H} 55 \mathrm{~A}$ & 119.6 \\
\hline $\mathrm{C} 51 \mathrm{~B}-\mathrm{C} 56 \mathrm{~B}-\mathrm{C} 55 \mathrm{~B}$ & $121.9(3)$ \\
\hline
\end{tabular}




\begin{tabular}{|c|c|}
\hline $\mathrm{C} 11 \mathrm{~B}-\mathrm{C} 16 \mathrm{~B}-\mathrm{H} 16 \mathrm{~B}$ & 119.3 \\
\hline $\mathrm{C} 15 \mathrm{~B}-\mathrm{C} 16 \mathrm{~B}-\mathrm{H} 16 \mathrm{~B}$ & 119.3 \\
\hline $\mathrm{C} 15 \mathrm{~A}-\mathrm{C} 16 \mathrm{~A}-\mathrm{C} 11 \mathrm{~A}$ & $121.4(3)$ \\
\hline $\mathrm{C} 15 \mathrm{~A}-\mathrm{C} 16 \mathrm{~A}-\mathrm{H} 16 \mathrm{~A}$ & 119.3 \\
\hline $\mathrm{C} 11 \mathrm{~A}-\mathrm{C} 16 \mathrm{~A}-\mathrm{H} 16 \mathrm{~A}$ & 119.3 \\
\hline $\mathrm{O} 1 \mathrm{~B}-\mathrm{C} 21 \mathrm{~B}-\mathrm{C} 22 \mathrm{~B}$ & $120.4(3)$ \\
\hline $\mathrm{O} 1 \mathrm{~B}-\mathrm{C} 21 \mathrm{~B}-\mathrm{C} 2 \mathrm{~B}$ & $122.3(3)$ \\
\hline $\mathrm{C} 22 \mathrm{~B}-\mathrm{C} 21 \mathrm{~B}-\mathrm{C} 2 \mathrm{~B}$ & $117.4(2)$ \\
\hline $\mathrm{O} 1 \mathrm{~A}-\mathrm{C} 21 \mathrm{~A}-\mathrm{C} 22 \mathrm{~A}$ & $119.3(3)$ \\
\hline $\mathrm{O} 1 \mathrm{~A}-\mathrm{C} 21 \mathrm{~A}-\mathrm{C} 2 \mathrm{~A}$ & $122.0(3)$ \\
\hline $\mathrm{C} 22 \mathrm{~A}-\mathrm{C} 21 \mathrm{~A}-\mathrm{C} 2 \mathrm{~A}$ & $118.7(2)$ \\
\hline $\mathrm{C} 23 \mathrm{~B}-\mathrm{C} 22 \mathrm{~B}-\mathrm{C} 26 \mathrm{~B}$ & $117.6(3)$ \\
\hline $\mathrm{C} 23 \mathrm{~B}-\mathrm{C} 22 \mathrm{~B}-\mathrm{C} 21 \mathrm{~B}$ & $119.9(3)$ \\
\hline $\mathrm{C} 26 \mathrm{~B}-\mathrm{C} 22 \mathrm{~B}-\mathrm{C} 21 \mathrm{~B}$ & $122.3(3)$ \\
\hline $\mathrm{C} 23 \mathrm{~A}-\mathrm{C} 22 \mathrm{~A}-\mathrm{C} 26 \mathrm{~A}$ & $118.1(3)$ \\
\hline $\mathrm{C} 23 \mathrm{~A}-\mathrm{C} 22 \mathrm{~A}-\mathrm{C} 21 \mathrm{~A}$ & $119.4(3)$ \\
\hline $\mathrm{C} 26 \mathrm{~A}-\mathrm{C} 22 \mathrm{~A}-\mathrm{C} 21 \mathrm{~A}$ & $122.5(3)$ \\
\hline $\mathrm{C} 24 \mathrm{~B}-\mathrm{C} 23 \mathrm{~B}-\mathrm{C} 22 \mathrm{~B}$ & $119.5(3)$ \\
\hline $\mathrm{C} 24 \mathrm{~B}-\mathrm{C} 23 \mathrm{~B}-\mathrm{H} 23 \mathrm{~B}$ & 120.3 \\
\hline $\mathrm{C} 22 \mathrm{~B}-\mathrm{C} 23 \mathrm{~B}-\mathrm{H} 23 \mathrm{~B}$ & 120.3 \\
\hline $\mathrm{C} 24 \mathrm{~A}-\mathrm{C} 23 \mathrm{~A}-\mathrm{C} 22 \mathrm{~A}$ & $119.8(3)$ \\
\hline $\mathrm{C} 24 \mathrm{~A}-\mathrm{C} 23 \mathrm{~A}-\mathrm{H} 23 \mathrm{~A}$ & 120.1 \\
\hline $\mathrm{C} 22 \mathrm{~A}-\mathrm{C} 23 \mathrm{~A}-\mathrm{H} 23 \mathrm{~A}$ & 120.1 \\
\hline $\mathrm{C} 23 \mathrm{~B}-\mathrm{C} 24 \mathrm{~B}-\mathrm{C} 25 \mathrm{~B}$ & $120.0(3)$ \\
\hline $\mathrm{C} 23 \mathrm{~B}-\mathrm{C} 24 \mathrm{~B}-\mathrm{H} 24 \mathrm{~B}$ & 120.0 \\
\hline $\mathrm{C} 25 \mathrm{~B}-\mathrm{C} 24 \mathrm{~B}-\mathrm{H} 24 \mathrm{~B}$ & 120.0 \\
\hline $\mathrm{C} 23 \mathrm{~A}-\mathrm{C} 24 \mathrm{~A}-\mathrm{C} 25 \mathrm{~A}$ & $119.0(3)$ \\
\hline $\mathrm{C} 23 \mathrm{~A}-\mathrm{C} 24 \mathrm{~A}-\mathrm{H} 24 \mathrm{~A}$ & 120.5 \\
\hline $\mathrm{C} 25 \mathrm{~A}-\mathrm{C} 24 \mathrm{~A}-\mathrm{H} 24 \mathrm{~A}$ & 120.5 \\
\hline $\mathrm{N} 2 \mathrm{~B}-\mathrm{C} 25 \mathrm{~B}-\mathrm{C} 24 \mathrm{~B}$ & $120.8(3)$ \\
\hline $\mathrm{N} 2 \mathrm{~B}-\mathrm{C} 25 \mathrm{~B}-\mathrm{C} 51 \mathrm{~B}$ & $116.3(3)$ \\
\hline $\mathrm{C} 24 \mathrm{~B}-\mathrm{C} 25 \mathrm{~B}-\mathrm{C} 51 \mathrm{~B}$ & $122.8(3)$ \\
\hline $\mathrm{N} 2 \mathrm{~A}-\mathrm{C} 25 \mathrm{~A}-\mathrm{C} 24 \mathrm{~A}$ & $122.6(3)$ \\
\hline $\mathrm{N} 2 \mathrm{~A}-\mathrm{C} 25 \mathrm{~A}-\mathrm{C} 51 \mathrm{~A}$ & $117.7(3)$ \\
\hline $\mathrm{C} 24 \mathrm{~A}-\mathrm{C} 25 \mathrm{~A}-\mathrm{C} 51 \mathrm{~A}$ & $119.7(3)$ \\
\hline $\mathrm{C} 11 \mathrm{~B}-\mathrm{C} 1 \mathrm{~B}-\mathrm{C} 2 \mathrm{~B}-\mathrm{C} 28 \mathrm{~B}$ & $9.1(4)$ \\
\hline $\mathrm{C} 4 \mathrm{~B}-\mathrm{C} 1 \mathrm{~B}-\mathrm{C} 2 \mathrm{~B}-\mathrm{C} 28 \mathrm{~B}$ & $-120.5(3)$ \\
\hline $\mathrm{C} 11 \mathrm{~B}-\mathrm{C} 1 \mathrm{~B}-\mathrm{C} 2 \mathrm{~B}-\mathrm{C} 21 \mathrm{~B}$ & $-110.4(3)$ \\
\hline $\mathrm{C} 4 \mathrm{~B}-\mathrm{C} 1 \mathrm{~B}-\mathrm{C} 2 \mathrm{~B}-\mathrm{C} 21 \mathrm{~B}$ & $120.1(3)$ \\
\hline $\mathrm{C} 11 \mathrm{~B}-\mathrm{C} 1 \mathrm{~B}-\mathrm{C} 2 \mathrm{~B}-\mathrm{C} 3 \mathrm{~B}$ & $133.7(2)$ \\
\hline $\mathrm{C} 4 \mathrm{~B}-\mathrm{C} 1 \mathrm{~B}-\mathrm{C} 2 \mathrm{~B}-\mathrm{C} 3 \mathrm{~B}$ & $4.1(3)$ \\
\hline $\mathrm{C} 11 \mathrm{~A}-\mathrm{C} 1 \mathrm{~A}-\mathrm{C} 2 \mathrm{~A}-\mathrm{C} 28 \mathrm{~A}$ & $-10.6(4)$ \\
\hline $\mathrm{C} 4 \mathrm{~A}-\mathrm{C} 1 \mathrm{~A}-\mathrm{C} 2 \mathrm{~A}-\mathrm{C} 28 \mathrm{~A}$ & $117.3(3)$ \\
\hline $\mathrm{C} 11 \mathrm{~A}-\mathrm{C} 1 \mathrm{~A}-\mathrm{C} 2 \mathrm{~A}-\mathrm{C} 21 \mathrm{~A}$ & $110.1(3)$ \\
\hline $\mathrm{C} 4 \mathrm{~A}-\mathrm{C} 1 \mathrm{~A}-\mathrm{C} 2 \mathrm{~A}-\mathrm{C} 21 \mathrm{~A}$ & $-122.0(2)$ \\
\hline $\mathrm{C} 11 \mathrm{~A}-\mathrm{C} 1 \mathrm{~A}-\mathrm{C} 2 \mathrm{~A}-\mathrm{C} 3 \mathrm{~A}$ & $-135.8(2)$ \\
\hline $\mathrm{C} 4 \mathrm{~A}-\mathrm{C} 1 \mathrm{~A}-\mathrm{C} 2 \mathrm{~A}-\mathrm{C} 3 \mathrm{~A}$ & $-7.9(3)$ \\
\hline
\end{tabular}

\begin{tabular}{|c|c|}
\hline $\mathrm{C} 51 \mathrm{~B}-\mathrm{C} 56 \mathrm{~B}-\mathrm{H} 56 \mathrm{~B}$ & 119.1 \\
\hline $\mathrm{C} 55 \mathrm{~B}-\mathrm{C} 56 \mathrm{~B}-\mathrm{H} 56 \mathrm{~B}$ & 119.1 \\
\hline $\mathrm{C} 55 \mathrm{~A}-\mathrm{C} 56 \mathrm{~A}-\mathrm{C} 51 \mathrm{~A}$ & $121.4(3)$ \\
\hline $\mathrm{C} 55 \mathrm{~A}-\mathrm{C} 56 \mathrm{~A}-\mathrm{H} 56 \mathrm{~A}$ & 119.3 \\
\hline $\mathrm{C} 51 \mathrm{~A}-\mathrm{C} 56 \mathrm{~A}-\mathrm{H} 56 \mathrm{~A}$ & 119.3 \\
\hline $\mathrm{C} 6 \mathrm{~B}-\mathrm{N} 1 \mathrm{~B}-\mathrm{C} 3 \mathrm{~B}$ & $120.8(2)$ \\
\hline $\mathrm{C} 6 \mathrm{~B}-\mathrm{N} 1 \mathrm{~B}-\mathrm{C} 4 \mathrm{~B}$ & $108.9(2)$ \\
\hline $\mathrm{C} 3 \mathrm{~B}-\mathrm{N} 1 \mathrm{~B}-\mathrm{C} 4 \mathrm{~B}$ & $108.6(2)$ \\
\hline $\mathrm{C} 6 \mathrm{~A}-\mathrm{N} 1 \mathrm{~A}-\mathrm{C} 4 \mathrm{~A}$ & $110.1(2)$ \\
\hline $\mathrm{C} 6 \mathrm{~A}-\mathrm{N} 1 \mathrm{~A}-\mathrm{C} 3 \mathrm{~A}$ & $120.4(2)$ \\
\hline $\mathrm{C} 4 \mathrm{~A}-\mathrm{N} 1 \mathrm{~A}-\mathrm{C} 3 \mathrm{~A}$ & $107.3(2)$ \\
\hline $\mathrm{C} 26 \mathrm{~B}-\mathrm{N} 2 \mathrm{~B}-\mathrm{C} 25 \mathrm{~B}$ & $119.1(3)$ \\
\hline $\mathrm{C} 25 \mathrm{~A}-\mathrm{N} 2 \mathrm{~A}-\mathrm{C} 26 \mathrm{~A}$ & $117.8(3)$ \\
\hline $\mathrm{C} 37 \mathrm{~B}-\mathrm{N} 3 \mathrm{~B}-\mathrm{C} 36 \mathrm{~B}$ & $110.7(3)$ \\
\hline $\mathrm{C} 37 \mathrm{~B}-\mathrm{N} 3 \mathrm{~B}-\mathrm{H} 3 \mathrm{~B}$ & $123(2)$ \\
\hline $\mathrm{C} 36 \mathrm{~B}-\mathrm{N} 3 \mathrm{~B}-\mathrm{H} 3 \mathrm{~B}$ & $127(2)$ \\
\hline $\mathrm{C} 37 \mathrm{~A}-\mathrm{N} 3 \mathrm{~A}-\mathrm{C} 36 \mathrm{~A}$ & $111.4(3)$ \\
\hline $\mathrm{C} 37 \mathrm{~A}-\mathrm{N} 3 \mathrm{~A}-\mathrm{H} 3 \mathrm{~A}$ & $119(3)$ \\
\hline $\mathrm{C} 36 \mathrm{~A}-\mathrm{N} 3 \mathrm{~A}-\mathrm{H} 3 \mathrm{~A}$ & $129(3)$ \\
\hline $\mathrm{C} 54 \mathrm{~B}-\mathrm{O} 3 \mathrm{~B}-\mathrm{C} 57 \mathrm{~B}$ & $117.7(3)$ \\
\hline $\mathrm{C} 54 \mathrm{~A}-\mathrm{O} 3 \mathrm{~A}-\mathrm{C} 57 \mathrm{~A}$ & $118.2(4)$ \\
\hline $\mathrm{C} 6 \mathrm{~B}-\mathrm{S} 1 \mathrm{~B}-\mathrm{C} 5 \mathrm{~B}$ & $93.18(14)$ \\
\hline $\mathrm{C} 6 \mathrm{~A}-\mathrm{S} 1 \mathrm{~A}-\mathrm{C} 5 \mathrm{~A}$ & $93.69(14)$ \\
\hline $\mathrm{O} 3 \mathrm{~B}-\mathrm{C} 57 \mathrm{~B}-\mathrm{H} 57 \mathrm{~A}$ & 109.5 \\
\hline $\mathrm{O} 3 \mathrm{~B}-\mathrm{C} 57 \mathrm{~B}-\mathrm{H} 57 \mathrm{C}$ & 109.5 \\
\hline $\mathrm{H} 57 \mathrm{~A}-\mathrm{C} 57 \mathrm{~B}-\mathrm{H} 57 \mathrm{C}$ & 109.5 \\
\hline $\mathrm{O} 3 \mathrm{~B}-\mathrm{C} 57 \mathrm{~B}-\mathrm{H} 57 \mathrm{~B}$ & 109.5 \\
\hline $\mathrm{H} 57 \mathrm{~A}-\mathrm{C} 57 \mathrm{~B}-\mathrm{H} 57 \mathrm{~B}$ & 109.5 \\
\hline $\mathrm{H} 57 \mathrm{C}-\mathrm{C} 57 \mathrm{~B}-\mathrm{H} 57 \mathrm{~B}$ & 109.5 \\
\hline $\mathrm{O} 3 \mathrm{~A}-\mathrm{C} 57 \mathrm{~A}-\mathrm{H} 57 \mathrm{D}$ & 109.5 \\
\hline $\mathrm{O} 3 \mathrm{~A}-\mathrm{C} 57 \mathrm{~A}-\mathrm{H} 57 \mathrm{E}$ & 109.5 \\
\hline $\mathrm{H} 57 \mathrm{D}-\mathrm{C} 57 \mathrm{~A}-\mathrm{H} 57 \mathrm{E}$ & 109.5 \\
\hline $\mathrm{O} 3 \mathrm{~A}-\mathrm{C} 57 \mathrm{~A}-\mathrm{H} 57 \mathrm{~F}$ & 109.5 \\
\hline $\mathrm{H} 57 \mathrm{D}-\mathrm{C} 57 \mathrm{~A}-\mathrm{H} 57 \mathrm{~F}$ & 109.5 \\
\hline $\mathrm{H} 57 \mathrm{E}-\mathrm{C} 57 \mathrm{~A}-\mathrm{H} 57 \mathrm{~F}$ & 109.5 \\
\hline $\mathrm{C} 37 \mathrm{~B}-\mathrm{C} 3 \mathrm{~B}-\mathrm{C} 31 \mathrm{~B}-\mathrm{C} 36 \mathrm{~B}$ & $1.6(3)$ \\
\hline $\mathrm{C} 2 \mathrm{~B}-\mathrm{C} 3 \mathrm{~B}-\mathrm{C} 31 \mathrm{~B}-\mathrm{C} 36 \mathrm{~B}$ & $124.8(3)$ \\
\hline $\mathrm{N} 1 \mathrm{~B}-\mathrm{C} 3 \mathrm{~B}-\mathrm{C} 31 \mathrm{~B}-\mathrm{C} 32 \mathrm{~B}$ & $54.8(4)$ \\
\hline $\mathrm{C} 37 \mathrm{~B}-\mathrm{C} 3 \mathrm{~B}-\mathrm{C} 31 \mathrm{~B}-\mathrm{C} 32 \mathrm{~B}$ & $174.4(3)$ \\
\hline $\mathrm{C} 2 \mathrm{~B}-\mathrm{C} 3 \mathrm{~B}-\mathrm{C} 31 \mathrm{~B}-\mathrm{C} 32 \mathrm{~B}$ & $-62.4(4)$ \\
\hline $\mathrm{N} 1 \mathrm{~A}-\mathrm{C} 3 \mathrm{~A}-\mathrm{C} 31 \mathrm{~A}-\mathrm{C} 32 \mathrm{~A}$ & $-51.8(4)$ \\
\hline $\mathrm{C} 37 \mathrm{~A}-\mathrm{C} 3 \mathrm{~A}-\mathrm{C} 31 \mathrm{~A}-\mathrm{C} 32 \mathrm{~A}$ & $-173.2(3)$ \\
\hline $\mathrm{C} 2 \mathrm{~A}-\mathrm{C} 3 \mathrm{~A}-\mathrm{C} 31 \mathrm{~A}-\mathrm{C} 32 \mathrm{~A}$ & $65.6(4)$ \\
\hline $\mathrm{N} 1 \mathrm{~A}-\mathrm{C} 3 \mathrm{~A}-\mathrm{C} 31 \mathrm{~A}-\mathrm{C} 36 \mathrm{~A}$ & $126.0(3)$ \\
\hline $\mathrm{C} 37 \mathrm{~A}-\mathrm{C} 3 \mathrm{~A}-\mathrm{C} 31 \mathrm{~A}-\mathrm{C} 36 \mathrm{~A}$ & $4.6(3)$ \\
\hline $\mathrm{C} 2 \mathrm{~A}-\mathrm{C} 3 \mathrm{~A}-\mathrm{C} 31 \mathrm{~A}-\mathrm{C} 36 \mathrm{~A}$ & $-116.6(3)$ \\
\hline $\mathrm{C} 36 \mathrm{~B}-\mathrm{C} 31 \mathrm{~B}-\mathrm{C} 32 \mathrm{~B}-\mathrm{C} 33 \mathrm{~B}$ & $-2.5(5)$ \\
\hline
\end{tabular}




\begin{tabular}{|c|c|}
\hline $\mathrm{C} 28 \mathrm{~B}-\mathrm{C} 2 \mathrm{~B}-\mathrm{C} 3 \mathrm{~B}-\mathrm{N} 1 \mathrm{~B}$ & $147.3(2)$ \\
\hline $\mathrm{C} 21 \mathrm{~B}-\mathrm{C} 2 \mathrm{~B}-\mathrm{C} 3 \mathrm{~B}-\mathrm{N} 1 \mathrm{~B}$ & $-94.3(2)$ \\
\hline $\mathrm{C} 1 \mathrm{~B}-\mathrm{C} 2 \mathrm{~B}-\mathrm{C} 3 \mathrm{~B}-\mathrm{N} 1 \mathrm{~B}$ & $21.8(3)$ \\
\hline $\mathrm{C} 28 \mathrm{~B}-\mathrm{C} 2 \mathrm{~B}-\mathrm{C} 3 \mathrm{~B}-\mathrm{C} 31 \mathrm{~B}$ & $-90.5(3)$ \\
\hline $\mathrm{C} 21 \mathrm{~B}-\mathrm{C} 2 \mathrm{~B}-\mathrm{C} 3 \mathrm{~B}-\mathrm{C} 31 \mathrm{~B}$ & $28.0(3)$ \\
\hline $\mathrm{C} 1 \mathrm{~B}-\mathrm{C} 2 \mathrm{~B}-\mathrm{C} 3 \mathrm{~B}-\mathrm{C} 31 \mathrm{~B}$ & $144.0(3)$ \\
\hline $\mathrm{C} 28 \mathrm{~B}-\mathrm{C} 2 \mathrm{~B}-\mathrm{C} 3 \mathrm{~B}-\mathrm{C} 37 \mathrm{~B}$ & $28.3(3)$ \\
\hline $\mathrm{C} 21 \mathrm{~B}-\mathrm{C} 2 \mathrm{~B}-\mathrm{C} 3 \mathrm{~B}-\mathrm{C} 37 \mathrm{~B}$ & $146.7(2)$ \\
\hline $\mathrm{C} 1 \mathrm{~B}-\mathrm{C} 2 \mathrm{~B}-\mathrm{C} 3 \mathrm{~B}-\mathrm{C} 37 \mathrm{~B}$ & $-97.3(3)$ \\
\hline $\mathrm{C} 28 \mathrm{~A}-\mathrm{C} 2 \mathrm{~A}-\mathrm{C} 3 \mathrm{~A}-\mathrm{N} 1 \mathrm{~A}$ & $-145.2(2)$ \\
\hline $\mathrm{C} 21 \mathrm{~A}-\mathrm{C} 2 \mathrm{~A}-\mathrm{C} 3 \mathrm{~A}-\mathrm{N} 1 \mathrm{~A}$ & $96.1(2)$ \\
\hline $\mathrm{C} 1 \mathrm{~A}-\mathrm{C} 2 \mathrm{~A}-\mathrm{C} 3 \mathrm{~A}-\mathrm{N} 1 \mathrm{~A}$ & $-19.7(3)$ \\
\hline $\mathrm{C} 28 \mathrm{~A}-\mathrm{C} 2 \mathrm{~A}-\mathrm{C} 3 \mathrm{~A}-\mathrm{C} 31 \mathrm{~A}$ & $91.5(3)$ \\
\hline $\mathrm{C} 21 \mathrm{~A}-\mathrm{C} 2 \mathrm{~A}-\mathrm{C} 3 \mathrm{~A}-\mathrm{C} 31 \mathrm{~A}$ & $-27.2(3)$ \\
\hline $\mathrm{C} 1 \mathrm{~A}-\mathrm{C} 2 \mathrm{~A}-\mathrm{C} 3 \mathrm{~A}-\mathrm{C} 31 \mathrm{~A}$ & $-143.1(2)$ \\
\hline $\mathrm{C} 28 \mathrm{~A}-\mathrm{C} 2 \mathrm{~A}-\mathrm{C} 3 \mathrm{~A}-\mathrm{C} 37 \mathrm{~A}$ & $-24.7(3)$ \\
\hline $\mathrm{C} 21 \mathrm{~A}-\mathrm{C} 2 \mathrm{~A}-\mathrm{C} 3 \mathrm{~A}-\mathrm{C} 37 \mathrm{~A}$ & $-143.5(2)$ \\
\hline $\mathrm{C} 1 \mathrm{~A}-\mathrm{C} 2 \mathrm{~A}-\mathrm{C} 3 \mathrm{~A}-\mathrm{C} 37 \mathrm{~A}$ & $100.7(2)$ \\
\hline $\mathrm{C} 11 \mathrm{~B}-\mathrm{C} 1 \mathrm{~B}-\mathrm{C} 4 \mathrm{~B}-\mathrm{N} 1 \mathrm{~B}$ & $-157.4(2)$ \\
\hline $\mathrm{C} 2 \mathrm{~B}-\mathrm{C} 1 \mathrm{~B}-\mathrm{C} 4 \mathrm{~B}-\mathrm{N} 1 \mathrm{~B}$ & $-28.5(3)$ \\
\hline $\mathrm{C} 11 \mathrm{~B}-\mathrm{C} 1 \mathrm{~B}-\mathrm{C} 4 \mathrm{~B}-\mathrm{C} 5 \mathrm{~B}$ & $89.0(3)$ \\
\hline $\mathrm{C} 2 \mathrm{~B}-\mathrm{C} 1 \mathrm{~B}-\mathrm{C} 4 \mathrm{~B}-\mathrm{C} 5 \mathrm{~B}$ & $-142.1(3)$ \\
\hline $\mathrm{C} 11 \mathrm{~A}-\mathrm{C} 1 \mathrm{~A}-\mathrm{C} 4 \mathrm{~A}-\mathrm{N} 1 \mathrm{~A}$ & $161.6(2)$ \\
\hline $\mathrm{C} 2 \mathrm{~A}-\mathrm{C} 1 \mathrm{~A}-\mathrm{C} 4 \mathrm{~A}-\mathrm{N} 1 \mathrm{~A}$ & $32.8(3)$ \\
\hline $\mathrm{C} 11 \mathrm{~A}-\mathrm{C} 1 \mathrm{~A}-\mathrm{C} 4 \mathrm{~A}-\mathrm{C} 5 \mathrm{~A}$ & $-83.7(3)$ \\
\hline $\mathrm{C} 2 \mathrm{~A}-\mathrm{C} 1 \mathrm{~A}-\mathrm{C} 4 \mathrm{~A}-\mathrm{C} 5 \mathrm{~A}$ & $147.5(3)$ \\
\hline $\mathrm{N} 1 \mathrm{~B}-\mathrm{C} 4 \mathrm{~B}-\mathrm{C} 5 \mathrm{~B}-\mathrm{S} 1 \mathrm{~B}$ & $38.7(3)$ \\
\hline $\mathrm{C} 1 \mathrm{~B}-\mathrm{C} 4 \mathrm{~B}-\mathrm{C} 5 \mathrm{~B}-\mathrm{S} 1 \mathrm{~B}$ & $150.9(2)$ \\
\hline $\mathrm{N} 1 \mathrm{~A}-\mathrm{C} 4 \mathrm{~A}-\mathrm{C} 5 \mathrm{~A}-\mathrm{S} 1 \mathrm{~A}$ & $-35.1(3)$ \\
\hline $\mathrm{C} 1 \mathrm{~A}-\mathrm{C} 4 \mathrm{~A}-\mathrm{C} 5 \mathrm{~A}-\mathrm{S} 1 \mathrm{~A}$ & $-147.7(2)$ \\
\hline $\mathrm{C} 4 \mathrm{~B}-\mathrm{C} 1 \mathrm{~B}-\mathrm{C} 11 \mathrm{~B}-\mathrm{C} 16 \mathrm{~B}$ & $-142.6(3)$ \\
\hline $\mathrm{C} 2 \mathrm{~B}-\mathrm{C} 1 \mathrm{~B}-\mathrm{C} 11 \mathrm{~B}-\mathrm{C} 16 \mathrm{~B}$ & $93.4(3)$ \\
\hline $\mathrm{C} 4 \mathrm{~B}-\mathrm{C} 1 \mathrm{~B}-\mathrm{C} 11 \mathrm{~B}-\mathrm{C} 12 \mathrm{~B}$ & $37.7(4)$ \\
\hline $\mathrm{C} 2 \mathrm{~B}-\mathrm{C} 1 \mathrm{~B}-\mathrm{C} 11 \mathrm{~B}-\mathrm{C} 12 \mathrm{~B}$ & $-86.3(4)$ \\
\hline $\mathrm{C} 4 \mathrm{~A}-\mathrm{C} 1 \mathrm{~A}-\mathrm{C} 11 \mathrm{~A}-\mathrm{C} 12 \mathrm{~A}$ & $-49.6(4)$ \\
\hline $\mathrm{C} 2 \mathrm{~A}-\mathrm{C} 1 \mathrm{~A}-\mathrm{C} 11 \mathrm{~A}-\mathrm{C} 12 \mathrm{~A}$ & $73.3(4)$ \\
\hline $\mathrm{C} 4 \mathrm{~A}-\mathrm{C} 1 \mathrm{~A}-\mathrm{C} 11 \mathrm{~A}-\mathrm{C} 16 \mathrm{~A}$ & $129.4(3)$ \\
\hline $\mathrm{C} 2 \mathrm{~A}-\mathrm{C} 1 \mathrm{~A}-\mathrm{C} 11 \mathrm{~A}-\mathrm{C} 16 \mathrm{~A}$ & $-107.7(3)$ \\
\hline $\mathrm{C} 16 \mathrm{~B}-\mathrm{C} 11 \mathrm{~B}-\mathrm{C} 12 \mathrm{~B}-\mathrm{C} 13 \mathrm{~B}$ & $-0.6(5)$ \\
\hline $\mathrm{C} 1 \mathrm{~B}-\mathrm{C} 11 \mathrm{~B}-\mathrm{C} 12 \mathrm{~B}-\mathrm{C} 13 \mathrm{~B}$ & $179.0(3)$ \\
\hline $\mathrm{C} 16 \mathrm{~A}-\mathrm{C} 11 \mathrm{~A}-\mathrm{C} 12 \mathrm{~A}-\mathrm{C} 13 \mathrm{~A}$ & $0.7(5)$ \\
\hline $\mathrm{C} 1 \mathrm{~A}-\mathrm{C} 11 \mathrm{~A}-\mathrm{C} 12 \mathrm{~A}-\mathrm{C} 13 \mathrm{~A}$ & $179.7(3)$ \\
\hline $\mathrm{C} 11 \mathrm{~B}-\mathrm{C} 12 \mathrm{~B}-\mathrm{C} 13 \mathrm{~B}-\mathrm{C} 14 \mathrm{~B}$ & $-0.7(5)$ \\
\hline $\mathrm{C} 11 \mathrm{~A}-\mathrm{C} 12 \mathrm{~A}-\mathrm{C} 13 \mathrm{~A}-\mathrm{C} 14 \mathrm{~A}$ & $-0.2(5)$ \\
\hline $\mathrm{C} 12 \mathrm{~B}-\mathrm{C} 13 \mathrm{~B}-\mathrm{C} 14 \mathrm{~B}-\mathrm{C} 15 \mathrm{~B}$ & $1.5(5)$ \\
\hline $\mathrm{C} 12 \mathrm{~B}-\mathrm{C} 13 \mathrm{~B}-\mathrm{C} 14 \mathrm{~B}-\mathrm{C} 11 \mathrm{~B}$ & $-179.2(3)$ \\
\hline $\mathrm{C} 12 \mathrm{~A}-\mathrm{C} 13 \mathrm{~A}-\mathrm{C} 14 \mathrm{~A}-\mathrm{C} 15 \mathrm{~A}$ & $0.3(5)$ \\
\hline $\mathrm{C} 12 \mathrm{~A}-\mathrm{C} 13 \mathrm{~A}-\mathrm{C} 14 \mathrm{~A}-\mathrm{C} 11 \mathrm{~A}$ & $179.1(2)$ \\
\hline
\end{tabular}

\begin{tabular}{|c|c|}
\hline $\mathrm{C} 3 \mathrm{~B}-\mathrm{C} 31 \mathrm{~B}-\mathrm{C} 32 \mathrm{~B}-\mathrm{C} 33 \mathrm{~B}$ & $-174.7(3)$ \\
\hline $\mathrm{C} 36 \mathrm{~A}-\mathrm{C} 31 \mathrm{~A}-\mathrm{C} 32 \mathrm{~A}-\mathrm{C} 33 \mathrm{~A}$ & $3.5(5)$ \\
\hline $\mathrm{C} 3 \mathrm{~A}-\mathrm{C} 31 \mathrm{~A}-\mathrm{C} 32 \mathrm{~A}-\mathrm{C} 33 \mathrm{~A}$ & $-178.9(3)$ \\
\hline $\mathrm{C} 31 \mathrm{~B}-\mathrm{C} 32 \mathrm{~B}-\mathrm{C} 33 \mathrm{~B}-\mathrm{C} 34 \mathrm{~B}$ & $1.0(6)$ \\
\hline $\mathrm{C} 31 \mathrm{~A}-\mathrm{C} 32 \mathrm{~A}-\mathrm{C} 33 \mathrm{~A}-\mathrm{C} 34 \mathrm{~A}$ & $-0.7(6)$ \\
\hline $\mathrm{C} 32 \mathrm{~B}-\mathrm{C} 33 \mathrm{~B}-\mathrm{C} 34 \mathrm{~B}-\mathrm{C} 35 \mathrm{~B}$ & $0.8(6)$ \\
\hline $\mathrm{C} 32 \mathrm{~A}-\mathrm{C} 33 \mathrm{~A}-\mathrm{C} 34 \mathrm{~A}-\mathrm{C} 35 \mathrm{~A}$ & $-1.8(7)$ \\
\hline $\mathrm{C} 33 \mathrm{~B}-\mathrm{C} 34 \mathrm{~B}-\mathrm{C} 35 \mathrm{~B}-\mathrm{C} 36 \mathrm{~B}$ & $-1.0(6)$ \\
\hline $\mathrm{C} 33 \mathrm{~A}-\mathrm{C} 34 \mathrm{~A}-\mathrm{C} 35 \mathrm{~A}-\mathrm{C} 36 \mathrm{~A}$ & $1.3(6)$ \\
\hline $\mathrm{C} 32 \mathrm{~B}-\mathrm{C} 31 \mathrm{~B}-\mathrm{C} 36 \mathrm{~B}-\mathrm{C} 35 \mathrm{~B}$ & $2.3(5)$ \\
\hline $\mathrm{C} 3 \mathrm{~B}-\mathrm{C} 31 \mathrm{~B}-\mathrm{C} 36 \mathrm{~B}-\mathrm{C} 35 \mathrm{~B}$ & $176.1(3)$ \\
\hline $\mathrm{C} 32 \mathrm{~B}-\mathrm{C} 31 \mathrm{~B}-\mathrm{C} 36 \mathrm{~B}-\mathrm{N} 3 \mathrm{~B}$ & $-175.2(3)$ \\
\hline $\mathrm{C} 3 \mathrm{~B}-\mathrm{C} 31 \mathrm{~B}-\mathrm{C} 36 \mathrm{~B}-\mathrm{N} 3 \mathrm{~B}$ & $-1.4(4)$ \\
\hline $\mathrm{C} 34 \mathrm{~B}-\mathrm{C} 35 \mathrm{~B}-\mathrm{C} 36 \mathrm{~B}-\mathrm{C} 31 \mathrm{~B}$ & $-0.6(5)$ \\
\hline $\mathrm{C} 34 \mathrm{~B}-\mathrm{C} 35 \mathrm{~B}-\mathrm{C} 36 \mathrm{~B}-\mathrm{N} 3 \mathrm{~B}$ & $176.6(3)$ \\
\hline $\mathrm{C} 34 \mathrm{~A}-\mathrm{C} 35 \mathrm{~A}-\mathrm{C} 36 \mathrm{~A}-\mathrm{C} 31 \mathrm{~A}$ & $1.6(6)$ \\
\hline $\mathrm{C} 34 \mathrm{~A}-\mathrm{C} 35 \mathrm{~A}-\mathrm{C} 36 \mathrm{~A}-\mathrm{N} 3 \mathrm{~A}$ & $-177.2(4)$ \\
\hline $\mathrm{C} 32 \mathrm{~A}-\mathrm{C} 31 \mathrm{~A}-\mathrm{C} 36 \mathrm{~A}-\mathrm{C} 35 \mathrm{~A}$ & $-4.0(5)$ \\
\hline $\mathrm{C} 3 \mathrm{~A}-\mathrm{C} 31 \mathrm{~A}-\mathrm{C} 36 \mathrm{~A}-\mathrm{C} 35 \mathrm{~A}$ & $177.9(3)$ \\
\hline $\mathrm{C} 32 \mathrm{~A}-\mathrm{C} 31 \mathrm{~A}-\mathrm{C} 36 \mathrm{~A}-\mathrm{N} 3 \mathrm{~A}$ & $175.0(3)$ \\
\hline $\mathrm{C} 3 \mathrm{~A}-\mathrm{C} 31 \mathrm{~A}-\mathrm{C} 36 \mathrm{~A}-\mathrm{N} 3 \mathrm{~A}$ & $-3.1(4)$ \\
\hline $\mathrm{N} 1 \mathrm{~B}-\mathrm{C} 3 \mathrm{~B}-\mathrm{C} 37 \mathrm{~B}-\mathrm{O} 2 \mathrm{~B}$ & $-60.6(4)$ \\
\hline $\mathrm{C} 31 \mathrm{~B}-\mathrm{C} 3 \mathrm{~B}-\mathrm{C} 37 \mathrm{~B}-\mathrm{O} 2 \mathrm{~B}$ & $-179.1(3)$ \\
\hline $\mathrm{C} 2 \mathrm{~B}-\mathrm{C} 3 \mathrm{~B}-\mathrm{C} 37 \mathrm{~B}-\mathrm{O} 2 \mathrm{~B}$ & $51.0(4)$ \\
\hline $\mathrm{N} 1 \mathrm{~B}-\mathrm{C} 3 \mathrm{~B}-\mathrm{C} 37 \mathrm{~B}-\mathrm{N} 3 \mathrm{~B}$ & $117.2(3)$ \\
\hline $\mathrm{C} 31 \mathrm{~B}-\mathrm{C} 3 \mathrm{~B}-\mathrm{C} 37 \mathrm{~B}-\mathrm{N} 3 \mathrm{~B}$ & $-1.2(3)$ \\
\hline $\mathrm{C} 2 \mathrm{~B}-\mathrm{C} 3 \mathrm{~B}-\mathrm{C} 37 \mathrm{~B}-\mathrm{N} 3 \mathrm{~B}$ & $-131.2(3)$ \\
\hline $\mathrm{N} 1 \mathrm{~A}-\mathrm{C} 3 \mathrm{~A}-\mathrm{C} 37 \mathrm{~A}-\mathrm{O} 2 \mathrm{~A}$ & $52.9(4)$ \\
\hline $\mathrm{C} 31 \mathrm{~A}-\mathrm{C} 3 \mathrm{~A}-\mathrm{C} 37 \mathrm{~A}-\mathrm{O} 2 \mathrm{~A}$ & $173.9(3)$ \\
\hline $\mathrm{C} 2 \mathrm{~A}-\mathrm{C} 3 \mathrm{~A}-\mathrm{C} 37 \mathrm{~A}-\mathrm{O} 2 \mathrm{~A}$ & $-60.0(4)$ \\
\hline $\mathrm{N} 1 \mathrm{~A}-\mathrm{C} 3 \mathrm{~A}-\mathrm{C} 37 \mathrm{~A}-\mathrm{N} 3 \mathrm{~A}$ & $-125.8(3)$ \\
\hline $\mathrm{C} 31 \mathrm{~A}-\mathrm{C} 3 \mathrm{~A}-\mathrm{C} 37 \mathrm{~A}-\mathrm{N} 3 \mathrm{~A}$ & $-4.7(3)$ \\
\hline $\mathrm{C} 2 \mathrm{~A}-\mathrm{C} 3 \mathrm{~A}-\mathrm{C} 37 \mathrm{~A}-\mathrm{N} 3 \mathrm{~A}$ & $121.3(3)$ \\
\hline $\mathrm{N} 2 \mathrm{~B}-\mathrm{C} 25 \mathrm{~B}-\mathrm{C} 51 \mathrm{~B}-\mathrm{C} 56 \mathrm{~B}$ & $-6.5(5)$ \\
\hline $\mathrm{C} 24 \mathrm{~B}-\mathrm{C} 25 \mathrm{~B}-\mathrm{C} 51 \mathrm{~B}-\mathrm{C} 56 \mathrm{~B}$ & $173.3(3)$ \\
\hline $\mathrm{N} 2 \mathrm{~B}-\mathrm{C} 25 \mathrm{~B}-\mathrm{C} 51 \mathrm{~B}-\mathrm{C} 52 \mathrm{~B}$ & $173.2(3)$ \\
\hline $\mathrm{C} 24 \mathrm{~B}-\mathrm{C} 25 \mathrm{~B}-\mathrm{C} 51 \mathrm{~B}-\mathrm{C} 52 \mathrm{~B}$ & $-7.0(5)$ \\
\hline $\mathrm{N} 2 \mathrm{~A}-\mathrm{C} 25 \mathrm{~A}-\mathrm{C} 51 \mathrm{~A}-\mathrm{C} 56 \mathrm{~A}$ & $37.5(5)$ \\
\hline $\mathrm{C} 24 \mathrm{~A}-\mathrm{C} 25 \mathrm{~A}-\mathrm{C} 51 \mathrm{~A}-\mathrm{C} 56 \mathrm{~A}$ & $-142.6(4)$ \\
\hline $\mathrm{N} 2 \mathrm{~A}-\mathrm{C} 25 \mathrm{~A}-\mathrm{C} 51 \mathrm{~A}-\mathrm{C} 52 \mathrm{~A}$ & $-143.9(4)$ \\
\hline $\mathrm{C} 24 \mathrm{~A}-\mathrm{C} 25 \mathrm{~A}-\mathrm{C} 51 \mathrm{~A}-\mathrm{C} 52 \mathrm{~A}$ & $36.0(5)$ \\
\hline $\mathrm{C} 56 \mathrm{~B}-\mathrm{C} 51 \mathrm{~B}-\mathrm{C} 52 \mathrm{~B}-\mathrm{C} 53 \mathrm{~B}$ & $-0.3(5)$ \\
\hline $\mathrm{C} 25 \mathrm{~B}-\mathrm{C} 51 \mathrm{~B}-\mathrm{C} 52 \mathrm{~B}-\mathrm{C} 53 \mathrm{~B}$ & $180.0(3)$ \\
\hline $\mathrm{C} 56 \mathrm{~A}-\mathrm{C} 51 \mathrm{~A}-\mathrm{C} 52 \mathrm{~A}-\mathrm{C} 53 \mathrm{~A}$ & $-0.9(6)$ \\
\hline $\mathrm{C} 25 \mathrm{~A}-\mathrm{C} 51 \mathrm{~A}-\mathrm{C} 52 \mathrm{~A}-\mathrm{C} 53 \mathrm{~A}$ & $-179.6(4)$ \\
\hline $\mathrm{C} 51 \mathrm{~B}-\mathrm{C} 52 \mathrm{~B}-\mathrm{C} 53 \mathrm{~B}-\mathrm{C} 54 \mathrm{~B}$ & $0.9(6)$ \\
\hline $\mathrm{C} 51 \mathrm{~A}-\mathrm{C} 52 \mathrm{~A}-\mathrm{C} 53 \mathrm{~A}-\mathrm{C} 54 \mathrm{~A}$ & $-0.1(7)$ \\
\hline $\mathrm{C} 52 \mathrm{~B}-\mathrm{C} 53 \mathrm{~B}-\mathrm{C} 54 \mathrm{~B}-\mathrm{O} 3 \mathrm{~B}$ & $179.5(3)$ \\
\hline
\end{tabular}




\begin{tabular}{|c|c|c|c|}
\hline $\mathrm{C} 13 \mathrm{~B}-\mathrm{C} 14 \mathrm{~B}-\mathrm{C} 15 \mathrm{~B}-\mathrm{C} 16 \mathrm{~B}$ & $-0.8(5)$ & $\mathrm{C} 52 \mathrm{~B}-\mathrm{C} 53 \mathrm{~B}-\mathrm{C} 54 \mathrm{~B}-\mathrm{C} 55 \mathrm{~B}$ & $-1.5(5)$ \\
\hline $\mathrm{C} 11 \mathrm{~B}-\mathrm{C} 14 \mathrm{~B}-\mathrm{C} 15 \mathrm{~B}-\mathrm{C} 16 \mathrm{~B}$ & $179.8(3)$ & $\mathrm{C} 52 \mathrm{~A}-\mathrm{C} 53 \mathrm{~A}-\mathrm{C} 54 \mathrm{~A}-\mathrm{O} 3 \mathrm{~A}$ & $179.5(4)$ \\
\hline $\mathrm{C} 13 \mathrm{~A}-\mathrm{C} 14 \mathrm{~A}-\mathrm{C} 15 \mathrm{~A}-\mathrm{C} 16 \mathrm{~A}$ & $-0.9(5)$ & $\mathrm{C} 52 \mathrm{~A}-\mathrm{C} 53 \mathrm{~A}-\mathrm{C} 54 \mathrm{~A}-\mathrm{C} 55 \mathrm{~A}$ & $1.4(7)$ \\
\hline $\mathrm{C} 11 \mathrm{~A}-\mathrm{C} 14 \mathrm{~A}-\mathrm{C} 15 \mathrm{~A}-\mathrm{C} 16 \mathrm{~A}$ & $-179.7(2)$ & $\mathrm{O} 3 \mathrm{~B}-\mathrm{C} 54 \mathrm{~B}-\mathrm{C} 55 \mathrm{~B}-\mathrm{C} 56 \mathrm{~B}$ & $-179.6(3)$ \\
\hline $\mathrm{C} 12 \mathrm{~B}-\mathrm{C} 11 \mathrm{~B}-\mathrm{C} 16 \mathrm{~B}-\mathrm{C} 15 \mathrm{~B}$ & $1.3(5)$ & $\mathrm{C} 53 \mathrm{~B}-\mathrm{C} 54 \mathrm{~B}-\mathrm{C} 55 \mathrm{~B}-\mathrm{C} 56 \mathrm{~B}$ & $1.5(5)$ \\
\hline $\mathrm{C} 1 \mathrm{~B}-\mathrm{C} 11 \mathrm{~B}-\mathrm{C} 16 \mathrm{~B}-\mathrm{C} 15 \mathrm{~B}$ & $-178.4(3)$ & $\mathrm{O} 3 \mathrm{~A}-\mathrm{C} 54 \mathrm{~A}-\mathrm{C} 55 \mathrm{~A}-\mathrm{C} 56 \mathrm{~A}$ & $179.9(4)$ \\
\hline $\mathrm{C} 14 \mathrm{~B}-\mathrm{C} 15 \mathrm{~B}-\mathrm{C} 16 \mathrm{~B}-\mathrm{C} 11 \mathrm{~B}$ & $-0.6(5)$ & $\mathrm{C} 53 \mathrm{~A}-\mathrm{C} 54 \mathrm{~A}-\mathrm{C} 55 \mathrm{~A}-\mathrm{C} 56 \mathrm{~A}$ & $-1.8(6)$ \\
\hline $\mathrm{C} 14 \mathrm{~A}-\mathrm{C} 15 \mathrm{~A}-\mathrm{C} 16 \mathrm{~A}-\mathrm{C} 11 \mathrm{~A}$ & $1.4(5)$ & $\mathrm{C} 52 \mathrm{~B}-\mathrm{C} 51 \mathrm{~B}-\mathrm{C} 56 \mathrm{~B}-\mathrm{C} 55 \mathrm{~B}$ & $0.3(5)$ \\
\hline $\mathrm{C} 12 \mathrm{~A}-\mathrm{C} 11 \mathrm{~A}-\mathrm{C} 16 \mathrm{~A}-\mathrm{C} 15 \mathrm{~A}$ & $-1.3(4)$ & $\mathrm{C} 25 \mathrm{~B}-\mathrm{C} 51 \mathrm{~B}-\mathrm{C} 56 \mathrm{~B}-\mathrm{C} 55 \mathrm{~B}$ & $-180.0(3)$ \\
\hline $\mathrm{C} 1 \mathrm{~A}-\mathrm{C} 11 \mathrm{~A}-\mathrm{C} 16 \mathrm{~A}-\mathrm{C} 15 \mathrm{~A}$ & $179.6(3)$ & $\mathrm{C} 54 \mathrm{~B}-\mathrm{C} 55 \mathrm{~B}-\mathrm{C} 56 \mathrm{~B}-\mathrm{C} 51 \mathrm{~B}$ & $-0.9(5)$ \\
\hline $\mathrm{C} 28 \mathrm{~B}-\mathrm{C} 2 \mathrm{~B}-\mathrm{C} 21 \mathrm{~B}-\mathrm{O} 1 \mathrm{~B}$ & $-141.2(3)$ & $\mathrm{C} 54 \mathrm{~A}-\mathrm{C} 55 \mathrm{~A}-\mathrm{C} 56 \mathrm{~A}-\mathrm{C} 51 \mathrm{~A}$ & $0.8(6)$ \\
\hline $\mathrm{C} 1 \mathrm{~B}-\mathrm{C} 2 \mathrm{~B}-\mathrm{C} 21 \mathrm{~B}-\mathrm{O} 1 \mathrm{~B}$ & $-16.5(4)$ & $\mathrm{C} 52 \mathrm{~A}-\mathrm{C} 51 \mathrm{~A}-\mathrm{C} 56 \mathrm{~A}-\mathrm{C} 55 \mathrm{~A}$ & $0.5(6)$ \\
\hline $\mathrm{C} 3 \mathrm{~B}-\mathrm{C} 2 \mathrm{~B}-\mathrm{C} 21 \mathrm{~B}-\mathrm{O} 1 \mathrm{~B}$ & $95.9(3)$ & $\mathrm{C} 25 \mathrm{~A}-\mathrm{C} 51 \mathrm{~A}-\mathrm{C} 56 \mathrm{~A}-\mathrm{C} 55 \mathrm{~A}$ & $179.2(3)$ \\
\hline $\mathrm{C} 28 \mathrm{~B}-\mathrm{C} 2 \mathrm{~B}-\mathrm{C} 21 \mathrm{~B}-\mathrm{C} 22 \mathrm{~B}$ & $38.4(3)$ & $\mathrm{S} 1 \mathrm{~B}-\mathrm{C} 6 \mathrm{~B}-\mathrm{N} 1 \mathrm{~B}-\mathrm{C} 3 \mathrm{~B}$ & $170.4(2)$ \\
\hline $\mathrm{C} 1 \mathrm{~B}-\mathrm{C} 2 \mathrm{~B}-\mathrm{C} 21 \mathrm{~B}-\mathrm{C} 22 \mathrm{~B}$ & $163.1(2)$ & $\mathrm{S} 1 \mathrm{~B}-\mathrm{C} 6 \mathrm{~B}-\mathrm{N} 1 \mathrm{~B}-\mathrm{C} 4 \mathrm{~B}$ & $43.7(3)$ \\
\hline $\mathrm{C} 3 \mathrm{~B}-\mathrm{C} 2 \mathrm{~B}-\mathrm{C} 21 \mathrm{~B}-\mathrm{C} 22 \mathrm{~B}$ & $-84.5(3)$ & $\mathrm{C} 31 \mathrm{~B}-\mathrm{C} 3 \mathrm{~B}-\mathrm{N} 1 \mathrm{~B}-\mathrm{C} 6 \mathrm{~B}$ & $60.9(3)$ \\
\hline $\mathrm{C} 28 \mathrm{~A}-\mathrm{C} 2 \mathrm{~A}-\mathrm{C} 21 \mathrm{~A}-\mathrm{O} 1 \mathrm{~A}$ & $147.6(3)$ & $\mathrm{C} 37 \mathrm{~B}-\mathrm{C} 3 \mathrm{~B}-\mathrm{N} 1 \mathrm{~B}-\mathrm{C} 6 \mathrm{~B}$ & $-52.0(3)$ \\
\hline $\mathrm{C} 1 \mathrm{~A}-\mathrm{C} 2 \mathrm{~A}-\mathrm{C} 21 \mathrm{~A}-\mathrm{O} 1 \mathrm{~A}$ & $22.3(4)$ & $\mathrm{C} 2 \mathrm{~B}-\mathrm{C} 3 \mathrm{~B}-\mathrm{N} 1 \mathrm{~B}-\mathrm{C} 6 \mathrm{~B}$ & $-169.9(2)$ \\
\hline $\mathrm{C} 3 \mathrm{~A}-\mathrm{C} 2 \mathrm{~A}-\mathrm{C} 21 \mathrm{~A}-\mathrm{O} 1 \mathrm{~A}$ & $-89.0(3)$ & $\mathrm{C} 31 \mathrm{~B}-\mathrm{C} 3 \mathrm{~B}-\mathrm{N} 1 \mathrm{~B}-\mathrm{C} 4 \mathrm{~B}$ & $-172.2(2)$ \\
\hline $\mathrm{C} 28 \mathrm{~A}-\mathrm{C} 2 \mathrm{~A}-\mathrm{C} 21 \mathrm{~A}-\mathrm{C} 22 \mathrm{~A}$ & $-35.4(3)$ & $\mathrm{C} 37 \mathrm{~B}-\mathrm{C} 3 \mathrm{~B}-\mathrm{N} 1 \mathrm{~B}-\mathrm{C} 4 \mathrm{~B}$ & $74.9(3)$ \\
\hline $\mathrm{C} 1 \mathrm{~A}-\mathrm{C} 2 \mathrm{~A}-\mathrm{C} 21 \mathrm{~A}-\mathrm{C} 22 \mathrm{~A}$ & $-160.7(2)$ & $\mathrm{C} 2 \mathrm{~B}-\mathrm{C} 3 \mathrm{~B}-\mathrm{N} 1 \mathrm{~B}-\mathrm{C} 4 \mathrm{~B}$ & $-43.0(3)$ \\
\hline $\mathrm{C} 3 \mathrm{~A}-\mathrm{C} 2 \mathrm{~A}-\mathrm{C} 21 \mathrm{~A}-\mathrm{C} 22 \mathrm{~A}$ & $88.0(3)$ & $\mathrm{C} 5 \mathrm{~B}-\mathrm{C} 4 \mathrm{~B}-\mathrm{N} 1 \mathrm{~B}-\mathrm{C} 6 \mathrm{~B}$ & $-55.4(3)$ \\
\hline $\mathrm{O} 1 \mathrm{~B}-\mathrm{C} 21 \mathrm{~B}-\mathrm{C} 22 \mathrm{~B}-\mathrm{C} 23 \mathrm{~B}$ & $-11.0(5)$ & $\mathrm{C} 1 \mathrm{~B}-\mathrm{C} 4 \mathrm{~B}-\mathrm{N} 1 \mathrm{~B}-\mathrm{C} 6 \mathrm{~B}$ & $179.8(2)$ \\
\hline $\mathrm{C} 2 \mathrm{~B}-\mathrm{C} 21 \mathrm{~B}-\mathrm{C} 22 \mathrm{~B}-\mathrm{C} 23 \mathrm{~B}$ & $169.4(3)$ & $\mathrm{C} 5 \mathrm{~B}-\mathrm{C} 4 \mathrm{~B}-\mathrm{N} 1 \mathrm{~B}-\mathrm{C} 3 \mathrm{~B}$ & $171.2(2)$ \\
\hline $\mathrm{O} 1 \mathrm{~B}-\mathrm{C} 21 \mathrm{~B}-\mathrm{C} 22 \mathrm{~B}-\mathrm{C} 26 \mathrm{~B}$ & $173.7(3)$ & $\mathrm{C} 1 \mathrm{~B}-\mathrm{C} 4 \mathrm{~B}-\mathrm{N} 1 \mathrm{~B}-\mathrm{C} 3 \mathrm{~B}$ & $46.4(3)$ \\
\hline $\mathrm{C} 2 \mathrm{~B}-\mathrm{C} 21 \mathrm{~B}-\mathrm{C} 22 \mathrm{~B}-\mathrm{C} 26 \mathrm{~B}$ & $-6.0(4)$ & $\mathrm{S} 1 \mathrm{~A}-\mathrm{C} 6 \mathrm{~A}-\mathrm{N} 1 \mathrm{~A}-\mathrm{C} 4 \mathrm{~A}$ & $-43.0(3)$ \\
\hline $\mathrm{O} 1 \mathrm{~A}-\mathrm{C} 21 \mathrm{~A}-\mathrm{C} 22 \mathrm{~A}-\mathrm{C} 23 \mathrm{~A}$ & $8.0(5)$ & $\mathrm{S} 1 \mathrm{~A}-\mathrm{C} 6 \mathrm{~A}-\mathrm{N} 1 \mathrm{~A}-\mathrm{C} 3 \mathrm{~A}$ & $-168.6(2)$ \\
\hline $\mathrm{C} 2 \mathrm{~A}-\mathrm{C} 21 \mathrm{~A}-\mathrm{C} 22 \mathrm{~A}-\mathrm{C} 23 \mathrm{~A}$ & $-169.1(3)$ & $\mathrm{C} 5 \mathrm{~A}-\mathrm{C} 4 \mathrm{~A}-\mathrm{N} 1 \mathrm{~A}-\mathrm{C} 6 \mathrm{~A}$ & $52.7(3)$ \\
\hline $\mathrm{O} 1 \mathrm{~A}-\mathrm{C} 21 \mathrm{~A}-\mathrm{C} 22 \mathrm{~A}-\mathrm{C} 26 \mathrm{~A}$ & $-172.8(3)$ & $\mathrm{C} 1 \mathrm{~A}-\mathrm{C} 4 \mathrm{~A}-\mathrm{N} 1 \mathrm{~A}-\mathrm{C} 6 \mathrm{~A}$ & $178.1(2)$ \\
\hline $\mathrm{C} 2 \mathrm{~A}-\mathrm{C} 21 \mathrm{~A}-\mathrm{C} 22 \mathrm{~A}-\mathrm{C} 26 \mathrm{~A}$ & $10.2(4)$ & $\mathrm{C} 5 \mathrm{~A}-\mathrm{C} 4 \mathrm{~A}-\mathrm{N} 1 \mathrm{~A}-\mathrm{C} 3 \mathrm{~A}$ & $-174.6(2)$ \\
\hline $\mathrm{C} 26 \mathrm{~B}-\mathrm{C} 22 \mathrm{~B}-\mathrm{C} 23 \mathrm{~B}-\mathrm{C} 24 \mathrm{~B}$ & $2.1(5)$ & $\mathrm{C} 1 \mathrm{~A}-\mathrm{C} 4 \mathrm{~A}-\mathrm{N} 1 \mathrm{~A}-\mathrm{C} 3 \mathrm{~A}$ & $-49.2(3)$ \\
\hline $\mathrm{C} 21 \mathrm{~B}-\mathrm{C} 22 \mathrm{~B}-\mathrm{C} 23 \mathrm{~B}-\mathrm{C} 24 \mathrm{~B}$ & $-173.5(3)$ & $\mathrm{C} 31 \mathrm{~A}-\mathrm{C} 3 \mathrm{~A}-\mathrm{N} 1 \mathrm{~A}-\mathrm{C} 6 \mathrm{~A}$ & $-63.0(3)$ \\
\hline $\mathrm{C} 26 \mathrm{~A}-\mathrm{C} 22 \mathrm{~A}-\mathrm{C} 23 \mathrm{~A}-\mathrm{C} 24 \mathrm{~A}$ & $-1.0(5)$ & $\mathrm{C} 37 \mathrm{~A}-\mathrm{C} 3 \mathrm{~A}-\mathrm{N} 1 \mathrm{~A}-\mathrm{C} 6 \mathrm{~A}$ & $51.4(4)$ \\
\hline $\mathrm{C} 21 \mathrm{~A}-\mathrm{C} 22 \mathrm{~A}-\mathrm{C} 23 \mathrm{~A}-\mathrm{C} 24 \mathrm{~A}$ & $178.2(3)$ & $\mathrm{C} 2 \mathrm{~A}-\mathrm{C} 3 \mathrm{~A}-\mathrm{N} 1 \mathrm{~A}-\mathrm{C} 6 \mathrm{~A}$ & $170.1(3)$ \\
\hline $\mathrm{C} 22 \mathrm{~B}-\mathrm{C} 23 \mathrm{~B}-\mathrm{C} 24 \mathrm{~B}-\mathrm{C} 25 \mathrm{~B}$ & $1.1(5)$ & $\mathrm{C} 31 \mathrm{~A}-\mathrm{C} 3 \mathrm{~A}-\mathrm{N} 1 \mathrm{~A}-\mathrm{C} 4 \mathrm{~A}$ & $170.1(2)$ \\
\hline $\mathrm{C} 22 \mathrm{~A}-\mathrm{C} 23 \mathrm{~A}-\mathrm{C} 24 \mathrm{~A}-\mathrm{C} 25 \mathrm{~A}$ & $0.9(5)$ & $\mathrm{C} 37 \mathrm{~A}-\mathrm{C} 3 \mathrm{~A}-\mathrm{N} 1 \mathrm{~A}-\mathrm{C} 4 \mathrm{~A}$ & $-75.5(3)$ \\
\hline $\mathrm{C} 23 \mathrm{~B}-\mathrm{C} 24 \mathrm{~B}-\mathrm{C} 25 \mathrm{~B}-\mathrm{N} 2 \mathrm{~B}$ & $-3.3(5)$ & $\mathrm{C} 2 \mathrm{~A}-\mathrm{C} 3 \mathrm{~A}-\mathrm{N} 1 \mathrm{~A}-\mathrm{C} 4 \mathrm{~A}$ & $43.3(3)$ \\
\hline $\mathrm{C} 23 \mathrm{~B}-\mathrm{C} 24 \mathrm{~B}-\mathrm{C} 25 \mathrm{~B}-\mathrm{C} 51 \mathrm{~B}$ & $176.9(3)$ & $\mathrm{C} 22 \mathrm{~B}-\mathrm{C} 26 \mathrm{~B}-\mathrm{N} 2 \mathrm{~B}-\mathrm{C} 25 \mathrm{~B}$ & $1.2(5)$ \\
\hline $\mathrm{C} 23 \mathrm{~A}-\mathrm{C} 24 \mathrm{~A}-\mathrm{C} 25 \mathrm{~A}-\mathrm{N} 2 \mathrm{~A}$ & $-0.2(5)$ & $\mathrm{C} 27 \mathrm{~B}-\mathrm{C} 26 \mathrm{~B}-\mathrm{N} 2 \mathrm{~B}-\mathrm{C} 25 \mathrm{~B}$ & $-178.7(3)$ \\
\hline $\mathrm{C} 23 \mathrm{~A}-\mathrm{C} 24 \mathrm{~A}-\mathrm{C} 25 \mathrm{~A}-\mathrm{C} 51 \mathrm{~A}$ & $179.9(3)$ & $\mathrm{C} 24 \mathrm{~B}-\mathrm{C} 25 \mathrm{~B}-\mathrm{N} 2 \mathrm{~B}-\mathrm{C} 26 \mathrm{~B}$ & $2.2(5)$ \\
\hline $\mathrm{C} 23 \mathrm{~B}-\mathrm{C} 22 \mathrm{~B}-\mathrm{C} 26 \mathrm{~B}-\mathrm{N} 2 \mathrm{~B}$ & $-3.3(5)$ & $\mathrm{C} 51 \mathrm{~B}-\mathrm{C} 25 \mathrm{~B}-\mathrm{N} 2 \mathrm{~B}-\mathrm{C} 26 \mathrm{~B}$ & $-178.0(3)$ \\
\hline $\mathrm{C} 21 \mathrm{~B}-\mathrm{C} 22 \mathrm{~B}-\mathrm{C} 26 \mathrm{~B}-\mathrm{N} 2 \mathrm{~B}$ & $172.1(3)$ & $\mathrm{C} 24 \mathrm{~A}-\mathrm{C} 25 \mathrm{~A}-\mathrm{N} 2 \mathrm{~A}-\mathrm{C} 26 \mathrm{~A}$ & $-0.4(5)$ \\
\hline $\mathrm{C} 23 \mathrm{~B}-\mathrm{C} 22 \mathrm{~B}-\mathrm{C} 26 \mathrm{~B}-\mathrm{C} 27 \mathrm{~B}$ & $176.5(3)$ & $\mathrm{C} 51 \mathrm{~A}-\mathrm{C} 25 \mathrm{~A}-\mathrm{N} 2 \mathrm{~A}-\mathrm{C} 26 \mathrm{~A}$ & $179.5(3)$ \\
\hline $\mathrm{C} 21 \mathrm{~B}-\mathrm{C} 22 \mathrm{~B}-\mathrm{C} 26 \mathrm{~B}-\mathrm{C} 27 \mathrm{~B}$ & $-8.0(5)$ & $\mathrm{C} 22 \mathrm{~A}-\mathrm{C} 26 \mathrm{~A}-\mathrm{N} 2 \mathrm{~A}-\mathrm{C} 25 \mathrm{~A}$ & $0.2(4)$ \\
\hline $\mathrm{C} 23 \mathrm{~A}-\mathrm{C} 22 \mathrm{~A}-\mathrm{C} 26 \mathrm{~A}-\mathrm{N} 2 \mathrm{~A}$ & $0.5(4)$ & $\mathrm{C} 27 \mathrm{~A}-\mathrm{C} 26 \mathrm{~A}-\mathrm{N} 2 \mathrm{~A}-\mathrm{C} 25 \mathrm{~A}$ & $-178.0(3)$ \\
\hline $\mathrm{C} 21 \mathrm{~A}-\mathrm{C} 22 \mathrm{~A}-\mathrm{C} 26 \mathrm{~A}-\mathrm{N} 2 \mathrm{~A}$ & $-178.8(3)$ & $\mathrm{O} 2 \mathrm{~B}-\mathrm{C} 37 \mathrm{~B}-\mathrm{N} 3 \mathrm{~B}-\mathrm{C} 36 \mathrm{~B}$ & $178.3(3)$ \\
\hline $\mathrm{C} 23 \mathrm{~A}-\mathrm{C} 22 \mathrm{~A}-\mathrm{C} 26 \mathrm{~A}-\mathrm{C} 27 \mathrm{~A}$ & $178.6(3)$ & $\mathrm{C} 3 \mathrm{~B}-\mathrm{C} 37 \mathrm{~B}-\mathrm{N} 3 \mathrm{~B}-\mathrm{C} 36 \mathrm{~B}$ & $0.5(3)$ \\
\hline $\mathrm{C} 21 \mathrm{~A}-\mathrm{C} 22 \mathrm{~A}-\mathrm{C} 26 \mathrm{~A}-\mathrm{C} 27 \mathrm{~A}$ & $-0.6(4)$ & $\mathrm{C} 31 \mathrm{~B}-\mathrm{C} 36 \mathrm{~B}-\mathrm{N} 3 \mathrm{~B}-\mathrm{C} 37 \mathrm{~B}$ & $0.6(4)$ \\
\hline
\end{tabular}




$\begin{array}{llll}\mathrm{N} 2 \mathrm{~B}-\mathrm{C} 26 \mathrm{~B}-\mathrm{C} 27 \mathrm{~B}-\mathrm{C} 28 \mathrm{~B} & 166.4(3) & \mathrm{C} 35 \mathrm{~B}-\mathrm{C} 36 \mathrm{~B}-\mathrm{N} 3 \mathrm{~B}-\mathrm{C} 37 \mathrm{~B} & -176.8(3) \\ \mathrm{C} 22 \mathrm{~B}-\mathrm{C} 26 \mathrm{~B}-\mathrm{C} 27 \mathrm{~B}-\mathrm{C} 28 \mathrm{~B} & -13.5(4) & \mathrm{O} 2 \mathrm{~A}-\mathrm{C} 37 \mathrm{~A}-\mathrm{N} 3 \mathrm{~A}-\mathrm{C} 36 \mathrm{~A} & -175.5(3) \\ \mathrm{N} 2 \mathrm{~A}-\mathrm{C} 26 \mathrm{~A}-\mathrm{C} 27 \mathrm{~A}-\mathrm{C} 28 \mathrm{~A} & -163.0(3) & \mathrm{C} 3 \mathrm{~A}-\mathrm{C} 37 \mathrm{~A}-\mathrm{N} 3 \mathrm{~A}-\mathrm{C} 36 \mathrm{~A} & 3.2(3) \\ \mathrm{C} 22 \mathrm{~A}-\mathrm{C} 26 \mathrm{~A}-\mathrm{C} 27 \mathrm{~A}-\mathrm{C} 28 \mathrm{~A} & 18.8(4) & \mathrm{C} 35 \mathrm{~A}-\mathrm{C} 36 \mathrm{~A}-\mathrm{N} 3 \mathrm{~A}-\mathrm{C} 37 \mathrm{~A} & 178.8(4) \\ \mathrm{C} 26 \mathrm{~B}-\mathrm{C} 27 \mathrm{~B}-\mathrm{C} 28 \mathrm{~B}-\mathrm{C} 2 \mathrm{~B} & 49.4(4) & \mathrm{C} 31 \mathrm{~A}-\mathrm{C} 36 \mathrm{~A}-\mathrm{N} 3 \mathrm{~A}-\mathrm{C} 37 \mathrm{~A} & -0.2(4) \\ \mathrm{C} 21 \mathrm{~B}-\mathrm{C} 2 \mathrm{~B}-\mathrm{C} 28 \mathrm{~B}-\mathrm{C} 27 \mathrm{~B} & -60.4(3) & \mathrm{C} 53 \mathrm{~B}-\mathrm{C} 54 \mathrm{~B}-\mathrm{O} 3 \mathrm{~B}-\mathrm{C} 57 \mathrm{~B} & -175.7(3) \\ \mathrm{C} 1 \mathrm{~B}-\mathrm{C} 2 \mathrm{~B}-\mathrm{C} 28 \mathrm{~B}-\mathrm{C} 27 \mathrm{~B} & 178.6(2) & \mathrm{C} 55 \mathrm{~B}-\mathrm{C} 54 \mathrm{~B}-\mathrm{O} 3 \mathrm{~B}-\mathrm{C} 57 \mathrm{~B} & 5.3(5) \\ \mathrm{C} 3 \mathrm{~B}-\mathrm{C} 2 \mathrm{~B}-\mathrm{C} 28 \mathrm{~B}-\mathrm{C} 27 \mathrm{~B} & 59.5(3) & \mathrm{C} 53 \mathrm{~A}-\mathrm{C} 54 \mathrm{~A}-\mathrm{O} 3 \mathrm{~A}-\mathrm{C} 57 \mathrm{~A} & -1.5(7) \\ \mathrm{C} 21 \mathrm{~A}-\mathrm{C} 2 \mathrm{~A}-\mathrm{C} 28 \mathrm{~A}-\mathrm{C} 27 \mathrm{~A} & 54.7(3) & \mathrm{C} 55 \mathrm{~A}-\mathrm{C} 54 \mathrm{~A}-\mathrm{O} 3 \mathrm{~A}-\mathrm{C} 57 \mathrm{~A} & 176.7(4) \\ \mathrm{C} 1 \mathrm{~A}-\mathrm{C} 2 \mathrm{~A}-\mathrm{C} 28 \mathrm{~A}-\mathrm{C} 27 \mathrm{~A} & 176.9(2) & \mathrm{N} 1 \mathrm{~B}-\mathrm{C} 6 \mathrm{~B}-\mathrm{S} 1 \mathrm{~B}-\mathrm{C} 5 \mathrm{~B} & -16.2(2) \\ \mathrm{C} 3 \mathrm{~A}-\mathrm{C} 2 \mathrm{~A}-\mathrm{C} 28 \mathrm{~A}-\mathrm{C} 27 \mathrm{~A} & -64.2(3) & \mathrm{C} 4 \mathrm{~B}-\mathrm{C} 5 \mathrm{~B}-\mathrm{S} 1 \mathrm{~B}-\mathrm{C} 6 \mathrm{~B} & -13.1(2) \\ \mathrm{C} 26 \mathrm{~A}-\mathrm{C} 27 \mathrm{~A}-\mathrm{C} 28 \mathrm{~A}-\mathrm{C} 2 \mathrm{~A} & -48.1(4) & \mathrm{N} 1 \mathrm{~A}-\mathrm{C} 6 \mathrm{~A}-\mathrm{S} 1 \mathrm{~A}-\mathrm{C} 5 \mathrm{~A} & 17.3(2) \\ \mathrm{N} 1 \mathrm{~B}-\mathrm{C} 3 \mathrm{~B}-\mathrm{C} 31 \mathrm{~B}-\mathrm{C} 36 \mathrm{~B} & -118.0(3) & \mathrm{C} 4 \mathrm{~A}-\mathrm{C} 5 \mathrm{~A}-\mathrm{S} 1 \mathrm{~A}-\mathrm{C} 6 \mathrm{~A} & 10.3(2)\end{array}$

Hydrogen-bond geometry $\left(\AA,{ }^{\circ}\right)$

$C g 8$ and $C g 17$ are the centroids of the $\mathrm{C} 51 A-\mathrm{C} 56 A$ and $\mathrm{C} 11 B-\mathrm{C} 16 B$ rings, respectively.

\begin{tabular}{lllll}
\hline$D-\mathrm{H} \cdots A$ & $D-\mathrm{H}$ & $\mathrm{H} \cdots A$ & $D \cdots A$ & $D-\mathrm{H} \cdots A$ \\
\hline $\mathrm{N} 3 A-\mathrm{H} 3 A \cdots \mathrm{O} 2 A^{\mathrm{i}}$ & $0.83(4)$ & $2.05(4)$ & $2.874(4)$ & $172(4)$ \\
$\mathrm{N} 3 B-\mathrm{H} 3 B \cdots \mathrm{N} 2 A^{\mathrm{ii}}$ & $0.91(4)$ & $2.24(4)$ & $3.136(4)$ & $169(3)$ \\
$\mathrm{C} 23 B-\mathrm{H} 23 B \cdots \mathrm{O} 1 A$ & 0.93 & 2.36 & $3.066(4)$ & 132 \\
$\mathrm{C} 27 A-\mathrm{H} 27 D \cdots \mathrm{O} 2 B^{\mathrm{iii}}$ & 0.97 & 2.40 & $3.371(4)$ & 177 \\
$\mathrm{C} 28 A-\mathrm{H} 28 C \cdots \mathrm{O} 3 B^{\mathrm{iv}}$ & 0.97 & 2.55 & $3.517(4)$ & 175 \\
$\mathrm{C} 57 B-\mathrm{H} 57 C \cdots \mathrm{O} 2 B^{\mathrm{v}}$ & 0.96 & 2.50 & $3.349(5)$ & 147 \\
$\mathrm{C} 5 A-\mathrm{H} 5 A 1 \cdots C g 17^{\mathrm{vi}}$ & 0.97 & 2.91 & $3.711(4)$ & 140 \\
$\mathrm{C} 27 B-\mathrm{H} 27 A \cdots C g 8^{\mathrm{iv}}$ & 0.97 & 2.89 & $3.783(4)$ & 154 \\
\hline
\end{tabular}

Symmetry codes: (i) $-x,-y+1,-z+1$; (ii) $x, y-1, z$; (iii) $x, y+1, z$; (iv) $-x+1,-y+1,-z$; (v) $-x+1,-y,-z$; (vi) $-x+1,-y,-z+1$. 